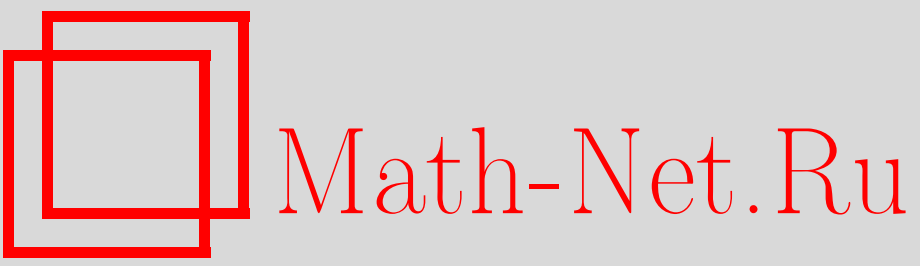

Н. Г. Чебочко, Деформации классических алгебр Ли с однородной системой корней в характеристике два. I, Матем. сб., 2005, том 196, номер 9, 125-156

DOI: https://doi.org/10.4213/sm1423

Использование Общероссийского математического портала Math-Net.Ru подразумевает, что вы прочитали и согласны с пользовательским соглашением

http://www.mathnet.ru/rus/agreement

Параметры загрузки:

IP : 3.82 .47 .9

26 апреля 2023 г., 10:55:44 


\author{
Н.Г. Чебочко
}

\title{
Деформации классических алгебр Ли с однородной системой корней в характеристике два. I
}

\begin{abstract}
В работе исследуются пространства локальных деформаций классических алгебр Ли с однородной системой корней над полем $K$ характеристики 2 . Под классической алгеброй Ли над полем $K$ мы понимаем алгебру Ли простой алгебраической групшы либо ее факторалгебру по центру. Описание деформаций алгебр Ли представляет интерес в связи с классификацией простых алгебр Ли.

Библиография: 13 названий.
\end{abstract}

\section{§1. Введение}

Над полем характеристики 2 и 3 классические алгебры Ли не определяются однозначно своей системой корней. В работе [1] построены параметрические семейства неизоморфных простых алгебр Ли характеристики 3 , которые являются глобальными деформациями алгебры Ли $C_{2}$. Согласно [2] алгебра Ли $C_{2}-$ единственная среди алгебр Ли серий $A_{n}, B_{n}, C_{n}, D_{n}$, допускающая нетривиальные деформации при $p=3$. Полное описание глобальных деформаций алгебры Ли $C_{2}$ получено в работе [3]. В работе [4] установлено, что над полем характеристики $p>3$ все классические алгебры Ли являются жесткими. В работах [5] и [6] предложена новая схема исследования жесткости и доказана жесткость классических алгебр Ли всех типов над полем характеристики $p>2$, кроме алгебры Ли типа $C_{2}$ при $p=3$. При $p=2$ некоторые деформации алгебры Ли типа $G_{2}$ были построены в [7].

Достаточным условием отсутствия деформаций является тривиальность второй группы когомологий алгебры Ли с коэффициентами в присоединенном модуле (пространства локальных деформаций). В настоящей работе найдены пространства локальных деформаций классических алгебр Ли с однородной системой корней над полем характеристики 2. При этом используются методы, разработанные в [5].

Алгебры Ли типа $A_{l}, l+1 \equiv 0(2), D_{l}, E_{7}$ имеют центр. О соответствуюших факторалгебрах будем говорить, что они имеют тип $\bar{A}_{l}, \bar{D}_{l}, \bar{E}_{7}$.

В работе доказана

ТеОрема. Пусть $L$ - алгебра Ли над полем характеристики 2. Тогда

(1) если $L$ имеет один из типов $A_{l}, l>1, D_{l}, l \equiv 1(2), E_{6}, E_{7}, E_{8}, \bar{A}_{l}$, $l \neq 3,5, \bar{D}_{l}, l \equiv 0(2), l \neq 6$, mo $H^{2}(L, L)=0$;

(2) если $L$ имеет mun $\bar{A}_{l}, l=3,5$, mo $\operatorname{dim} H^{2}(L, L)=20$;

Работа выполнена при поддержке Российского фонда фундаментальных исследований (грант № 02-01-00725). 
(3) если $L$ имеeт mun $D_{4}$, mo $\operatorname{dim} H^{2}(L, L)=24$;

(4) если $L$ uмеeт mun $D_{l}, l \equiv 0(2), l>4, \bar{D}_{l}, l \equiv 1(2), \operatorname{modim} H^{2}(L, L)=2 l$;

(5) если $L$ uмeет mun $\bar{D}_{6}$, mo $\operatorname{dim} H^{2}(L, L)=64$;

(6) если $L$ имеет тuп $\bar{E}_{7}$, mo $\operatorname{dim} H^{2}(L, L)=56$.

В ходе доказательства теоремы найдены базисы второй группы когомологий. Также найдено естественное описание $H^{2}(L, L)$ как модуля над Aut $L$ для алгебр Ли типа $\bar{D}_{l}, l \equiv 1(2)$, и $\bar{A}_{3}$ (теоремы 1 и 2 ).

Обозначим через $L$ алгебру Ли одного из типов $A_{l}, l>1, D_{l}, E_{6}, E_{7}, E_{8}$ или ее факторалгебру по центру над алгебраически замкнутым полем $K$ характеристики 2 , через $R$ обозначим систему корней соответствуюшей комплексной классической алгебры Ли.

Группа Aut $L$ содержит присоединенную группу Шевалле $G(L)$ (см. [8]). Основной комплекс разлагаем в прямую сумму весовых подкомплексов, используя естественное действие максимального тора группы $G(L)$. Соответствующие группы когомологий являются весовыми подпространствами в групше когомологий основного комплекса.

Доказательство теоремы состоит из двух частей. В первой части (§3) доказывается тривиальность группы $H_{0}^{2}(L, L)$. Здесь используются методы и результаты работы [5]. В пп. 3.1 и 3.2 рассматриваются случаи, когда $L$ не имеет центра и когда $L$ имеет центр соответственно.

Во второй части $(\S 4)$ вычисляются группы $H_{\mu}^{2}(L, L), \mu \neq 0$. Обозначим через $\mathscr{H}$ подалгебру Картана в $L$. Так как $L$ действует тривиально на $H^{2}(L, L)$, то веса $H^{2}(L, L)$ содержатся в множестве $\Lambda_{2}(R)=\{\mu=\alpha+\beta+\gamma \mid \alpha, \beta, \gamma \in$ $R \cup\{0\}, \mu(h)=0 \forall h \in \mathscr{H}\}$. В п. 4.1 доказываем, что $\Lambda_{2}(R) \subset M_{1} \cup M_{2} \cup M_{3} \cup M_{4}$, где

$$
\begin{aligned}
& M_{1}=\{2 \delta, \delta \in R \cup\{0\}\}, \\
& M_{2}=\{\alpha+\beta+\gamma \mid \alpha, \beta, \gamma \in R,\langle\alpha, \beta\rangle=\langle\alpha, \gamma\rangle=\langle\beta, \gamma\rangle=1\}, \\
& M_{3}=\{\alpha+\beta \mid \alpha, \beta \in R,\langle\alpha, \beta\rangle=0\}, \\
& M_{4}=\{\alpha+\beta+\gamma \mid \alpha, \beta, \gamma \in R,\langle\alpha, \beta\rangle=\langle\alpha, \gamma\rangle=\langle\beta, \gamma\rangle=0\}
\end{aligned}
$$

При этом веса могут содержаться в $M_{2}$ или в $M_{3}$, только если система $R$ имеет тип $D_{l}$ или $A_{3}$, в $M_{4}$, только если $R$ имеет тип $A_{5}, D_{6}$ или $E_{7}$. Несложные рассуждения показывают, что $H_{\mu}^{2}(L, L)=0$ для любого $\mu \in M_{1}$. Далее доказываем, что ненулевые когомологии веса из $M_{2}$ имеет только алгебра Ли типа $\bar{A}_{3}$, ненулевые когомологии веса из $M_{3}$ имеют только алгебры Ли типа $D_{l}, l \equiv 0(2), \bar{D}_{l}, l \equiv 1(2)$, или $\bar{A}_{3}$, а ненулевые когомологии веса из $M_{4}$ имеют только алгебры Ли типа $\bar{A}_{5}$, $\bar{D}_{6}$ или $\bar{E}_{7}$.

В $\S 2$ собраны вспомогательные утверждения о классических алгебрах Ли с однородными системами корней.

Автор выражает благодарность М.И. Кузнецову за постоянное внимание к работе и полезные замечания. Ему принадлежит доказательство предложения 2 о тривиальности $H^{*}(L, L)$ для алгебры Ли типа $A_{2}$, основанное на использовании аналога элемента Казимира при $p=2$. Отметим, что подобный элемент существует и для некоторых других типов алгебр Ли с однородной системой корней. 


\section{§2. Вспомогательные утверждения}

Мы рассматриваем только однородные системы корней (все корни имеют одинаковую длину), т.е. системы типа $A_{l}, D_{l}, E_{6}, E_{7}, E_{8}$.

Пусть $R$ - система корней. Через $\langle\alpha, \beta\rangle$ для $\alpha, \beta \in R$ будем обозначать числа Картана. В однородных системах корней $\langle\alpha, \beta\rangle=\langle\beta, \alpha\rangle$ для любых $\alpha, \beta \in R$.

Из [9; гл. VI, $§ 1$, предложение 8 и теорема 1] следует

Лемма 1. Пусть $R$-однородная система корней, $\alpha, \beta \in R$. Тогда

(1) $\alpha+\beta \in R \Leftrightarrow\langle\alpha, \beta\rangle=-1 ; \alpha-\beta \in R \Leftrightarrow\langle\alpha, \beta\rangle=1$;

(2) $\alpha=\beta \Leftrightarrow\langle\alpha, \beta\rangle=2 ; \alpha=-\beta \Leftrightarrow\langle\alpha, \beta\rangle=-2$;

(3) если $\alpha \neq \pm \beta$, mо $\alpha+\beta \notin R$ u $\alpha-\beta \notin R \Leftrightarrow\langle\alpha, \beta\rangle=0$.

В однородных системах корней длины серий корней не превышают 1 и все корни сопряжены относительно действия группы Вейля $W(R)$.

Пусть $L_{\mathbb{C}}$ - комплексная простая алгебра Ли, $\mathscr{H}_{\mathbb{C}}-$ картановская подалгебра, $R$ - система корней $L_{\mathbb{C}}, B=\left\{\alpha_{1}, \ldots, \alpha_{l}\right\}$ - базис $R$. В $L_{\mathbb{C}}$ выберем базис Шевалле $\left\{h_{1}, \ldots, h_{l}, e_{\gamma}, \gamma \in R\right\}$.

Пусть $L_{\mathbb{Z}}$ - форма Шевалле. Классическая алгебра Ли над полем $K$ характеристики 2 определяется как $L_{K}=L_{\mathbb{Z}} \otimes_{\mathbb{Z}} K$. Также определим алгебру Ли над простым подполем $\mathbb{F}_{2}: L_{\mathbb{F}_{2}}=L_{\mathbb{Z}} \otimes_{\mathbb{Z}} \mathbb{F}_{2}$. Базис Шевалле в $L_{\mathbb{C}}$ определяет базис в $L_{K}$. Его элементы будем обозначать по-прежнему $\left\{h_{1}, \ldots, h_{l}, e_{\gamma}, \gamma \in R\right\}$. Используя лемму 1 , получаем, что структурные уравнения в алгебрах Ли с однородной системой корней в этом базисе имеют следующий вид (см., например, [10]):

1) $\left[h_{i}, h_{j}\right]=0, i, j=\overline{1, l}$;

2) $\left[h_{i}, e_{\alpha}\right]=\left\langle\alpha, \alpha_{i}\right\rangle e_{\alpha}, i=\overline{1, l}, \alpha \in R$;

3) $\left[e_{\alpha}, e_{-\alpha}\right]=h_{\alpha}$-целочисленная линейная комбинация элементов $h_{i}, i=\overline{1, l}$;

4) если $\alpha, \beta, \alpha+\beta \in R$, то $\left[e_{\alpha}, e_{\beta}\right]=e_{\alpha+\beta}$;

5) если $\alpha, \beta \in R, \alpha+\beta \notin R, \alpha+\beta \neq 0$, то $\left[e_{\alpha}, e_{\beta}\right]=0$.

Так как $h_{\alpha}$ - единственный элемент из $\mathscr{H}_{\mathbb{C}}$ такой, что $\left(h_{\alpha}, h\right)=2 \alpha(h) /(\alpha, \alpha)$ для любого $h \in \mathscr{H}_{\mathbb{C}}$, то верна

ЛЕмма 2. Если $R$ - однородная система корней, $\alpha=x_{1} \alpha_{1}+\cdots+x_{l} \alpha_{l}-$ разлохсение $\alpha \in R$ по простым корням, то $h_{\alpha}=x_{1} h_{1}+\cdots+x_{l} h_{l}$.

СлЕДСТВИЕ. Если $\alpha, \beta, \alpha+\beta \in R$, mo $h_{\alpha+\beta}=h_{\alpha}+h_{\beta}$.

Всюду в дальнейшем через $L$ будем обозначать алгебру Ли одного из типов $A_{l}$, $l>1, D_{l}, E_{6}, E_{7}, E_{8}$ или ее факторалгебру по центру над алгебраически замкнутьм полем $K$ характеристики 2. Система корней $R$ будет в равной степени относиться как к самим алгебрам Ли, так и к их факторалгебрам по центру. Ранг системы $R$ будет называться рангом алгебры Ли $L$. Обозначим через $\mathscr{H}$ подалгебру, порожденную $\left\{h_{1}, \ldots, h_{l}\right\}$.

Система корней $R$ является системой векторов в вешественном евклидовом пространстве $V_{\mathbb{R}}$. Элементы из $R$ можно рассматривать как линейные функции на подалгебре $\mathscr{H}$ в $L$ (со значениями в $K)$. Отметим, что $\alpha\left(h_{\beta}\right)=\langle\alpha, \beta\rangle$. Однако числа Картана мы рассматриваем в кольце целых чисел, а значения функций на подалгебре Картана - в поле $K$. Поэтому будем писать, что $\langle\alpha, \beta\rangle \equiv 0(2)$, а $\alpha\left(h_{\beta}\right)=0$. 
ЛЕмма 3. Если $R$-однородная система ранга $l>1$, то любой корень из $R$ является ненулевой функцией на подалгебре $\mathscr{H}$.

ДокАЗАТЕЛЬСтво. Пусть $\alpha \in R$. Если $\left.\alpha\right|_{\mathscr{H}}=0$, то $\langle\alpha, \beta\rangle \equiv 0(2)$ для любого $\beta \in R$. Из леммы 1 следует, что $\langle\alpha, \beta\rangle=0, \pm 1$ при $\beta \neq \pm \alpha$. Таким образом, если $\alpha \in R$ является нулевой функцией на $\mathscr{H}$, то для любого $\beta \in R, \beta \neq \pm \alpha$, выполняется $\langle\alpha, \beta\rangle=0$. Это противоречит неприводимости системы $R$. Следовательно, $\alpha$ - ненулевая функция на $R$.

СлЕДСТВИЕ 1. Подалгебра $\mathscr{H}$ является подалгеброй Картана в L.

СЛЕДСТВИЕ 2. Для любого $\alpha \in R \quad h_{\alpha} \neq 0$ и не содержится в иентре алгебры Ли $L$.

Пусть $Q(R)$ - подгруппа в $V_{\mathbb{R}}$, порожденная корнями. Если $\mu=\sum \gamma_{i} \in Q(R)$, то через $\langle\mu, \alpha\rangle$ для $\alpha \in R$ будем обозначать целое число, равное $\sum\left\langle\gamma_{i}, \alpha\right\rangle$.

Лемма 4. Пусть $L$ - алгебра Ли одного из типов $A_{l}, D_{l}, E_{6}, E_{7}, E_{8}$, $\alpha, \beta, \gamma \in R$. Тогда

(1) ecлu $\alpha \neq \pm \beta$, mo $h_{\alpha}+h_{\beta} \neq 0$;

(2) если $\alpha+\beta+\gamma \notin 2 Q(R)$, mo $h_{\alpha}+h_{\beta}+h_{\gamma} \neq 0$.

ДокАЗАТЕльство. (1) Если $\alpha+\beta \in R$ или $\alpha-\beta \in R$, то $h_{\alpha}+h_{\beta}=h_{\alpha \pm \beta}$ и утверждение леммы следует из следствия 2 леммы 3 . Поэтому достаточно рассмотреть случай $\langle\alpha, \beta\rangle=0$ (см. лемму 1$)$.

Пусть $\alpha, \beta \in R,\langle\alpha, \beta\rangle=0$. Тогда система корней $S=\langle\alpha, \beta\rangle_{\mathbb{R}} \cap R$ имеет тип $A_{1} \times A_{1}$.

Согласно [9; гл. VI, $\S 1$, п. 7, предложение 24] существует базис системы $R$, содержащий базис системы $S$. Пусть $\left\{\delta_{1}, \ldots, \delta_{l}\right\}$ - такой базис, $\left\{\delta_{1}, \delta_{2}\right\}$ - базис системы $S$. Не ограничивая обшности, можем считать, что $\alpha= \pm \delta_{1}, \beta= \pm \delta_{2}$. Так как $W(R)$ действует просто транзитивно на множестве базисов $R[9 ;$ гл. VI, $\S 1$, п. 5, теорема 2], то сушествует $w \in W(R)$ такое, что $w(\alpha)=\alpha_{i}, w(\beta)=\alpha_{j}$ для некоторых $i \neq j$. Тогда предположение $h_{\alpha}+h_{\beta}=0$ приводит к $h_{i}+h_{j}=0$. Получаем противоречие тому, что $\left\{h_{1}, \ldots, h_{l}, e_{\gamma}, \gamma \in R\right\}-$ базис в $L$. Следовательно, $h_{\alpha}+h_{\beta} \neq 0$ ни для каких $\alpha \neq \pm \beta$.

(2) Если $\alpha= \pm \beta$, то $h_{\alpha}+h_{\beta}+h_{\gamma}=h_{\gamma}$ и утверждение следует из следствия 2 леммы 3. Аналогично, лемма верна, если $\alpha= \pm \gamma$ и $\beta= \pm \gamma$.

Если $\alpha+\beta \in R$ или $\alpha-\beta \in R$, то $h_{\alpha}+h_{\beta}+h_{\gamma}=h_{\alpha \pm \beta}+h_{\gamma}$ и утверждение леммы следует из п. (1). Аналогично, если $\alpha \pm \gamma$ или $\beta \pm \gamma$ являются корнями. Поэтому достаточно рассмотреть случай $\langle\alpha, \beta\rangle=\langle\alpha, \gamma\rangle=\langle\beta, \gamma\rangle=0$ (см. лемму 1 ).

Рассмотрим систему $S=\langle\alpha, \beta, \gamma\rangle_{\mathbb{R}} \cap R$. Из условия $\langle\alpha, \beta\rangle=\langle\alpha, \gamma\rangle=\langle\beta, \gamma\rangle=0$ следует, что $S$ имеет тип $A_{1} \times A_{1} \times A_{1}$. Так же, как при доказательстве п. (1), показываем, что существует $w \in W(R)$ такое, что $w(\alpha)=\alpha_{i}, w(\beta)=\alpha_{j}, w(\gamma)=\alpha_{k}$ для некоторых $i \neq j, i \neq k, j \neq k$. Так как $h_{i}+h_{j}+h_{k} \neq 0$, то $h_{\alpha}+h_{\beta}+h_{\gamma} \neq 0$.

Приведем в этом параграфе некоторые сведения о когомологиях алгебры Ли $L$.

Пусть $C^{\bullet}(L, L)$ - стандартный комплекс алгебры Ли $L$. Группа $\operatorname{Aut}(L)$ действует естественным образом на $C^{\bullet}(L, L)$. Основной комплекс можно разложить в прямую сумму весовых подкомплексов, используя действие максимального тора группы Шевалле $G(L)$. Соответствующие группы когомологий являются весовыми 
подпространствами в группе когомологий основного комплекса. Через $C_{\mu}^{k}(L, L)$, $Z_{\mu}^{k}(L, L), B_{\mu}^{k}(L, L), H_{\mu}^{k}(L, L)$ будем обозначать пространство коцепей, коциклов, кограниц и когомологий веса $\mu$ соответственно. Пространство $C^{k}(L, L)$ отождествляется с пространством $L^{*} \wedge \cdots \wedge L^{*} \otimes L$. Выбирая базис Шевалле в $L$, мы естественным образомполучаем базис в $C^{k}(L, L)$. Если $e_{\alpha_{1}}^{*} \wedge \cdots \wedge e_{\alpha_{k}}^{*} \otimes e_{\alpha_{k+1}} \in C_{\mu}^{k}(L, L)$, то $\mu(h)=\left(-\alpha_{1}-\cdots-\alpha_{k}+\alpha_{k+1}\right)(h), h \in \mathscr{H}$.

Любой элемент $\psi \in Z_{\mu}^{2}(L, L)$ можно представить в виде линейной комбинации элементов $x_{1}^{*} \wedge x_{2}^{*} \otimes x_{3}$, где $x_{i}$ - вектор базиса Шевалле, $i=1,2,3$, с коэффицциентами $\widehat{\psi}\left(x_{1}, x_{2} ; x_{3}\right)$. Если $x_{3}$ определяется по $\psi, x_{1}, x_{2}$, то вместо $\widehat{\psi}\left(x_{1}, x_{2} ; x_{3}\right)$ будем писать просто $\widehat{\psi}\left(x_{1}, x_{2}\right)$.

Так как $H^{2}\left(L_{K}, L_{K}\right)=H^{2}\left(L_{\mathbb{F}_{2}}, L_{\mathbb{F}_{2}}\right) \otimes_{\mathbb{F}_{2}} K$ (классическая алгебра Ли определена над $\mathbb{Z})$, то всюду в дальнейшем будем считать, что $\widehat{\psi}\left(x_{1}, x_{2} ; x_{3}\right)=0,1$.

Лемма 5. Пусть $\psi \in C_{\mu}^{2}(L, L), \mu(h)=0$ для всех $h \in \mathscr{H}$. Тогда для любых $\gamma, \delta \in R, h \in \mathscr{H}$ имеем $d \psi\left(e_{\gamma}, e_{\delta}, h\right)=\left[\psi\left(e_{\gamma}, h\right), e_{\delta}\right]+\left[\psi\left(e_{\delta}, h\right), e_{\gamma}\right]+\psi\left(\left[e_{\gamma}, e_{\delta}\right], h\right)$.

ДоказАТЕЛЬСтво. Очевидно, что $\left[\psi\left(e_{\gamma}, e_{\delta}\right), h\right]=(\gamma+\delta+\mu)(h) \psi\left(e_{\gamma}, e_{\delta}\right)$. Поэтому

$$
\begin{aligned}
& {\left[\psi\left(e_{\gamma}, e_{\delta}\right), h\right]+\psi\left(\left[e_{\gamma}, h\right], e_{\delta}\right)+\psi\left(\left[e_{\delta}, h\right], e_{\gamma}\right)} \\
& \quad=((\gamma+\delta+\mu)(h)+\gamma(h)+\delta(h)) \psi\left(e_{\gamma}, e_{\delta}\right) \\
& \quad=(\mu(h)+2 \gamma(h)+2 \delta(h)) \psi\left(e_{\gamma}, e_{\delta}\right)=0 .
\end{aligned}
$$

ЛЕмма 6. Если $\psi \in Z_{0}^{2}(L, L)$, mo $\psi\left(h, h^{\prime}\right)=0 u \widehat{\psi}\left(e_{\alpha}, h\right)=\widehat{\psi}\left(e_{-\alpha}, h\right) \partial \Omega s$ любых $h, h^{\prime} \in \mathscr{H}, \alpha \in R$.

ДоказАтЕЛьство. Для любых $h, h^{\prime} \in \mathscr{H}, \alpha \in R$

$$
\begin{aligned}
0 & =d \psi\left(h, h^{\prime}, e_{\alpha}\right) \\
& =\left[\psi\left(h, h^{\prime}\right), e_{\alpha}\right]+\left[\psi\left(h, e_{\alpha}\right), h^{\prime}\right]+\left[\psi\left(h^{\prime}, e_{\alpha}\right), h\right]+\psi\left(\left[h, e_{\alpha}\right], h^{\prime}\right)+\psi\left(\left[h^{\prime}, e_{\alpha}\right], h\right) \\
& =\left[\psi\left(h, h^{\prime}\right), e_{\alpha}\right]+\alpha\left(h^{\prime}\right) \psi\left(h, e_{\alpha}\right)+\alpha(h) \psi\left(h^{\prime}, e_{\alpha}\right)+\alpha(h) \psi\left(h^{\prime}, e_{\alpha}\right)+\alpha\left(h^{\prime}\right) \psi\left(h, e_{\alpha}\right) \\
& =\left[\psi\left(h, h^{\prime}\right), e_{\alpha}\right] .
\end{aligned}
$$

Следовательно, $\psi\left(h, h^{\prime}\right) \in Z(L)$.

Из леммы 5 получаем, что

$$
\begin{aligned}
0 & =d \psi\left(e_{\alpha}, e_{-\alpha}, h\right)=\left[\psi\left(e_{\alpha}, h\right), e_{-\alpha}\right]+\left[\psi\left(e_{-\alpha}, h\right), e_{\alpha}\right]+\psi\left(\left[e_{\alpha}, e_{-\alpha}\right], h\right) \\
& =\left(\widehat{\psi}\left(e_{\alpha}, h\right)+\widehat{\psi}\left(e_{-\alpha}, h\right)\right) h_{\alpha}+\psi\left(h_{\alpha}, h\right) .
\end{aligned}
$$

Так как $\psi\left(h_{\alpha}, h\right) \in Z(L)$, то из последнего равенства и следствия 2 леммы 3 получаем, что $\psi\left(h_{\alpha}, h\right)=0$ и $\widehat{\psi}\left(e_{\alpha}, h\right)=\widehat{\psi}\left(e_{-\alpha}, h\right)$.

В $L$ определено корневое разложение $L=\mathscr{H} \oplus \bigoplus_{\gamma \in R} L_{\gamma}$, где $L_{\gamma}=\left\langle e_{\gamma}\right\rangle_{K}$. С помощью корневого разложения в $L$ можно получить градуировку вида

$$
L=L_{-2}+L_{-1}+L_{0}+L_{1}+L_{2}
$$


где $L_{0}=M_{0}+I, M_{0}$ - алгебра Ли классического типа ранга $l-1, \operatorname{dim} I \leqslant 1$.

Действительно, пусть $\alpha \in B$ и $\alpha$ связан в схеме Дынкина только с одним из корней $B$. Положим $L_{0}=\mathscr{H} \oplus \bigoplus_{\gamma \in R_{0}} L_{\gamma}, L_{i}=\bigoplus_{\gamma \in R_{i}} L_{\gamma}, i \in\{-2,-1,1,2\}$, $R_{i}$ - подмножество корней из $R$, в разложение которых по базису корень $\alpha$ входит с коэффициентом $i$. Корень $\alpha$ можно выбрать таким образом, что $\operatorname{dim} L_{ \pm 2} \leqslant 1$.

Перенумеруем $B$ так, что $\alpha=\alpha_{1}$ и $\alpha_{1}$ связан в схеме Дынкина только с $\alpha_{2}$. Тогда $\left\{\alpha_{2}, \ldots, \alpha_{l}\right\}=B_{0}$ - базис $R_{0}$.

Если $L$ - алгебра типа $\bar{A}_{l}, l+1 \equiv 0(2), \bar{D}_{l}$ или $\bar{E}_{7}$, то $L_{0}=M_{0}$. Во всех других случаях $L_{0}=M_{0}+\left\langle h_{0}\right\rangle, h_{0} \in \mathscr{H}$.

Легко проверяется, что $L_{ \pm 1}-$ неприводимые $L_{0}$-модули. Это является прямьм следствием того, что структурные константы алгебр Ли с однородной системой корней равны $0, \pm 1$.

Всюду далее корни из $B$ будут занумерованы согласно таблице 1.

ТАБЛИЦА 1

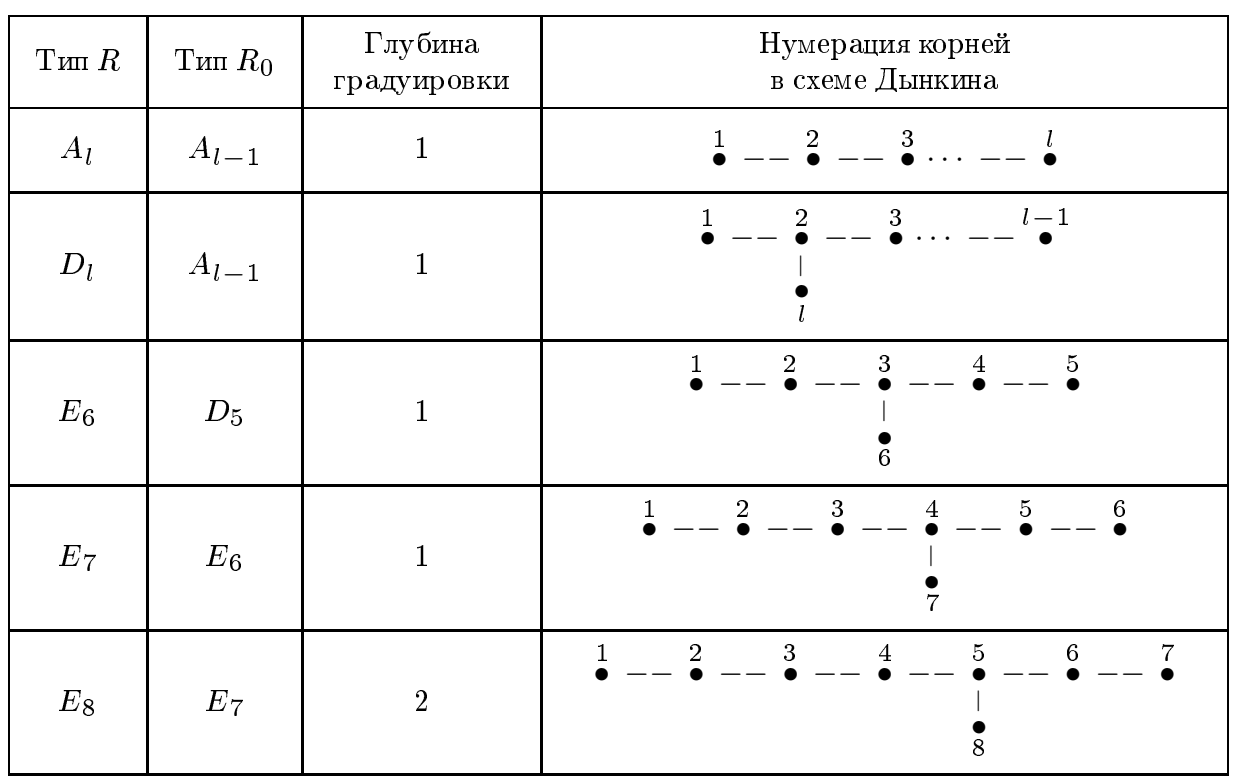

Опишем центр $Z(L)$ алгебры Ли $L$.

ПРЕДЛОЖЕНИЕ 1. (1) Eсли $L$ uмеeт mun $A_{l}, l \equiv 0(2), E_{6}$ uлu $E_{8}$, mo $Z(L)=0$.

(2) Eсли $L$ uмеeт mun $A_{l}, l \equiv 1(2)$, mo $Z(L)=\left\langle h_{1}+h_{3}+\cdots+h_{l-2}+h_{l}\right\rangle_{K}$.

(3) Ecли $L$ uмeеm mun $D_{l}, l \equiv 1(2)$, mo $Z(L)=\left\langle h_{1}+h_{l}\right\rangle_{K}$.

(4) Ecли $L$ имеет mun $D_{l}, l \equiv 0(2)$, mo $Z(L)=\left\langle h_{1}+h_{l}, h_{1}+h_{3}+\cdots+\right.$ $\left.h_{l-3}+h_{l-1}\right\rangle_{K}$.

(5) Ecли $L$ имеeт mun $E_{7}$, mo $Z(L)=\left\langle h_{1}+h_{3}+h_{7}\right\rangle_{K}$.

ДоКАЗАТЕЛЬСТво. Из следствия 1 леммы 3 получаем, что $Z(L) \subset \mathscr{H}$ и, следовательно, является ядром матрицы Картана. 
Доказательство многих утверждений будет проводиться индукцией по рангу алгебры $L$. Поэтому сначала докажем

ПреДЛОЖЕНИЕ 2. Если L имеeт mun $A_{2}$, mo $H^{2}(L, L)=0$.

ДокАЗАТЕльСтво. Пусть $U(L)$ - универсальная обертывающая алгебра. Введем обозначения: $e_{1}=e_{\alpha_{1}}, e_{2}=e_{\alpha_{2}}, e_{3}=e_{\alpha_{1}+\alpha_{2}}, f_{1}=e_{-\alpha_{1}}, f_{2}=e_{-\alpha_{2}}$, $f_{3}=e_{-\alpha_{1}-\alpha_{2}}$.

Рассмотрим элемент $u=e_{1} f_{1}+e_{2} f_{2}+e_{3} f_{3}+h_{1} h_{2} \in U(L)$. Покажем, что $u \in Z(U(L))$. Так как множество $\left\{e_{1}, e_{2}, f_{1}, f_{2}\right\}$ порождает $L$ и $u=f_{1} e_{1}+f_{2} e_{2}+$ $f_{3} e_{3}+h_{1} h_{2}$, то достаточно установить, что $\left[u, e_{1}\right]=\left[u, e_{2}\right]=0$. Это проверяется непосредственно. При присоединенном представлении $u$ действует на $L$ как тождественный оператор.

Как известно (см., например, [11; гл. $2, \S 5$, предложение 4.2$])$, из существования элемента с такими свойствами следует, что $H^{*}(L, L)=0$.

\section{§3. Вычисление $H_{0}^{2}(L, L)$}

В этом параграфе индукцией по рангу доказывается тривиальность группы $H_{0}^{2}(L, L)$. Индукция начинается с алгебры Ли типа $A_{2}$. Если $L$ имеет тип $A_{2}$, то $H_{0}^{2}(L, L)=0$ согласно предложению 2 .

Пусть $L$ - алгебра Ли ранга $l>2$. Предположим, что $H_{0}^{2}(L, L)=0$ для алгебр $L$ ранга меньше $l$. Выберем коцикл $\psi \in Z_{0}^{2}(L, L)$. Обозначим $L \otimes K[\varepsilon]$, где $K[\varepsilon]$ - алгебра двойных чисел, через $L(\varepsilon)$ и отождествим пространство $L$ с $L \otimes 1$. На $L(\varepsilon)$ можно определить структуру алгебры Ли $(L(\varepsilon),[\cdot, \cdot])$, если продолжить лиевское умножение с $L$. При помощи коцикла $\psi$ определим на $L(\varepsilon)$ новую структуру алгебры Ли, полагая $x \circ y=[x, y]+\varepsilon \psi(x, y), x, y \in L$. Очевидно, коцикл $\psi$ когомологичен нулю тогда и только тогда, когда алгебры Ли $(L(\varepsilon),[\cdot, \cdot])$ и $(L(\varepsilon)$, о $)$ изоморфны. В дальнейшем через $L(\varepsilon)$ будем обозначать алгебру Ли $(L(\varepsilon), \circ)$.

Так как коцикл $\psi$ имеет нулевой вес, то в $L(\varepsilon)$ можно построить градуировку по системе корней, как это сделано для $L$ в $\S 2$. Для $\gamma \in R$ положим $L_{\gamma}(\varepsilon)=L_{\gamma} \otimes K[\varepsilon]$;

$$
L(\varepsilon)=L_{-2}(\varepsilon)+L_{-1}(\varepsilon)+L_{0}(\varepsilon)+L_{1}(\varepsilon)+L_{2}(\varepsilon),
$$

где

$$
L_{0}(\varepsilon)=(\mathscr{H} \otimes K[\varepsilon]) \oplus \bigoplus_{\gamma \in R_{0}} L_{\gamma}(\varepsilon), \quad L_{i}(\varepsilon)=\bigoplus_{\gamma \in R_{i}} L_{\gamma}(\varepsilon), \quad i \in\{-2,-1,1,2\},
$$

$R_{i}$ - подмножества системы $R$ (см. таблицу 1 ).

Далее покажем, что алгебра Ли $L_{0}(\varepsilon)$ изоморфна $L_{0} \otimes K[\varepsilon]$ с умножением $[\cdot, \cdot \cdot]$.

Рассмотрим коцепи $\bar{\varphi}_{i}=h_{1}^{*} \otimes h_{i}, i=1, \ldots, l$, и определим отображения $\varphi_{i}: L \rightarrow L$, полагая $\varphi_{i}(x)=d \bar{\varphi}_{i}\left(h_{1}, x\right)$ при $x \in L$.

Лемма 7. Если матрица Картана системъ $R$ невырождена, то $\varphi_{1}, \ldots, \varphi_{l}$ линейно независимъ. 
ДокАЗАТЕЛЬСтво. Пусть $\varphi=a_{1} \varphi_{1}+\cdots+a_{l} \varphi_{l}, a_{i} \in K$. Так как $\varphi_{i}\left(e_{\gamma}\right)=$ $d \bar{\varphi}_{i}\left(h_{1}, e_{\gamma}\right)=\left[\bar{\varphi}_{i}\left(h_{1}\right), e_{\gamma}\right]=\left[h_{i}, e_{\gamma}\right]$, то $\varphi\left(e_{\gamma}\right)=0$ тогда и только тогда, когда $\left[a_{1} h_{1}+\cdots+a_{l} h_{l}, e_{\gamma}\right]=0$. По условию матрица Картана невырождена, следовательно $\varphi_{1}, \ldots, \varphi_{l}$ линейно независимы.

Первым шагом доказательства тривиальности $H_{0}^{2}(L, L)$ будет

ЛЕмма 8. Пусть $l>2, L_{0}-$ нулевая компонента в градуировке алгебры Ли $L, L_{0}=M_{0}+\left\langle h_{1}\right\rangle$ (прямая сумма векторных пространств), $\psi \in H_{0}^{2}(L, L)$. Тогда $\psi$ когомологичен кочиклу $\widetilde{\psi}$ со следующими свойствами:

(1) если матрица Картана $R$ невырождена, то $\widetilde{\psi}\left(h_{1}, x\right)=0$ для всех $x \in L$

(2) если матрица Картана $R$ вырождена, то $\widetilde{\psi}\left(h_{1}, x\right)=0$ для всех $x \in$ $M_{0}$

(3) если $H_{0}^{2}\left(M_{0}, M_{0}\right)=0$, то $\widetilde{\psi}(x, y)=0$ при $x, y \in L_{0}$.

ДокАЗАтЕльство. Если $L$ имеет тип $\bar{A}_{l}, \bar{D}_{l}$ или $\bar{E}_{7}$, то $L_{0}=M_{0}$ и утверждения (2) и (3) очевидны.

Пусть $L$ имеет тип $A_{l}, D_{l}, E_{6}, E_{7}$ или $E_{8}, h=h_{1}$.

(1) Для каждого $\chi \in Z_{0}^{2}(L, L)$ определим коцеп $\chi^{h}=\sum_{\gamma \in R} \widehat{\chi}\left(h, e_{\gamma}\right) h^{*} \wedge$ $e_{\gamma}^{*} \otimes e_{\gamma}$. Очевидно, $\chi$ можно представить в виде $\chi=\chi^{h}+\chi_{1}$, где $\chi_{1}\left(h, e_{\gamma}\right)=0$ для любого $\gamma \in R$. Найдем коцепь $\varphi$ такую, что $(d \varphi)^{h}=\psi^{h}$.

Через $U^{h}(R)$ обозначим подпространство в $C_{0}^{2}(L, L)$, которое является линейной оболочкой системы $\left\{\chi^{h}, \chi \in Z_{0}^{2}(L, L)\right\}$.

Применяя лемму 5 при $\gamma, \delta, \gamma+\delta \in R$, для любого $\chi \in Z_{0}^{2}(L, L)$ имеем

$$
\begin{aligned}
0 & =d \chi\left(e_{\gamma}, e_{\delta}, h\right)=\left[\chi\left(h, e_{\gamma}\right), e_{\delta}\right]+\left[\chi\left(h, e_{\delta}\right), e_{\gamma}\right]+\chi\left(h, e_{\gamma+\delta}\right) \\
& =\left(\widehat{\chi}\left(h, e_{\gamma}\right)+\widehat{\chi}\left(h, e_{\delta}\right)+\widehat{\chi}\left(h, e_{\gamma+\delta}\right)\right) e_{\gamma+\delta} .
\end{aligned}
$$

Таким образом, для любых $\gamma, \delta \in R$ таких, что $\gamma+\delta \in R$, выполняется равенство

$$
\widehat{\chi}\left(h, e_{\gamma}\right)+\widehat{\chi}\left(h, e_{\delta}\right)+\widehat{\chi}\left(h, e_{\gamma+\delta}\right)=0 .
$$

С учетом леммы 6 из равенства (1) получаем, что $\widehat{\chi}\left(h, e_{\gamma}\right)$ для любого $\gamma \in R$ линейно выражается через $\widehat{\chi}\left(h, e_{\alpha_{i}}\right), i=1, \ldots, l$ (выражение не зависит от $\chi$ ). Поэтому $\operatorname{dim} U^{h}(R) \leqslant l$. С другой стороны, из леммы 7 следует, что $d \bar{\varphi}_{1}^{h}, \ldots, d \bar{\varphi}_{l}^{h}$ (в обозначениях леммы 7$)$ линейно независимы. Получаем, что $U^{h}(R)$ порождается $d \bar{\varphi}_{1}^{h}, \ldots, d \bar{\varphi}_{l}^{h}$. Следовательно, $\psi^{h}=b_{1} d \bar{\varphi}_{1}^{h}+\cdots+b_{l} d \bar{\varphi}_{l}^{h}=\left(d\left(b_{1} \bar{\varphi}_{1}+\cdots+b_{l} \bar{\varphi}_{l}\right)\right)^{h}$ для некоторых $b_{i} \in K$. Положим $\varphi=b_{1} \bar{\varphi}_{1}+\cdots+b_{l} \bar{\varphi}_{l}, \quad \widetilde{\psi}=\psi+d \varphi$. Тогда $\widetilde{\psi}\left(h, e_{\gamma}\right)=0$ для любого $\gamma \in R$. Так как по лемме $6 \widetilde{\psi}\left(h^{\prime}, h^{\prime \prime}\right)=0$ для любых $h^{\prime}, h^{\prime \prime} \in \mathscr{H}$, то $\widetilde{\psi}(h, x)=0$ для любого $x \in L$

(2) Как в утверждении (1), для $R_{0}$ определим коцепи $\chi^{h}=\sum_{\gamma \in R_{0}} \widehat{\chi}\left(h, e_{\gamma}\right) h^{*} \wedge$ $e_{\gamma}^{*} \otimes e_{\gamma}$ и $U^{h}\left(R_{0}\right)$.

Если $R$ имеет тип $A_{l}, l \equiv 1(2), D_{l}, l \equiv 1(2)$, или $E_{7}$, то $R_{0}$ имеет тип $A_{l-1}$ или $E_{6}$ и матрица Картана для $R_{0}$ невырождена. Тогда из доказательства утверждения (1) следует, что $\left(d \bar{\varphi}_{2}\right)^{h}, \ldots,\left(d \bar{\varphi}_{l}\right)^{h}$ порождают $U^{h}\left(R_{0}\right)$. В качестве $\widetilde{\psi}$ возьмем $\psi+d\left(b_{2} \bar{\varphi}_{1}+\cdots+b_{l} \bar{\varphi}_{l}\right)$ для подходящих $b_{1}, \ldots, b_{l} \in K$. 
Если $R$ имеет тип $D_{l}, l \equiv 0(2)$, то $R_{0}$ имеет тип $A_{l-1}$. В $R_{0}$ содержится подсистема корней типа $A_{l-2}$, матрица Картана которой невырождена. Тогда из доказательства утверждения (1) следует, что система $\left\{(d \varphi)^{h}, \varphi \in C_{0}^{1}(L, L)\right\}$ порождает подпространство в $U^{h}\left(R_{0}\right)$ размерности не менее $l-2$.

Покажем, что $\operatorname{dim} U^{h}\left(R_{0}\right)=l-2$. Так как $\alpha_{1}$ связан в схеме Дынкина только с $\alpha_{2}$, то $\alpha_{1} \pm \alpha_{i} \notin R$ при $i \neq 1,2$. Следовательно,

$0=d \chi\left(e_{\alpha_{1}}, e_{-\alpha_{1}}, e_{\alpha_{i}}\right)=\left[\chi\left(e_{\alpha_{1}}, e_{-\alpha_{1}}\right), e_{\alpha_{i}}\right]+\chi\left(h, e_{\alpha_{i}}\right)=\left(\alpha_{i}(H)+\widehat{\chi}\left(h, e_{\alpha_{i}}\right)\right) e_{\alpha_{i}}$,

где $\chi\left(e_{\alpha_{1}}, e_{-\alpha_{1}}\right)=H \in \mathscr{H}, i \neq 1,2$. Поэтому $\alpha_{i}(H)+\widehat{\chi}\left(h, e_{\alpha_{i}}\right)=0$ при $i \neq 1,2$. Но согласно предложению 1 линейная комбинация $\alpha_{3}+\alpha_{5}+\cdots+\alpha_{l-3}+\alpha_{l-1}+\alpha_{l}$ является нулевой функцией на $\mathscr{H}$. В частности, $\left(\alpha_{3}+\alpha_{5}+\cdots+\alpha_{l-3}+\alpha_{l-1}+\alpha_{l}\right)(H)=0$. Таким образом, $\widehat{\chi}\left(h, e_{\alpha_{3}}\right)+\widehat{\chi}\left(h, e_{\alpha_{5}}\right)+\cdots+\widehat{\chi}\left(h, e_{\alpha_{l}}\right)=0$ и, учитывая, что $\operatorname{dim} U^{h}\left(R_{0}\right) \geqslant l-2$, имеем $\operatorname{dim} U^{h}\left(R_{0}\right)=l-2$ и $U^{h}\left(R_{0}\right)=\left\langle(d \varphi)^{h}, \varphi \in C_{0}^{1}(L, L)\right\rangle_{K}$. Следовательно, найдется $\varphi \in C_{0}^{1}(L, L)$ такой, что $(d \varphi)^{h}=\psi^{h}$. Положим $\widetilde{\psi}=$ $\psi+d \varphi$.

(3) Пусть $\gamma, \delta \in R_{0}$. Если $\gamma+\delta$ является корнем, то $\gamma+\delta \in R_{0}$ и $\psi\left(e_{\gamma}, e_{\delta}\right) \in M_{0}$. Если $\delta=-\gamma$, то $\psi\left(e_{\gamma}, e_{\delta}\right) \in \mathscr{H}$. Покажем, что $\psi$ можно заменить на когомологичный коцикл $\psi_{1}$, для которого $\psi_{1}\left(e_{\gamma}, e_{-\gamma}\right) \in M_{0}$ при $\gamma \in R_{0}$. Так как $\psi \in$ $Z_{0}^{2}(L, L)$, то $\psi\left(e_{\gamma}, e_{-\gamma}\right)=\sum_{i=1}^{l} \widehat{\psi}\left(e_{\gamma}, e_{-\gamma} ; h_{i}\right) h_{i}$, где $\widehat{\psi}\left(e_{\gamma}, e_{-\gamma} ; h_{i}\right) \in \mathbb{F}_{2}$. Если $\gamma$, $\delta, \gamma+\delta \in R_{0}$, то $\left[\psi\left(e_{-\gamma}, e_{-\delta}\right), e_{\gamma+\delta}\right]+\left[\psi\left(e_{-\gamma}, e_{\gamma+\delta}\right), e_{-\delta}\right]+\left[\psi\left(e_{\gamma+\delta}, e_{-\delta}\right), e_{-\gamma}\right]$ cодержится в $M_{0}$. Тогда из условия $d \psi\left(e_{-\gamma}, e_{-\delta}, e_{\gamma+\delta}\right)=0$ следует, что $\psi\left(e_{\gamma}, e_{-\gamma}\right)+$ $\psi\left(e_{\delta}, e_{-\delta}\right)+\psi\left(e_{\gamma+\delta}, e_{-\gamma-\delta}\right)$ также является элементом $M_{0}$. Следовательно,

$$
\widehat{\psi}\left(e_{\gamma}, e_{-\gamma} ; h_{1}\right)+\widehat{\psi}\left(e_{\delta}, e_{-\delta} ; h_{1}\right)+\widehat{\psi}\left(e_{\gamma+\delta}, e_{-\gamma-\delta} ; h_{1}\right)=0
$$

для любых $\gamma, \delta, \gamma+\delta \in R_{0}$. Поэтому $\widehat{\psi}\left(e_{\gamma}, e_{-\gamma} ; h_{1}\right)$ для любого $\gamma \in R_{0}$ линейно выражается через $\widehat{\psi}\left(e_{\alpha_{i}}, e_{-\alpha_{i}} ; h_{1}\right), i=2, \ldots, l$. Рассмотрим коциклы $\chi_{i}=d\left(h_{i}^{*} \otimes h_{1}\right)$, $i=2, \ldots, l$. Так как $\chi_{i}\left(e_{\alpha_{i}}, e_{-\alpha_{i}}\right)=h_{1}$ и $\chi_{j}\left(e_{\alpha_{i}}, e_{-\alpha_{i}}\right)=0$ для любых $i \neq j$, то найдется такая линейная комбинация $\chi=a_{2} \chi_{2}+\cdots+a_{l} \chi_{l}$, что $\widehat{\chi}\left(e_{\gamma}, e_{-\gamma} ; h_{1}\right)=$ $\widehat{\psi}\left(e_{\gamma}, e_{-\gamma} ; h_{1}\right)$ для любого $\gamma \in R_{0}$. Складывая $\psi \mathrm{c} d\left(a_{2} h_{2}^{*} \otimes h_{1}+\cdots+a_{l} h_{l}^{*} \otimes h_{1}\right)$, получаем коцикл $\psi_{1}$ такой, что $\psi_{1}\left(e_{\gamma}, e_{-\gamma}\right) \in M_{0}$ при $\gamma \in R_{0}$. Заменим $\psi$ на $\psi_{1}$.

Рассмотрим ограничение $\psi$ на $M_{0}$. Так как $\psi(x, y) \in M_{0}$ для любых $x, y \in M_{0}$, то $\left.\psi\right|_{M_{0}} \in Z_{0}^{2}\left(M_{0}, M_{0}\right)$. Тогда по условию $H_{0}^{2}\left(M_{0}, M_{0}\right)=0,\left.\psi\right|_{M_{0}}=d^{\prime} \varphi^{\prime}\left(d^{\prime}-\right.$ дифференциал комплекса $\left.C^{\bullet}\left(M_{0}, M_{0}\right)\right)$ для некоторого $\varphi^{\prime} \in C_{0}^{1}\left(M_{0}, M_{0}\right)$. Отображение $\varphi^{\prime}$ можно рассматривать как элемент $C_{0}^{1}(L, L)$. Получаем, что ограничение на $M_{0}$ коцикла $\psi_{2}=\left(\psi+d \varphi^{\prime}\right)$ равно нулю.

Наконец, применим утверждение (1) или (2) к $\psi_{2}$. Получим, что $\widetilde{\psi}_{2}\left(h_{1}, x\right)=0$ для любого $x \in M_{0}$. Так как в утверждениях (1), (2) коцепь $\varphi$ выбиралась как линейная комбинация $h_{1}^{*} \otimes h_{i}$ и $h_{1}$ не содержится в $M_{0}$, то $d \varphi(x, y)=0$ для любых $x, y \in M_{0}$. Следовательно, $\widetilde{\psi}_{2}(x, y)=\psi_{2}(x, y)+d \varphi(x, y)=0$, если $x, y \in M_{0}$. Таким образом, коцикл $\widetilde{\psi}_{2}$ равен нулю на $L_{0}$. Положим $\widetilde{\psi}=\widetilde{\psi}_{2}$.

Согласно лемме 8 по предположению индукции можно считать, что $\left.\psi\right|_{L_{0}}=0$ и алгебра Ли $L_{0} \otimes K[\varepsilon]$ с умножением $[\cdot, \cdot]$ изоморфна $L_{0}(\varepsilon)$ с умножением $\circ$. 
Подалгебру $L_{0} \otimes 1$ отождествим с $L_{0}$. Напомним, что $L_{0}=M_{0}+\left\langle h_{0}\right\rangle, \mathscr{H}=\mathscr{H}_{0}+$ $\left\langle h_{0}\right\rangle$, где $\mathscr{H}_{0}=\left\langle h_{2}, \ldots, h_{l}\right\rangle_{K}-$ подалгебра Картана в $M_{0}$.

Для следуюшего шага доказательства нам понадобятся предложения 4 и 5 из работы [5; $§ 4]$, которые формулируются здесь как предложения 3 и 4 соответственно. Доказательство предложения 4 для алгебр Ли с однородной системой корней может быть упрощено.

ПРЕДЛОЖЕНИЕ 3 . (1) $L_{-1}(\varepsilon)$ является ограниченным $M_{0}$-модулем.

(2) $\psi\left(h, L_{-1}\right)=0$ для любого $h \in \mathscr{H}_{0}$.

ДокАЗАТЕЛЬСТво. Пусть $V=L_{-1}(\varepsilon)$. Как в доказательстве предложения 4 из работы $[5 ; \S 4]$, показываем, что $\widehat{\psi}\left(h_{\alpha}, v\right)$ не зависит от $v \in V$ для любого $\alpha \in R_{0}$.

Так как ранг алгебры больше двух, то для любого $\alpha \in R_{0}$ существует $\gamma \in R_{-1}$ такой, что $\alpha+\gamma \notin R,-\alpha+\gamma \notin R$. Действительно, для $\alpha_{3}$ подходит $-\alpha_{1}$, а для остальных корней из $R_{0}$ существование $\gamma$ следует из транзитивности действия $W\left(R_{0}\right)$ на $R_{0}$ и того, что $W\left(R_{0}\right)\left(-\alpha_{1}\right) \in R_{-1}$.

Пусть $h_{\alpha} \in \mathscr{H}_{0}$, выберем $\gamma \in R_{-1}$, для которого $\alpha+\gamma \notin R,-\alpha+\gamma \notin R$. Тогда, так как $\psi(x, y)=0$ для любых $x, y \in L_{0}, 0=d \psi\left(e_{\alpha}, e_{-\alpha}, e_{\gamma}\right)=\psi\left(h_{\alpha}, e_{\gamma}\right)$. Поскольку $\widehat{\psi}\left(h_{\alpha}, v\right)$ не зависит от $v$, то $\widehat{\psi}\left(h_{\alpha}, v\right)=0$ для любого $v \in L_{-1}$. Тем самьм, доказаны утверждения (1) и (2).

ПРЕДЛОЖЕНИЕ $4[5 ; \S 4$, предложение 5$] . M_{0}$-модуль $L_{-1}(\varepsilon)$ является прямой суммой подмодулей, изоморфных $L_{-1}, L_{-1}(\varepsilon)=L_{-1}^{\prime}+\varepsilon L_{-1}^{\prime}$.

Согласно предложению 4 можно считать, что $\psi\left(L_{-1}, M_{0}\right)=0$. Аналогично, можно считать, что $\psi\left(L_{1}, M_{0}\right)=0$.

Дальнейшее доказательство тривиальности коцикла $\psi$ будет различным для алгебр Ли без центра и для алгебр Ли, центр которых нетривиален.

3.1. Вычисление $H_{0}^{2}(L, L)$ для алгебр Ли без центра. Пусть $L$ - классическая алгебра Ли без центра. Из рассматриваемых алгебр таковьми являются алгебры Ли типа $A_{l}, l \equiv 0(2), \bar{A}_{l}, l \equiv 1(2), \bar{D}_{l}, E_{6}, \bar{E}_{7}$ и $E_{8}$.

Если $L_{-2} \neq 0$, то $\left[L_{-1}, L_{-1}\right]=L_{-2}=\left\langle e_{\gamma}\right\rangle$. Так как $L_{-2}$ одномерно, то $\psi\left(e_{\alpha}, e_{\gamma}\right)=0$ для любого $\alpha \in R_{0}$. Отсюда, учитьвая, что $\psi\left(L_{0}, L_{0}\right)=0$, получаем $0=d \psi\left(e_{\alpha}, e_{-\alpha}, e_{\gamma}\right)=-\psi\left(\left[e_{\alpha}, e_{-\alpha}\right], e_{\gamma}\right)=-\psi\left(h_{\alpha}, e_{\gamma}\right)$. Следовательно, $\psi\left(L_{-2}, M_{0}\right)=0$. Аналогично, $\psi\left(L_{2}, M_{0}\right)=0$.

Для $v_{1}, v_{2} \in L_{-1}$ положим $\left[v_{1}, v_{2}\right]=\left\langle v_{1}, v_{2}\right\rangle_{1} e_{\gamma}$, где $\langle\cdot, \cdot\rangle_{1}-M_{0}$-инвариантная невырожденная кососимметрическая форма на $L_{-1}$. В $L(\varepsilon) v_{1} \circ v_{2} \in L_{-2}(\varepsilon)$ и $v_{1} \circ v_{2}=\left\langle v_{1}, v_{2}\right\rangle_{1} e_{\gamma}+\varepsilon\left\langle v_{1}, v_{2}\right\rangle_{2} e_{\gamma}$, где $\langle\cdot, \cdot\rangle_{2}$ - также $M_{0}$-инвариантная невырожденная кососимметрическая форма на $L_{-1}$. Так как $L_{-1}$ - неприводимьй

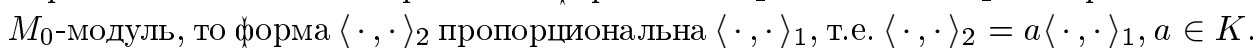
Заменяя $e_{\gamma}$ на $e_{\gamma}+\varepsilon a e_{\gamma}$, получаем $v_{1} \circ v_{2}=\left\langle v_{1}, v_{2}\right\rangle_{1} e_{\gamma}$. Таким образом, можно считать, что $\psi\left(L_{-1}, L_{-1}\right)=0$. Аналогично, подправив базис в $L_{2}(\varepsilon)$, можно считать, что $\psi\left(L_{1}, L_{1}\right)=0$.

Из леммы 8 следует, что к полученным условиям на $\psi$ можно добавить условие $\psi(h, x)=0$ для любых $h \in \mathscr{H}, x \in L$.

Итак, мы доказали, что коцикл $\psi$ когомологичен коциклу, удовлетворяющему условиям $\psi\left(L_{0}, L_{i}\right)=0, i=0, \pm 1, \pm 2, \psi\left(L_{i}, L_{i}\right)=0, i= \pm 1$. 
ПРЕДЛОЖЕНИЕ 5. Пусть $L-$ алгебра Ли без центра. Если $\psi \in Z_{0}^{2}(L, L)$ такой, что $\psi\left(L_{0}, L_{i}\right)=0, i=0, \pm 1, \pm 2, \psi\left(L_{i}, L_{i}\right)=0, i= \pm 1$, то $\psi$ когомологичен нулю.

Доказательство совпадает с доказательством аналогичного предложения, см. $[5 ; \S 4$, предложение 6].

3.2. Вычисление $H_{0}^{2}(L, L)$ для алгебр Ли с ненулевым центром. Согласно предложению 1 если $L$ имеет центр, то алгебра Ли $L$ имеет тип $A_{l}, l+1 \equiv 0(2)$, $D_{l}$ или $E_{7}$. Если алгебра $L$ имеет тип $D_{l}, l \equiv 0(2)$, то размерность центра в $L$ равна 2 , в остальных алгебрах центр одномерньй. Градуировка в $L$ имеет глубину 1 , $L_{ \pm 2}=0$ (см. таблицу 1$)$.

ПрЕДЛОЖЕНИЕ 6 . Пусть $L-$ алгебра Ли одного из типов $A_{l}, l+1 \equiv 0(2)$, $D_{l}$ или $E_{7}, \psi \in Z_{0}^{2}(L, L) . \quad E c л u \psi\left(M_{0}, L_{ \pm 1}\right)=0, \quad \psi\left(L_{i}, L_{i}\right)=0, i=0, \pm 1, m o$ $\psi$ когомологичен нулю.

ДокАЗАТЕЛЬСТво. Покажем, что $\psi$ когомологичен коциклу $\widetilde{\psi}$, для которого при $\alpha \in R_{1}$ выполняется $\widetilde{\psi}\left(e_{\alpha}, e_{-\alpha}\right)=0$.

Пусть $\alpha \in R_{1}, \mathscr{H}_{0}$ - подалгебра с базисом $\left\{h_{2}, \ldots, h_{l}\right\}$. Из леммы 2 следует, что $h_{\alpha}=h_{1}+h$, где $h \in \mathscr{H}_{0}$. Из предложения 1 следует, что $h_{1}=z_{1}+h^{\prime}$, где $h^{\prime} \in \mathscr{H}_{0}, z_{1} \in Z(L) \backslash \mathscr{H}_{0}$. Следовательно, для любого $\alpha \in R_{1} h_{\alpha}=h(\alpha)+z_{1}$, где $h(\alpha) \in \mathscr{H}_{0}, z_{1} \in Z(L) \backslash \mathscr{H}_{0}$.

Положим $\psi\left(e_{\alpha}, e_{-\alpha}\right)=H_{\alpha} \in H$. Далее, выразим $H_{\alpha}$ через $h(\alpha)$ и базис $Z(L)$. Если для некоторого $\gamma \in R_{0} \gamma+\alpha$ и $\gamma-\alpha$ не являются корнями, то из условия $\psi\left(L_{0}, L_{0}\right)=0$ и тождества Якоби для $e_{\alpha}, e_{-\alpha}, e_{\gamma}($ в $L(\varepsilon))$ следует, что $0=\left[h_{\alpha}, e_{\gamma}\right]+\varepsilon\left[H_{\alpha}, e_{\gamma}\right]=\left(\gamma\left(h_{\alpha}\right)+\varepsilon \gamma\left(H_{\alpha}\right)\right) e_{\gamma}$. Таким образом, $h_{\alpha}$ и $H_{\alpha}$ содержатся в ядре функции $\gamma$.

Для любого $\gamma$ из $\left\{\alpha_{3}, \ldots, \alpha_{l}\right\} \subset R_{0} \gamma+\alpha_{1}, \gamma-\alpha_{1}$ не являются корнями. Если $L$ не имеет тип $D_{l}, l \equiv 0(2)$, то из предложения 1 следует, что $\alpha_{3}, \ldots, \alpha_{l}$ линейно независимы как функции на $\mathscr{H}$. Если $L$ имеет тип $D_{l}, l \equiv 0(2)$, то среди $\alpha_{3}, \ldots, \alpha_{l}$ есть $l-3$ линейно независимые функции.

Любой корень $\beta \neq \alpha_{1}$ из $R_{1}$ мы можем получить последовательньм прибавлением к $\alpha_{1}$ корней из базиса $R_{0}$, при этом на каждом шаге мы получаем корни из $R_{1}$. Так как при $\alpha, \beta, \alpha+\beta \in R$ выполняется $\langle\alpha, \beta\rangle=-1$ (лемма 1) и, следовательно, $\alpha+\beta=w_{\beta}(\alpha)$, то $W\left(R_{0}\right) \alpha_{1}=R_{1}$.

Поэтому получаем, что для любого $\alpha \in R_{1}$ сушествуют $l-3$, если $R$ имеет тип $D_{l}, l \equiv 0(2)$, и $l-2$ в остальных случаях линейно независимые функции $\gamma \in R_{0}$ такие, что $\gamma+\alpha, \gamma-\alpha$ не являются корнями.

Таким образом, $h_{\alpha}$ и $H_{\alpha}$ содержатся в пересечении ядер $l-2$ (соответственно $l-3$, если $R$ имеет тип $\left.D_{l}, l \equiv 0(2)\right)$ линейно независимых функций $\gamma$. Так как центр алгебры $L$ также содержится в этом пересечении, то $H_{\alpha}=x_{\alpha} h(\alpha)+y_{\alpha} z_{1}+t_{\alpha} z_{2}$, где $x_{\alpha}, y_{\alpha}, t_{\alpha} \in K,\left\langle z_{1}, z_{2}\right\rangle_{K}=Z(L), z_{1} \notin \mathscr{H}_{0}, z_{2} \in \mathscr{H}_{0}$ (если центр одномерньй, то $\left.z_{2}=0\right)$. Таким образом,

$$
e_{\alpha} \circ e_{-\alpha}=h_{\alpha}+\varepsilon H_{\alpha}=h(\alpha)+z_{1}+\varepsilon\left(x_{\alpha} h(\alpha)+y_{\alpha} z_{1}+t_{\alpha} z_{2}\right) .
$$

Для завершения доказательства предложения применим следуюшее утверждение. 
Лемма 9. Пусть $R$-однородная система корней, $\alpha, \beta \in R_{1}$. Если существует $\gamma \in R_{0}$ такой, что $\alpha=\beta+\gamma$, mо $x_{\alpha}=x_{\beta}, y_{\alpha}=y_{\beta}, t_{\alpha}=t_{\beta}$.

ДокАЗАТЕльство. Из следствия леммы 2 получаем, что $h_{\alpha}=h_{\beta}+h_{\gamma}$. Поэтому $h(\alpha)=h(\beta)+h_{\gamma}$. По условию $\alpha=\beta+\gamma$, следовательно, $\left[e_{\gamma}, e_{-\alpha}\right]=e_{-\beta}$, $\left[e_{\gamma}, e_{\beta}\right]=e_{\alpha}, \quad e_{\beta} \circ e_{-\alpha}=(1+\varepsilon C) e_{-\gamma}$, где $C \in K$. Так как $e_{\gamma} \in L_{0}$, то из тождества Якоби для $e_{\gamma}, e_{\beta}, e_{-\alpha}$ в $L(\varepsilon)$ и условий на коцикл $\psi$ следует, что $(1+\varepsilon C) h_{\gamma}=e_{\alpha} \circ e_{-\alpha}+e_{\beta} \circ e_{-\beta}$. Из равенств $(2)$ и $h(\alpha)=h(\beta)+h_{\gamma}$ получаем, что $(1+\varepsilon C) h_{\gamma}=h_{\gamma}+\varepsilon\left(\left(x_{\beta}+x_{\alpha}\right) h(\beta)+\left(y_{\beta}+y_{\alpha}\right) z_{1}+\left(t_{\alpha}+t_{\beta}\right) z_{2}+x_{\alpha} h_{\gamma}\right)$. Обозначим $x_{\beta}+x_{\alpha}=x_{1}, t_{\alpha}+t_{\beta}=x_{2}$. Тогда так как $z_{1} \notin \mathscr{H}_{0}, h(\beta), z_{2}, h_{\gamma} \in \mathscr{H}_{0}$, то из последнего равенства следует, что $y_{\beta}+y_{\alpha}=0$ и $x_{1} h(\beta)+x_{2} z_{2}+x_{\alpha} h_{\gamma}=C h_{\gamma}$. Так как $h(\beta)=h_{\beta}+z_{1}$, то $x_{1} h_{\beta}+\left(x_{\alpha}+C\right) h_{\gamma}=x_{1} z_{1}+x_{2} z_{2}$. Получаем, что $x_{1} h_{\beta}+\left(x_{\alpha}+C\right) h_{\gamma} \in Z(L)$. Тогда $0=\left[x_{1} h_{\beta}+\left(x_{\alpha}+C\right) h_{\gamma}, e_{\gamma}\right]=x_{1} e_{\gamma}$ (по лемме 1 из условия $\alpha=\beta+\gamma$ следует, что $\langle\beta, \gamma\rangle=-1)$. Следовательно, $x_{1}=0$ и $\left(x_{\alpha}+C\right) h_{\gamma}=x_{2} z_{2} \in Z(L)$. По следствию 2 леммы 3 имеем $x_{2}=0$. Таким образом, $x_{\beta}+x_{\alpha}=0, y_{\alpha}+y_{\beta}=0, t_{\alpha}+t_{\beta}=0$.

Теперь, так как любой корень из $R_{1}$ может быть включен в цепочку, получающуюся последовательньм прибавлением к $\alpha_{1}$ простых корней из $R_{0}$, из леммы 9 следует, что $x_{\alpha}=x_{\beta}=x$ и $y_{\alpha}=y_{\beta}=y, t_{\alpha}=t_{\beta}=t$ для любых $\alpha, \beta \in R_{1}$.

Преобразуем формулу (2):

$$
e_{\alpha} \circ e_{-\alpha}=(1+\varepsilon x)\left(h(\alpha)+\frac{z_{1}+\varepsilon\left(y z_{1}+t z_{2}\right)}{1+\varepsilon x}\right) .
$$

Заменяя базис $\left\{e_{\alpha}, \alpha \in R_{1}\right\}$ на $\left\{(1+\varepsilon x) e_{\alpha}\right\}$ и заменяя $z_{1}$ на $\frac{z_{1}+\varepsilon\left(y z_{1}+t z_{2}\right)}{1+\varepsilon x}$, получаем из равенства (3), что

$$
e_{\alpha} \circ e_{-\alpha}=h(\alpha)+z_{1}=h_{\alpha}
$$

для любого $\alpha \in R_{1}$. Поэтому мы можем считать, что $\psi\left(e_{\alpha}, e_{-\alpha}\right)=0$.

Пусть $\alpha, \beta \in R_{1}$. Если $\alpha-\beta \notin R$, то $\psi\left(e_{\alpha}, e_{-\beta}\right)=0$. Если $\gamma=\alpha-\beta \in R_{0}$, то $\alpha=\beta+\gamma$. Из леммы 1 следует, что $\gamma-\beta \notin R$. Тогда так как $\psi\left(e_{\beta}, e_{-\beta}\right)=0$, $\psi\left(M_{0}, L_{0}\right)=\psi\left(M_{0}, L_{ \pm 1}\right)=0$, то

$$
0=d \psi\left(e_{\beta}, e_{-\beta}, e_{\gamma}\right)=\psi\left(\left[e_{\beta}, e_{\gamma}\right], e_{-\beta}\right)=\psi\left(e_{\alpha}, e_{-\beta}\right)
$$

Следовательно, $\psi\left(e_{\alpha}, e_{-\beta}\right)=0$ для любых $\alpha, \beta \in R_{1}$ и $\psi\left(L_{-1}, L_{1}\right)=0$. Далее, так как $\psi\left(M_{0}, L_{1}\right)=\psi\left(L_{-1}, L_{1}\right)=0$, то

$0=d \psi\left(e_{\alpha}, e_{-\alpha}, e_{\beta}\right)=\psi\left(\left[e_{\alpha}, e_{-\alpha}\right], e_{\beta}\right)=\psi\left(h_{\alpha}, e_{\beta}\right)=\psi\left(h(\alpha)+z_{1}, e_{\beta}\right)=\psi\left(z_{1}, e_{\beta}\right)$.

Следовательно, $\psi\left(\mathscr{H}, L_{1}\right)=0$. Аналогично, $\psi\left(\mathscr{H}, L_{-1}\right)=0$. Объединяя все условия, полученные на коцикл $\psi$, имеем $\psi(L, L)=0$.

Итак, мы установили, что любой коцикл $\psi$ нулевого веса когомологичен нулю и, следовательно, $H_{0}^{2}(L, L)=0$. 


\section{§4. Когомологии ненулевого веса}

Пусть $R$ - однородная система корней ранга $l>2$.

Напомним, что веса $H^{2}(L, L)$ содержатся в $\Lambda_{2}(R)$ - множестве весов $\mu$ пространства $C^{2}(L, L)$ таких, что $\mu(h)=0$ для любого $h \in \mathscr{H}$. Если $\mu \in \Lambda_{2}(R)$, то $\langle\mu, \alpha\rangle \equiv 0(2)$ для любого $\alpha \in R$.

4.1. Описание множества $\Lambda_{2}(R)$. Любой вес $C^{2}(L, L)$ по определению можно представить в виде $\mu=\alpha+\beta+\gamma$, где $\alpha, \beta, \gamma \in R \cup\{0\}$. Так как ранг $R$ больше 2 , то любой элемент множества $\Lambda_{2}(R)$ можно представить в виде суммы трех корней.

ЛЕмма 10. Пусть $\mu \in \Lambda_{2}(R), \mu=\alpha+\beta+\gamma$ для некоторых $\alpha, \beta, \gamma \in R$. Тогда $\mu=2 \delta$ для некоторого $\delta \in R \cup\{0\} \Leftrightarrow S=\langle\alpha, \beta, \gamma\rangle_{\mathbb{R}} \cap R$ - система корней типа $A_{2}$. Если $\delta \neq 0$, то один из корней $\alpha, \beta, \gamma$ совпадает $c \delta$.

ДоказАТЕЛЬСтво. Предположим, что $\mu=2 \delta, \delta \in R \cup\{0\}$. Если $\delta \neq 0$, то $4=\langle 2 \delta, \delta\rangle=\langle\alpha, \delta\rangle+\langle\beta, \delta\rangle+\langle\gamma, \delta\rangle$. Это равенство возможно, только если одно из слагаемых равно 2. Можем считать, что $\langle\alpha, \delta\rangle=2$. Следовательно, $\delta=\alpha$ и $\beta+\gamma=\mu-\alpha=2 \alpha-\alpha=\alpha$. Если $\delta=0$, то $\beta+\gamma=\mu-\alpha=-\alpha$. В любом случае $\langle\alpha, \beta, \gamma\rangle_{\mathbb{R}} \cap R$ имеет тип $A_{2}$.

Пусть $S$ имеет тип $A_{2},\left\{\delta_{1}, \delta_{2}\right\}$ - базис системы $S, \mu=n_{1} \delta_{1}+n_{2} \delta_{2}$ для некотоpых $n_{1}, n_{2} \in \mathbb{Z}$. Так как матрица Картана системы корней типа $A_{2}$ невырождена, то $n_{1}, n_{2} \in 2 \mathbb{Z}$.

Для любого $\sigma \in R$ выполняется $\langle\alpha, \sigma\rangle+\langle\beta, \sigma\rangle+\langle\gamma, \sigma\rangle=\langle\mu, \sigma\rangle \equiv 0(2)$. Если $\alpha= \pm \sigma, \beta= \pm \sigma, \gamma= \pm \sigma$, то $\mu=a \sigma$, где $a \equiv 1(2)$, и, следовательно, $\sigma(h)=0$ для всех $h \in \mathscr{H}$. Это противоречит лемме 3 . Поэтому из леммы 1 получаем, что $|\langle\mu, \sigma\rangle|=|\langle\alpha, \sigma\rangle+\langle\beta, \sigma\rangle+\langle\gamma, \sigma\rangle|<6$ для любого $\sigma \in R$.

Если $\mu$ не пропорционален никакому корню, то, используя [12; гл. VIII, $\S 4$, п. 3 , лемма 10], можно заменить $\mu$ на $w \mu$ для подходящего $w \in W(R)$ так, что $n_{1} n_{2}<0$. Тогда $\left|\left\langle\mu, \delta_{1}\right\rangle\right|=\left|2 n_{1}-n_{2}\right| \geqslant 6$. Получили противоречие. Следовательно, $\mu=k \delta$ для некоторых $k \in 2 \mathbb{Z}, \delta \in R$. Так как $|\langle\mu, \sigma\rangle|<6$ для любого $\sigma \in R$ и, в частности, $|\langle\mu, \delta\rangle|=2 k<6$, то $k=0, \pm 2$.

Определим множества

$$
\begin{aligned}
& M_{1}=\{2 \delta \mid \delta \in R \cup\{0\}\}, \\
& M_{2}=\{\alpha+\beta+\gamma \mid \alpha, \beta, \gamma \in R,\langle\alpha, \beta\rangle=\langle\alpha, \gamma\rangle=\langle\beta, \gamma\rangle=1\} \\
& M_{3}=\{\alpha+\beta \mid \alpha, \beta \in R,\langle\alpha, \beta\rangle=0\} \\
& M_{4}=\{\alpha+\beta+\gamma \mid \alpha, \beta, \gamma \in R,\langle\alpha, \beta\rangle=\langle\alpha, \gamma\rangle=\langle\beta, \gamma\rangle=0\}
\end{aligned}
$$

Любой элемент из $\Lambda_{2}(R)$ принадлежит одному из этих множеств, а именно верно

ПрЕДЛОЖЕНИЕ 7. (1) Если $R$ имеет mun $A_{4}, A_{l}, l>5, E_{6}, E_{8}$, mo $\Lambda_{2}(R)=M_{1}$.

(2) Ecли $R$ имеет mun $A_{3}, D_{l}, l \neq 6$, mo $\Lambda_{2}(R) \subset M_{1} \cup M_{2} \cup M_{3}$.

(3) Ecли $R$ uмеет mun $A_{5}$ или $E_{7}$, mo $\Lambda_{2}(R) \subset M_{1} \cup M_{4}$.

(4) Eсли $R$ имеет mun $D_{6}$, mo $\Lambda_{2}(R) \subset M_{1} \cup M_{2} \cup M_{3} \cup M_{4}$.

(5) Если $\mu \in M_{2} \cap \Lambda_{2}(R)$, то н нельзя представить в виде суммы двух корней и единственным образом можсно представить в виде суммы трех корней. 
(6) Если $\mu \in M_{4} \cap \Lambda_{2}(R)$, то $\mu$ нельзя представить в виде суммы двух корней.

ДоказАтельство. Пусть $\mu \in \Lambda_{2}(R), \mu=\alpha+\beta+\gamma$ для некоторых $\alpha, \beta, \gamma \in R$. Рассмотрим векторное подпространство $A=\langle\alpha, \beta, \gamma\rangle_{\mathbb{R}}$ в $V_{\mathbb{R}}$. Тогда $S=A \cap R$ является системой корней в $A$ ранга не более 3 . Согласно $[9 ;$ гл. VI, $\S 1$, п. 7 , предложение 24] существует базис системы $R$, содержащий базис системы $S$. Пусть $\left\{\delta_{1}, \ldots, \delta_{l}\right\}$ - такой базис. Исследуем строение системы $S$.

Согласно лемме 3 корни являются ненулевьми функциями на $\mathscr{H}$, следовательно, $S$ не может иметь тип $A_{1}$.

Предположим, что $S$ есть прямое произведение систем $A_{2}$ и $A_{1}$. Изменяя нумерацию, можем считать, что $\left\{\delta_{1}\right\}$ - базис системы $A_{1},\left\{\delta_{2}, \delta_{3}\right\}$ - базис системы $A_{2}$. Тогда $\mu=\alpha+\beta+\gamma= \pm \delta_{1}+n_{2} \delta_{2}+n_{3} \delta_{3}$, где $n_{2}, n_{3} \in \mathbb{Z}$. Так как $\mu\left(h_{\delta_{2}}\right)=2 n_{2}-n_{3}$, $\mu\left(h_{\delta_{3}}\right)=-n_{2}+2 n_{3}$, то $n_{2} \equiv n_{3} \equiv 0(2)$. Поскольку $R$ - неприводимая система корней, то сушествует корень $\delta_{i}$, который связан в схеме Дынкина с $\delta_{1}$. Тогда $\mu\left(h_{\delta_{i}}\right)=\delta_{1}\left(h_{\delta_{i}}\right)=1$. Это противоречит тому, что $\left.\mu\right|_{\mathscr{H}}=0$. Следовательно, $S$ не может быть прямым произведением систем $A_{2}$ и $A_{1}$.

Предположим, что $S$ есть прямое произведение систем $A_{1}$ и $A_{1}$. Тогда по крайней мере два из корней $\alpha, \beta, \gamma$ должны быть пропорциональны и $\left.\mu\right|_{\mathscr{H}}$ совпадает с $\left.\alpha\right|_{\mathscr{H}},\left.\beta\right|_{\mathscr{H}}$ или $\left.\gamma\right|_{\mathscr{H}}$. Получаем противоречие лемме 3 . Поэтому $S$ не может иметь тип $A_{1} \times A_{1}$.

Таким образом, $S$ может иметь только тип $A_{3}, A_{2}$ или являться произведением трех копий систем типа $A_{1}$.

Пусть $S$ имеет тип $A_{3},\left\{\delta_{1}, \delta_{2}, \delta_{3}\right\}$ - базис $S$. Тогда $\mu=n_{1} \delta_{1}+n_{2} \delta_{2}+n_{3} \delta_{3}$. Так как $\mu\left(h_{\delta_{1}}\right)=n_{2}$ и $\mu\left(h_{\delta_{2}}\right)=n_{1}+n_{3}$, то $n_{2} \equiv 0(2), n_{1} \equiv n_{3}(2)$. Из вида корней в $A_{3}$ следует, что $\mu=\alpha+\beta+\gamma \in 2 Q(R) \Leftrightarrow \mu=2 \delta$ для некоторого $\delta \in R \cup\{0\}$. Из леммы 10 тогда получаем, что $\mu \notin 2 Q(R)$. Следовательно, $n_{1} \equiv 1(2)$ и $n_{2} \equiv 1(2)$. Если сушествует корень $\delta_{i}$, которьй связан только с $\delta_{1}$ или только с $\delta_{3}$, то $\mu\left(h_{\delta_{i}}\right)=1$. Следовательно, корень $\delta_{i}$ при $i \neq 1,3$ либо связан в схеме Дынкина и с $\delta_{1}$, и с $\delta_{3}$, либо не связан ни с $\delta_{1}$, ни с $\delta_{3}$. Таким образом, $R$ может иметь только тип $A_{3}$ либо $D_{l}$.

Предположим, что $S$ имеет тип $A_{1} \times A_{1} \times A_{1}$. Пусть $\left\{\delta_{1}, \delta_{2}, \delta_{3}\right\}$ - базис системы $S$. Тогда $\mu=\alpha+\beta+\gamma=\varepsilon_{1} \delta_{1}+\varepsilon_{2} \delta_{2}+\varepsilon_{3} \delta_{3}, \varepsilon_{i}= \pm 1$. Если существует корень $\delta_{i}, i \neq 1,2,3$, который связан в схеме Дынкина с $\delta_{1}, \delta_{2}$ и с $\delta_{3}$ или связан только с одним из них, то $\mu\left(h_{\delta_{i}}\right)=1$. Полученное противоречие показьвает, что в схеме Дынкина корни системы $R$ либо не связаны с $\delta_{1}, \delta_{2}, \delta_{3}$, либо связаны ровно с двумя из них. Так как $R$ - неприводимая система, то при таких ограничениях $R$ может иметь только один из типов $A_{5}, D_{6}$ или $E_{7}$.

(1) Если $R$ имеет тип $A_{2}, A_{4}, A_{l}, l>5, E_{6}, E_{8}$, то $S$ имеет тип $A_{2}$ и $\mu \in M_{1}$ по лемме 10.

(2) Если $R$ имеет тип $A_{3}, D_{l}, l \neq 6$, то $S$ имеет тип $A_{2}$ или $A_{3}$. Если $S$ имеет тип $A_{2}$, то $\mu \in M_{1}$ по лемме 10 .

Пусть $S$ имеет тип $A_{3}$. Из леммы 10 следует, что $\mu \neq 2 \delta$ ни для какого $\delta \in R \cup$ $\{0\}$. Тогда либо $\mu=\beta_{1}+\beta_{2}$ для некоторых $\beta_{1}, \beta_{2} \in R, \beta_{1} \neq \pm \beta_{2}$, либо $\mu$ не является суммой двух корней.

Рассмотрим первый случай. Пусть $\mu=\beta_{1}+\beta_{2}, \beta_{1}, \beta_{2} \in R$. Так как корни 
являются ненулевыми функциями на $\mathscr{H}$ (лемма 3 ), то $\beta_{1}+\beta_{2}=\mu$ и $\beta_{1}-\beta_{2}=\mu-2 \beta_{2}$ не являются корнями. Тогда по лемме 1 получаем, что $\left\langle\beta_{1}, \beta_{2}\right\rangle \neq \pm 1$. Так как $\beta_{1} \neq \pm \beta_{2}$, то $\left\langle\beta_{1}, \beta_{2}\right\rangle \neq \pm 2$. Следовательно, $\left\langle\beta_{1}, \beta_{2}\right\rangle=0$ и $\mu \in M_{3}$.

Рассмотрим второй случай. Так как $\mu$ не является суммой двух корней, то $\alpha+\beta$, $\alpha+\gamma, \beta+\gamma$ не являются корнями. Тогда из леммы 1 следует, что $\langle\alpha, \beta\rangle \neq-1$, $\langle\alpha, \gamma\rangle \neq-1,\langle\beta, \gamma\rangle \neq-1$. Так как корни являются ненулевыми функциями на $\mathscr{H}$, то $\langle\alpha, \beta\rangle \neq \pm 2,\langle\alpha, \gamma\rangle \neq \pm 2,\langle\beta, \gamma\rangle \neq \pm 2$.

Если $\langle\alpha, \beta\rangle=0$, то $\langle\mu, \alpha\rangle=2+\langle\gamma, \alpha\rangle$. Следовательно, $\langle\alpha, \gamma\rangle=0$ и $\langle\mu, \gamma\rangle=$ $\langle\beta, \gamma\rangle+2$. Поэтому $\langle\beta, \gamma\rangle=0$. Получили, что $\langle\alpha, \beta\rangle=\langle\alpha, \gamma\rangle=\langle\beta, \gamma\rangle=0$. Это противоречит тому, что $S$ имеет тип $A_{3}$. Следовательно, $\langle\alpha, \beta\rangle=1$. Аналогично, $\langle\alpha, \gamma\rangle=\langle\beta, \gamma\rangle=1$ и $\mu \in M_{2}$.

(3) Если $R$ имеет тип $A_{5}$ или $E_{7}$, то $S$ имеет тип $A_{2}$ или $A_{1} \times A_{1} \times A_{1}$. Если $S$ имеет тип $A_{2}$, то $\mu \in M_{1}$ по лемме 10 . Если $S$ имеет тип $A_{1} \times A_{1} \times A_{1}$, то $\langle\alpha, \beta\rangle=\langle\alpha, \gamma\rangle=\langle\beta, \gamma\rangle=0$ и $\mu \in M_{4}$.

(4) Если $R$ имеет тип $D_{6}$, то $S$ имеет тип $A_{2}, A_{3}$ или $A_{1} \times A_{1} \times A_{1}$. Утверждение (4) тогда следует из доказательства утверждений (2) и (3).

(5) Из условия $\langle\alpha, \beta\rangle=\langle\alpha, \gamma\rangle=\langle\beta, \gamma\rangle=1$ получаем, что $\langle\mu, \alpha\rangle=2+1+1=4$.

Допустим, что $\mu=\alpha^{\prime}+\beta^{\prime}+\gamma^{\prime}$ для некоторых $\alpha^{\prime}, \beta^{\prime}, \gamma^{\prime} \in R$. Из равенства $4=$ $\langle\mu, \alpha\rangle=\left\langle\alpha^{\prime}, \alpha\right\rangle+\left\langle\beta^{\prime}, \alpha\right\rangle+\left\langle\gamma^{\prime}, \alpha\right\rangle$ получаем, что одно из чисел $\left\langle\alpha^{\prime}, \alpha\right\rangle,\left\langle\beta^{\prime}, \alpha\right\rangle,\left\langle\gamma^{\prime}, \alpha\right\rangle$ равно 2. Можно считать, что $\left\langle\alpha^{\prime}, \alpha\right\rangle=2$ и, следовательно, $\alpha=\alpha^{\prime}$. Аналогично, далее показываем, что $\beta=\beta^{\prime}, \gamma=\gamma^{\prime}$.

Если $\mu=\sigma_{1}+\sigma_{2}$ для некоторых $\sigma_{1}, \sigma_{2} \in R$, то $4=\langle\mu, \alpha\rangle=\left\langle\sigma_{1}, \alpha\right\rangle+\left\langle\sigma_{2}, \alpha\right\rangle$. Следовательно, $\left\langle\sigma_{1}, \alpha\right\rangle=\left\langle\sigma_{2}, \alpha\right\rangle=2$ и $\sigma_{1}=\sigma_{2}=\alpha$. Тогда $\mu=2 \alpha$. Аналогично, $\mu=2 \beta$ и $\mu=2 \gamma$. Поэтому $\alpha=\beta=\gamma$. Получили противоречие. Следовательно, $\mu$ нельзя представить в виде суммы двух корней.

(6) Если $\mu=\sigma_{1}+\sigma_{2}$ для некоторых $\sigma_{1}, \sigma_{2} \in R$, то $\langle\mu, \alpha\rangle=\left\langle\sigma_{1}, \alpha\right\rangle+\left\langle\sigma_{2}, \alpha\right\rangle$. $\mathrm{C}$ другой стороны, $\langle\mu, \alpha\rangle=\langle\alpha, \alpha\rangle+\langle\beta, \alpha\rangle+\langle\gamma, \alpha\rangle=2+0+0=2$. Следовательно, $\left\langle\sigma_{1}, \alpha\right\rangle+\left\langle\sigma_{2}, \alpha\right\rangle=2$. Предположим, что $\left\langle\sigma_{1}, \alpha\right\rangle=2$. Тогда $\sigma_{1}=\alpha, \beta+\gamma=\mu-\alpha=$ $\mu-\sigma_{1}=\sigma_{2}$ является корнем, поэтому $\langle\beta, \gamma\rangle=-1$. Получили противоречие, следовательно, $\left\langle\sigma_{1}, \alpha\right\rangle \neq 2$. Точно так же показываем, что $\left\langle\sigma_{2}, \alpha\right\rangle \neq 2$. Тогда поскольку $\left\langle\sigma_{1}, \alpha\right\rangle+\left\langle\sigma_{2}, \alpha\right\rangle=2$, то $\left\langle\sigma_{1}, \alpha\right\rangle=\left\langle\sigma_{2}, \alpha\right\rangle=1$. Аналогично, $\left\langle\sigma_{1}, \beta\right\rangle=$ $\left\langle\sigma_{2}, \beta\right\rangle=1$ и $\left\langle\sigma_{1}, \gamma\right\rangle=\left\langle\sigma_{2}, \gamma\right\rangle=1$. Получаем, что $\left\langle\mu, \sigma_{1}\right\rangle=\left\langle\alpha, \sigma_{1}\right\rangle+\left\langle\beta, \sigma_{1}\right\rangle+$ $\left\langle\gamma, \sigma_{1}\right\rangle=1+1+1=3$. Это противоречит условию $\mu \in \Lambda_{2}(R)$. Поэтому $\mu$ не является суммой двух корней.

Следующие утверждения описывают свойства элементов из $\Lambda_{2}(R)$.

Лемма 11. Пусть $\gamma, \delta, \gamma_{1}, \delta_{1} \in R$ maкuе, что $\langle\gamma, \delta\rangle=0 u \gamma+\delta=\gamma_{1}+\delta_{1}$. Тогда

(1) $\left\langle\gamma_{1}, \delta_{1}\right\rangle=0$, uесли $\{\gamma, \delta\} \neq\left\{\gamma_{1}, \delta_{1}\right\}$, mo $\left\langle\gamma_{1}, \gamma\right\rangle=\left\langle\gamma_{1}, \delta\right\rangle=\left\langle\delta_{1}, \gamma\right\rangle=$ $\left\langle\delta_{1}, \delta\right\rangle=1$

(2) если $R_{ \pm 2}=\varnothing, \gamma+\delta$ является линейной комбинацией корней из $R_{0}, a$ $\gamma, \delta, \gamma_{1}, \delta_{1}$ не содержатся в $R_{0}$, то $\{\gamma, \delta\}=\left\{\gamma_{1}, \delta_{1}\right\}$.

ДоказАтельство. Так как $\gamma_{1}+\delta_{1}=\gamma+\delta$, то $\left\langle\gamma_{1}, \gamma\right\rangle+\left\langle\delta_{1}, \gamma\right\rangle=\langle\gamma, \gamma\rangle+$ $\langle\delta, \gamma\rangle=\langle\gamma, \gamma\rangle=2$. Если $\left\langle\gamma_{1}, \gamma\right\rangle=2$, то $\gamma_{1}=\gamma, \delta_{1}=\delta$. Аналогично, мы получим противоречие условию $\{\gamma, \delta\} \neq\left\{\gamma_{1}, \delta_{1}\right\}$, если предположим, что $\left\langle\gamma_{1}, \gamma\right\rangle=0$. 
Поэтому $\left\langle\gamma_{1}, \gamma\right\rangle=\left\langle\delta_{1}, \gamma\right\rangle=1$. Так как $\gamma_{1}-\gamma=\delta-\delta_{1}$ и $\delta_{1}-\gamma=\delta-\gamma_{1}$, то из леммы 1 теперь следует, что $\left\langle\gamma_{1}, \delta\right\rangle=\left\langle\delta_{1}, \delta\right\rangle=1$. Из условий $\left\langle\gamma, \gamma_{1}\right\rangle=\left\langle\delta, \gamma_{1}\right\rangle=1$ получаем, что $2=\left\langle\gamma+\delta, \gamma_{1}\right\rangle=\left\langle\gamma_{1}+\delta_{1}, \gamma_{1}\right\rangle=2+\left\langle\delta_{1}, \gamma_{1}\right\rangle$. Отсюда $\left\langle\delta_{1}, \gamma_{1}\right\rangle=0$. Утверждение (1) доказано.

Докажем утверждение (2). Если $\{\gamma, \delta\} \neq\left\{\gamma_{1}, \delta_{1}\right\}$, то из утверждения (1) и леммы 1 следует, что $\gamma-\gamma_{1}$ и $\gamma-\delta_{1}$ являются корнями. Так как $R_{ \pm 2}=\varnothing, \gamma, \gamma_{1}, \delta_{1} \in$ $R_{1} \cup R_{-1}$, то $\gamma-\gamma_{1}$ и $\gamma-\delta_{1}$ содержатся в $R_{0}$. Не ограничивая обцности, можем считать, что $\gamma \in R_{1}$. Тогда $\gamma_{1}, \delta_{1}$ также содержатся в $R_{1}$. Это противоречит тому, что $\gamma_{1}+\delta_{1}=\gamma+\delta$ является линейной комбинацией корней из $R_{0}$. Следовательно, $\{\gamma, \delta\}=\left\{\gamma_{1}, \delta_{1}\right\}$.

Лемма 12. Пусть $\mu \in \Lambda_{2}(R), \mu=\delta_{1}+\delta_{2}$, әде $\delta_{1}, \delta_{2} \in R,\left\langle\delta_{1}, \delta_{2}\right\rangle=0 . \quad$ Если $\mu=\gamma_{1}+\gamma_{2}+\gamma_{3}$ для $\gamma_{1}, \gamma_{2}, \gamma_{3} \in R$, mо $\gamma_{1}, \gamma_{2}, \gamma_{3}$ можно перенумеровать так, что $\gamma_{1}+\gamma_{2}, \gamma_{1}+\gamma_{3}$ и $\gamma_{2}-\gamma_{3}$ являются корнями.

ДокАЗАТЕЛЬСТво. Поскольку корни являются ненулевыми функциями на $\mathscr{H}$ (лемма 3) и $\gamma_{1}+\gamma_{2}+\gamma_{3} \in \Lambda_{2}(R)$, то $\gamma_{1} \neq \pm \gamma_{2}, \gamma_{1} \neq \pm \gamma_{3}, \gamma_{2} \neq \pm \gamma_{3}$.

Так как $\mu=\delta_{1}+\delta_{2}, \delta_{1}, \delta_{2} \in R$, то предположения $\left\langle\gamma_{1}, \gamma_{2}\right\rangle=\left\langle\gamma_{1}, \gamma_{3}\right\rangle=\left\langle\gamma_{2}, \gamma_{3}\right\rangle=1$ или $\left\langle\gamma_{1}, \gamma_{2}\right\rangle=\left\langle\gamma_{1}, \gamma_{3}\right\rangle=\left\langle\gamma_{2}, \gamma_{3}\right\rangle=0$ приводят к противоречию с утверждениями (5) или (6) предложения 7. Следовательно, мы можем предположить, что, например, $\left\langle\gamma_{1}, \gamma_{2}\right\rangle=-1$ и $\gamma_{1}+\gamma_{2} \in R$ (лемма 1 ).

Рассмотрим равенство $\mu=\delta_{1}+\delta_{2}=\left(\gamma_{1}+\gamma_{2}\right)+\gamma_{3}$. Так как $\left\langle\delta_{1}, \delta_{2}\right\rangle=0$, то из леммы 11 следует, что $\left\langle\gamma_{1}+\gamma_{2}, \gamma_{3}\right\rangle=0$. Получаем $\left\langle\gamma_{1}, \gamma_{3}\right\rangle=-\left\langle\gamma_{2}, \gamma_{3}\right\rangle$. Если $\left\langle\gamma_{2}, \gamma_{3}\right\rangle=0$, то $\left\langle\mu, \gamma_{2}\right\rangle=\left\langle\gamma_{1}, \gamma_{2}\right\rangle+\left\langle\gamma_{2}, \gamma_{2}\right\rangle+\left\langle\gamma_{3}, \gamma_{2}\right\rangle=-1+2=1$. Это противоречит условию $\mu \in \Lambda_{2}(R)$. Следовательно, $\left\langle\gamma_{2}, \gamma_{3}\right\rangle \neq 0,\left\langle\gamma_{1}, \gamma_{3}\right\rangle \neq 0$. Учитывая условия $\gamma_{1} \neq \pm \gamma_{3}, \gamma_{2} \neq \pm \gamma_{3}$, получаем, что при подходяшей перенумерации $\left\langle\gamma_{1}, \gamma_{3}\right\rangle=-1,\left\langle\gamma_{2}, \gamma_{3}\right\rangle=1$. Тогда из леммы 1 следует, что $\gamma_{1}+\gamma_{3} \in R$ и $\gamma_{2}-\gamma_{3} \in R$.

Лемма 13. Пусть $R$ имеет mun $D_{l}, \mu \in \Lambda_{2}(R), \mu=\gamma_{0}+\delta_{0}$, где $\left\langle\gamma_{0}, \delta_{0}\right\rangle=0$. Тогда

(1) если $l=4$, то $\mu \in M$, где $M=\left\{ \pm\left(\alpha_{1}+\alpha_{3}\right), \pm\left(\alpha_{1}-\alpha_{3}\right), \pm\left(\alpha_{1}+2 \alpha_{2}+\right.\right.$ $\left.\alpha_{3}\right), \pm\left(\alpha_{1}+2 \alpha_{2}+2 \alpha_{4}+\alpha_{3}\right), \pm\left(\alpha_{1}+\alpha_{4}\right), \pm\left(\alpha_{1}-\alpha_{4}\right), \pm\left(\alpha_{1}+2 \alpha_{2}+\alpha_{4}\right), \pm\left(\alpha_{1}+\right.$ $\left.2 \alpha_{2}+2 \alpha_{3}+\alpha_{4}\right), \pm\left(\alpha_{4}+\alpha_{3}\right), \pm\left(\alpha_{4}-\alpha_{3}\right), \pm\left(\alpha_{4}+2 \alpha_{2}+\alpha_{3}\right), \pm\left(\alpha_{4}+2 \alpha_{2}+\right.$ $\left.\left.2 \alpha_{1}+\alpha_{3}\right)\right\}$ - множество всех весов, $W(R)$-сопряженныих с $\alpha_{1}+\alpha_{3}$, $\alpha_{1}+\alpha_{4}, \alpha_{3}+\alpha_{4}$

(2) если $l>4$, то $\mu \in M$, где $M=\left\{ \pm\left(\alpha_{1}-\alpha_{l}\right), \pm\left(\alpha_{1}+\alpha_{l}\right), \pm\left(\alpha_{1}+\alpha_{l}+\right.\right.$ $\left.\left.2 \alpha_{2}\right), \ldots, \pm\left(\alpha_{1}+\alpha_{l}+2 \alpha_{2}+\cdots+2 \alpha_{l-1}\right)\right\}$ - множество всех весов, $W(R)$-сопряженных с $\alpha_{1}+\alpha_{l}$.

ДокАЗАТЕЛЬСТво. Из доказательства утверждения (1) леммы 4 следует сушествование $w \in W(R)$ такого, что $w \gamma_{0}=\alpha_{i}, w \delta_{0}=\alpha_{j}$ для некоторых $i \neq j$. То есть $\mu W(R)$-сопряжен с $\alpha_{i}+\alpha_{j}$ и, следовательно, $\alpha_{i}+\alpha_{j} \in \Lambda_{2}(R)$. Если сушествует простой корень, который связан в схеме Дынкина только с одним из $\alpha_{i}, \alpha_{j}$ или не связан ни с $\alpha_{i}$, ни с $\alpha_{j}$, то получаем противоречие условию $\alpha_{i}+\alpha_{j} \in \Lambda_{2}(R)$. Поэтому в $D_{4}$ имеются три возможности: $\alpha_{i}+\alpha_{j}=\alpha_{1}+\alpha_{3}, \alpha_{i}+\alpha_{j}=\alpha_{1}+\alpha_{4}$, $\alpha_{i}+\alpha_{j}=\alpha_{3}+\alpha_{4}$, а в $D_{l}, l>4,-$ единственная возможность: $\alpha_{i}+\alpha_{j}=\alpha_{1}+\alpha_{l}$. Описание множества $M$ получается прямой проверкой. 
ЛЕмма 14. Пусть $\mu \in \Lambda_{2}(R), \mu=\alpha+\beta+\gamma$, əде $\langle\alpha, \beta\rangle=\langle\alpha, \gamma\rangle=\langle\beta, \gamma\rangle=0$. Тогда

(1) если $R$ имеет тип $A_{5}$, то $\mu \in M$, дде $M-$ множсество всех весов, $W(R)$-сопряженных с $\alpha_{1}+\alpha_{3}+\alpha_{5},|M|=20$

(2) если $R$ имеет тип $D_{6}$, то $\mu \in M$, где $M$ - мнохсество всех весов, $W(R)$-сопряженных с $\alpha_{1}+\alpha_{3}+\alpha_{5}$ или с $\alpha_{6}+\alpha_{3}+\alpha_{5},|M|=64$;

(3) если $R$ имеет mип $E_{7}$, то $\mu \in M$, әде $M$ - множсество всех весов, $W(R)$-сопряжсенных с $\alpha_{1}+\alpha_{3}+\alpha_{7},|M|=56$.

ДокАЗАТЕЛЬСтво. Из доказательства утверждения (2) леммы 4 следует существование $w \in W(R)$ такого, что $\mu=w\left(\alpha_{i}+\alpha_{j}+\alpha_{k}\right)$ для некоторых $i \neq j$, $i \neq k, j \neq k$. Из условия $\mu \in \Lambda_{2}(R)$ следует, что $\alpha_{i}+\alpha_{j}+\alpha_{k} \in \Lambda_{2}(R)$. Поэтому любой корень в схеме Дынкина должен быть связан либо с двумя корнями из $\left\{\alpha_{i}, \alpha_{j}, \alpha_{k}\right\}$, либо не связан ни с одним из них. Таким образом, в $A_{5} \mu$ сопряжен с $\alpha_{1}+\alpha_{3}+\alpha_{5}$, в $D_{6} \mu$ сопряжен либо с $\alpha_{1}+\alpha_{3}+\alpha_{5}$, либо с $\alpha_{6}+\alpha_{3}+\alpha_{5}$, в $E_{7}$ $\mu$ сопряжен с $\alpha_{1}+\alpha_{3}+\alpha_{7}$. Утверждения о мощности множества $M$ получаются прямой проверкой.

\section{2. Вычисление когомологий ненулевого веса.}

ПРЕДЛОЖЕНИЕ 8. Для любого $\sigma \in R \quad H_{2 \sigma}^{2}(L, L)=0$.

ДокАЗАТЕЛЬСтво. Так как все корни $W(R)$-сопряжены с максимальным корнем $\gamma_{0}$, то достаточно рассмотреть $H_{2 \gamma_{0}}^{2}(L, L)$.

Пусть $\psi \in Z_{2 \gamma_{0}}^{2}(L, L)$. Если $h \in \mathscr{H}, \gamma \neq-\gamma_{0}$, то $\psi\left(h, e_{\gamma}\right)=0$. Очевидно, что $\gamma_{0}$ можно представить в виде $\gamma_{0}=\gamma+\delta, \gamma \neq \gamma_{0}, \delta \neq \gamma_{0}$. Тогда из леммы 5 получаем, что

$$
\begin{aligned}
0 & =d \psi\left(e_{-\gamma}, e_{-\delta}, h\right)=\psi\left(\left[e_{-\gamma}, e_{-\delta}\right], h\right)+\left[\psi\left(e_{-\gamma}, h\right), e_{-\delta}\right]+\left[\psi\left(e_{-\delta}, h\right), e_{-\gamma}\right] \\
& =\psi\left(e_{-\gamma_{0}}, h\right)
\end{aligned}
$$

Следовательно, $\psi(\mathscr{H}, L)=0$.

Пусть $2 \gamma_{0}=\alpha+\beta+\gamma$. Из леммы 10 следует, что $S=\langle\alpha, \beta, \gamma\rangle_{\mathbb{R}} \cap R$ является системой корней типа $A_{2}$ и $\gamma_{0} \in\{\alpha, \beta, \gamma\}$. Рассмотрим подалгебру $M$ типа $A_{2}$, порожденную $\left\{e_{\delta}, h_{\delta} \mid \delta \in S\right\}$. Если $\delta_{1}, \delta_{2} \in S, \delta_{1} \neq \delta_{2}$, то либо $\delta_{1}-\delta_{2}+2 \gamma_{0} \notin R \cup\{0\}$, либо $-\delta_{1}-\delta_{2}+2 \gamma_{0} \in S$. Следовательно, $\psi\left(e_{-\delta_{1}}, e_{-\delta_{2}}\right) \in M$ для любых $\delta_{1}, \delta_{2} \in S$. Поэтому $\left.\psi\right|_{M} \in Z_{2 \gamma_{0}}^{2}(M, M)$. Из предложения 2 следует, что $\left.\psi\right|_{M}=d^{\prime} \varphi$, где $d^{\prime}-$ дифференциал комплекса $C^{\bullet}(M, M), \varphi=N e_{\gamma_{0}}^{*} \otimes e_{\gamma_{0}}, N=0,1$. Покажем, что $\psi=d \varphi=N \sum_{-\gamma-\delta+2 \gamma_{0} \in R} e_{-\gamma}^{*} \wedge e_{-\delta}^{*} \otimes e_{-\gamma-\delta+2 \gamma_{0}}, N=0,1$.

Так как $-\alpha-\beta+2 \gamma_{0}=\gamma \in S,-\alpha-\gamma+2 \gamma_{0}=\beta \in S,-\beta-\gamma+2 \gamma_{0}=\alpha \in S$ и $\left.\psi\right|_{M}=d^{\prime} \varphi$, то $\widehat{\psi}\left(e_{-\alpha}, e_{-\beta}\right)=\widehat{\psi}\left(e_{-\alpha}, e_{-\gamma}\right)=\widehat{\psi}\left(e_{-\beta}, e_{-\gamma}\right)$ для любых $\alpha, \beta, \gamma$ таких, что $\alpha+\beta+\gamma=2 \gamma_{0}$.

Допустим, что $2 \gamma_{0}=\alpha+\beta+\gamma=\alpha^{\prime}+\beta^{\prime}+\gamma^{\prime}$, где $\{\alpha, \beta, \gamma\} \neq\left\{\alpha^{\prime}, \beta^{\prime}, \gamma^{\prime}\right\}$. Используя лемму 10, можно считать, что $\gamma_{0}=\gamma=\gamma^{\prime}=\alpha+\beta=\alpha^{\prime}+\beta^{\prime}$. Рассмотрим $d \psi\left(e_{-\alpha}, e_{-\beta}, e_{-\alpha^{\prime}}\right)$. Так как $\gamma_{0}=\alpha+\beta=\alpha^{\prime}+\beta^{\prime}$ и $\{\alpha, \beta\} \neq\left\{\alpha^{\prime}, \beta^{\prime}\right\}$, то из леммы 1 следует, что $\left\langle\gamma_{0}, \alpha\right\rangle=\left\langle\gamma_{0}, \beta\right\rangle=\left\langle\gamma_{0}, \alpha^{\prime}\right\rangle=\left\langle\gamma_{0}, \beta^{\prime}\right\rangle=1$ и $\left\langle\alpha, \alpha^{\prime}\right\rangle \neq 2$, $\left\langle\beta, \alpha^{\prime}\right\rangle \neq 2$. Тогда $1=\left\langle\gamma_{0}, \alpha^{\prime}\right\rangle=\left\langle\alpha+\beta, \alpha^{\prime}\right\rangle=\left\langle\alpha, \alpha^{\prime}\right\rangle+\left\langle\beta, \alpha^{\prime}\right\rangle$ и, следовательно, $\left\langle\alpha, \alpha^{\prime}\right\rangle \neq-1,\left\langle\beta, \alpha^{\prime}\right\rangle \neq-1$. Поэтому $\alpha+\alpha^{\prime} \notin R, \beta+\alpha^{\prime} \notin R$ (лемма 1) и 
$\left[e_{-\alpha}, e_{-\alpha^{\prime}}\right]=\left[e_{-\alpha^{\prime}}, e_{-\beta}\right]=0$. Аналогично, $\alpha+\beta^{\prime} \notin R, \beta+\beta^{\prime} \notin R$. Так как $-\alpha-\alpha^{\prime}+2 \gamma_{0}=\beta+\beta^{\prime},-\beta-\alpha^{\prime}+2 \gamma_{0}=\alpha+\beta^{\prime}$, то $\psi\left(e_{-\alpha}, e_{-\alpha^{\prime}}\right)=\psi\left(e_{-\beta}, e_{-\alpha^{\prime}}\right)=0$. Таким образом, $d \psi\left(e_{-\alpha}, e_{-\beta}, e_{-\alpha^{\prime}}\right)=\left[\psi\left(e_{-\alpha}, e_{-\beta}\right), e_{-\alpha^{\prime}}\right]+\psi\left(\left[e_{-\alpha}, e_{-\beta}\right], e_{-\alpha^{\prime}}\right)=$ $\widehat{\psi}\left(e_{-\alpha}, e_{-\beta}\right)\left[e_{\gamma_{0}}, e_{-\alpha^{\prime}}\right]+\psi\left(e_{-\gamma_{0}}, e_{-\alpha^{\prime}}\right)=\left(\widehat{\psi}\left(e_{-\alpha}, e_{-\beta}\right)+\widehat{\psi}\left(e_{-\gamma_{0}}, e_{-\alpha^{\prime}}\right)\right) e_{\beta^{\prime}}$. Пoэтому $\widehat{\psi}\left(e_{-\alpha}, e_{-\beta}\right)=\widehat{\psi}\left(e_{-\gamma_{0}}, e_{-\alpha^{\prime}}\right)=\widehat{\psi}\left(e_{-\alpha^{\prime}}, e_{-\beta^{\prime}}\right)$ для любых $\alpha, \beta, \alpha^{\prime}, \beta^{\prime} \in R$ таких, что $-\alpha-\beta+2 \gamma_{0} \in R,-\alpha^{\prime}-\beta^{\prime}+2 \gamma_{0} \in R$. Учитывая, что $\psi(\mathscr{H}, L)=0$, получаем $\psi=d \varphi$ и, следовательно, $H_{2 \gamma_{0}}^{2}(L, L)=0$.

Пусть $L$ имеет тип $A_{3}, D_{l}, \bar{A}_{3}$ или $\bar{D}_{l}$. Исследуем пространства $H_{\mu}^{2}(L, L)$, где $\mu=\alpha+\beta+\gamma,\langle\alpha, \beta\rangle=\langle\alpha, \gamma\rangle=\langle\beta, \gamma\rangle=1$.

ПРЕДЛОЖЕНИЕ 9. Пусть $\mu=\alpha+\beta+\gamma \in \Lambda_{2}(R),\langle\alpha, \beta\rangle=\langle\alpha, \gamma\rangle=\langle\beta, \gamma\rangle=1$. Тогда

(1) если $L$ имеет тип $A_{3}, D_{l}$ или $\bar{D}_{l}$, то $H_{\mu}^{2}(L, L)=0$;

(2) если $L$ имеет тип $\bar{A}_{3}$, то $\operatorname{dim} H_{\mu}^{2}(L, L)=1$.

ДоКАЗАТЕЛЬСтво. Пусть $\psi \in H_{\mu}^{2}(L, L)$. Согласно утверждению (5) предложения $7 \mu$ не является суммой двух корней и $\mu=\alpha+\beta+\gamma$-единственное представление $\mu$ в виде суммы трех корней. Поэтому если $\psi(x, y) \neq 0$, то можно считать, что $x=e_{\gamma_{1}}, y=e_{\gamma_{2}}$, где $\left\{\gamma_{1}, \gamma_{2}\right\} \subset\{-\alpha,-\beta,-\gamma\}$. Следовательно,

$$
\begin{aligned}
0 & =d \psi\left(e_{-\alpha}, e_{-\beta}, e_{-\gamma}\right) \\
& =\left[\psi\left(e_{-\alpha}, e_{-\beta}\right), e_{-\gamma}\right]+\left[\psi\left(e_{-\alpha}, e_{-\gamma}\right), e_{-\beta}\right]+\left[\psi\left(e_{-\gamma}, e_{-\beta}\right), e_{-\alpha}\right] \\
& =\widehat{\psi}\left(e_{-\alpha}, e_{-\beta}\right)\left[e_{\gamma}, e_{-\gamma}\right]+\widehat{\psi}\left(e_{-\alpha}, e_{-\gamma}\right)\left[e_{\beta}, e_{-\beta}\right]+\widehat{\psi}\left(e_{-\gamma}, e_{-\beta}\right)\left[e_{\alpha}, e_{-\alpha}\right] \\
& =\widehat{\psi}\left(e_{-\alpha}, e_{-\beta}\right) h_{\gamma}+\widehat{\psi}\left(e_{-\alpha}, e_{-\gamma}\right) h_{\beta}+\widehat{\psi}\left(e_{-\gamma}, e_{-\beta}\right) h_{\alpha} .
\end{aligned}
$$

(1) Если $L$ имеет тип $A_{3}$ или $D_{l}$, то из леммы 4 , следствия 2 леммы 3 и равенства (4) получаем, что $\psi\left(e_{-\alpha}, e_{-\beta}\right)=\psi\left(e_{-\alpha}, e_{-\gamma}\right)=\psi\left(e_{-\gamma}, e_{-\beta}\right)=0$. Следовательно, $\psi=0$.

Пусть $L$ имеет тип $\bar{D}_{l}$. Предположим, что $\alpha=\delta_{1}+\delta_{2}$, где $\delta_{1}, \delta_{2} \in R \backslash\{\beta, \gamma\}$. Рассмотрим $d \psi\left(e_{-\delta_{1}}, e_{-\delta_{2}}, e_{-\beta}\right)$.

По лемме $1-\delta_{1}+\left(-\delta_{2}\right)+\mu=\beta+\gamma \notin R(\langle\beta, \gamma\rangle=1)$ и $\left[\psi\left(e_{-\delta_{1}}, e_{-\delta_{2}}\right), e_{-\beta}\right]=0$. Из равенства $1=\langle\alpha, \beta\rangle=\left\langle\delta_{1}, \beta\right\rangle+\left\langle\delta_{2}, \beta\right\rangle$ и условий $\delta_{1} \neq \beta, \delta_{2} \neq \beta$ получаем, что $\left\langle\delta_{1}, \beta\right\rangle \neq-1$ и $\left\langle\delta_{2}, \beta\right\rangle \neq-1$. Следовательно, $\delta_{1}+\beta \notin R, \delta_{2}+\beta \notin R$ и $\psi\left(\left[e_{-\delta_{1}}, e_{-\beta}\right], e_{-\delta_{2}}\right)=\psi\left(\left[e_{-\beta}, e_{-\delta_{2}}\right], e_{-\delta_{1}}\right)=0$. Аналогично, $\delta_{1}+\gamma \notin R, \delta_{2}+\gamma \notin R$ и, следовательно, $-\delta_{1}+(-\beta)+\mu=\delta_{2}+\gamma$ и $-\delta_{2}+(-\beta)+\mu=\delta_{1}+\gamma$ не являются корнями. Поэтому $\left[\psi\left(e_{-\delta_{1}}, e_{-\beta}\right), e_{-\delta_{2}}\right]=\left[\psi\left(e_{-\delta_{2}}, e_{-\beta}\right), e_{-\delta_{1}}\right]=0$.

Таким образом, если $\alpha=\delta_{1}+\delta_{2}$, причем $\delta_{1} \neq \beta, \gamma, \delta_{2} \neq \beta, \gamma$, то $d \psi\left(e_{-\delta_{1}}\right.$, $\left.e_{-\delta_{2}}, e_{-\beta}\right)=\psi\left(\left[e_{-\delta_{1}}, e_{-\delta_{2}}\right], e_{-\beta}\right)=\psi\left(e_{-\alpha}, e_{-\beta}\right)$. Так как $\psi \in Z_{\mu}^{2}(L, L)$, то получаем, что $\psi\left(e_{-\alpha}, e_{-\beta}\right)=0$. Аналогично, $\widehat{\psi}\left(e_{-\alpha}, e_{-\gamma}\right)=0$. Тогда из равенства (4) и следствия 2 леммы 3 следует, что $\widehat{\psi}\left(e_{-\gamma}, e_{-\beta}\right)=0$. Таким образом, $\psi=0$.

Если система $R$ имеет тип $D_{l}$, то любой корень этой системы можно представить в виде суммы двух корней не менее чем тремя способами. Например, $\alpha_{1}=\left(\alpha_{1}+\right.$ $\left.\cdots+\alpha_{l}\right)+\left(-\alpha_{2}-\cdots-\alpha_{l}\right)=\left(\alpha_{1}+\cdots+\alpha_{l-1}\right)+\left(-\alpha_{2}-\cdots-\alpha_{l-1}\right)=\left(\alpha_{1}+\cdots+\right.$ $\left.\alpha_{l-2}\right)+\left(-\alpha_{2}-\cdots-\alpha_{l-2}\right)$ (см. таблицу 1$)$. Поэтому обязательно сушествуют 
такие корни $\delta_{1}, \delta_{2} \in R$, что $\alpha=\delta_{1}+\delta_{2}$, причем $\delta_{1} \neq \beta, \gamma, \delta_{2} \neq \beta, \gamma$. По ранее доказанному получаем, что $\psi=0$.

(2) Пусть $L$ имеет тип $\bar{A}_{3},\left\{\alpha_{1}, \alpha_{2}, \alpha_{3}\right\}$ - базис системы корней типа $A_{3}$. Прямая проверка показьвает, что $\mu \in\left\{ \pm\left(3 \alpha_{1}+2 \alpha_{2}+\alpha_{3}\right), \pm\left(\alpha_{1}+2 \alpha_{2}+3 \alpha_{3}\right), \pm\left(-\alpha_{1}+2 \alpha_{2}+\right.\right.$ $\left.\left.\alpha_{3}\right), \pm\left(\alpha_{1}+2 \alpha_{2}-\alpha_{3}\right)\right\}$. Так как перечисленные элементы сопряжены относительно групшы Вейля, то достаточно рассмотреть $H_{3 \alpha_{1}+2 \alpha_{2}+\alpha_{3}}^{2}(L, L)$.

Пусть $\alpha=\left(\alpha_{1}+\alpha_{2}+\alpha_{3}\right), \beta=\left(\alpha_{1}+\alpha_{2}\right), \gamma=\alpha_{1}$. Из замечаний, сделанных в начале доказательства, следует, что $\mu=3 \alpha_{1}+2 \alpha_{2}+\alpha_{3}=\alpha+\beta+\gamma-$ единственное представление $\mu$ в виде суммы трех корней и $B_{\mu}^{2}(L, L)=0$. Из равенства (4) получаем, что $\widehat{\psi}\left(e_{-\alpha}, e_{-\beta}\right)=\widehat{\psi}\left(e_{-\alpha}, e_{-\gamma}\right)=\widehat{\psi}\left(e_{-\gamma}, e_{-\beta}\right)$ и, следовательно, $\operatorname{dim} Z_{\mu}^{2}(L, L) \leqslant 1$.

Нетрудно проверить, что $e_{-\alpha}^{*} \wedge e_{-\beta}^{*} \otimes e_{\gamma}+e_{-\alpha}^{*} \wedge e_{-\gamma}^{*} \otimes e_{\beta}+e_{-\gamma}^{*} \wedge e_{-\beta}^{*} \otimes e_{\alpha}$ является элементом $Z_{\mu}^{2}(L, L)$.

Таким образом, $\operatorname{dim} H_{\mu}^{2}(L, L)=\operatorname{dim} Z_{\mu}^{2}(L, L)=1$.

Из утверждений (2), (3) предложения 7 следует, что в когомологиях алгебр Ли типов $A_{3}, \bar{A}_{3}, D_{l}, \bar{D}_{l}$ могут возникать веса вида $\mu=\gamma+\delta,\langle\gamma, \delta\rangle=0$.

Во-первых, исследуем коциклы $\psi$ веса $\mu$ такие, что $\psi(x, h)=0$ для всех $x \in L$, $h \in \mathscr{H}$.

Лемма 15. Пусть $L$ имеет тип $D_{l}$ или $\bar{D}_{l}, \psi \in H_{\mu}^{2}(L, L)$, әде $\mu=\gamma_{0}+\delta_{0}$, $\gamma_{0}, \delta_{0} \in R,\left\langle\gamma_{0}, \delta_{0}\right\rangle=0$. Ecли $l \equiv 1(2)$ u $\psi(x, h)=0$ для всех $x \in L, h \in \mathscr{H}$ или $l \equiv 0(2)$, то для любих $\gamma, \delta \in R$ таких, что $-\gamma-\delta+\mu=0$, выполняется $\psi\left(e_{-\gamma}, e_{-\delta}\right) \in\left\langle h_{\gamma}\right\rangle_{K}+Z(L)$.

ДокаЗАТЕЛЬСтво. Пусть $\mu=\gamma+\delta, \gamma, \delta \in R$. Согласно лемме $11\langle\gamma, \delta\rangle=0$. Так как $-\gamma-\delta+\mu=0$, то $\psi\left(e_{-\gamma}, e_{-\delta}\right)=h^{\prime}$, где $h^{\prime} \in \mathscr{H}$.

Допустим, для $\beta \in R$ выполняется $\langle\beta, \gamma\rangle=\langle\beta, \delta\rangle=0$. Тогда $\mu+\beta-\gamma=\gamma+$ $\delta+\beta-\gamma=\delta+\beta$ и $\mu+\beta-\delta=\gamma+\beta$ не являются корнями и, следовательно, $\psi\left(e_{\beta}, e_{-\gamma}\right)=\psi\left(e_{\beta}, e_{-\delta}\right)=0$. Из условий $\langle\gamma, \delta\rangle=\langle\beta, \gamma\rangle=\langle\beta, \delta\rangle=0$ следует, что $\beta-\gamma, \beta-\delta,-\gamma-\delta$ не являются корнями и, значит, $\psi\left(\left[e_{\beta}, e_{-\gamma}\right], e_{-\delta}\right)=0$, $\psi\left(\left[e_{\beta}, e_{-\delta}\right], e_{-\gamma}\right)=0, \psi\left(\left[e_{-\gamma}, e_{-\delta}\right], e_{\beta}\right)=0$. Поэтому

$$
0=d \psi\left(e_{\beta}, e_{-\gamma}, e_{-\delta}\right)=\left[e_{\beta}, \psi\left(e_{-\gamma}, e_{-\delta}\right)\right]=\left[e_{\beta}, h^{\prime}\right]=\beta\left(h^{\prime}\right) e_{\beta} .
$$

То есть $\beta\left(h^{\prime}\right)=0$, если $\langle\beta, \gamma\rangle=\langle\beta, \delta\rangle=0$.

Пусть $\left\{\alpha_{1}, \ldots, \alpha_{l}\right\}$ - базис системы $R$ (корни занумерованы согласно таблице 1 ). Так же как в доказательстве леммы 13 , заменим $\mu$ на $w \mu$ для подходящего $w \in$ $W(R)$ так, что $\gamma=\alpha_{1}, \delta=\alpha_{l}$. Тогда для любого $\beta \in\left\{\alpha_{3}, \ldots, \alpha_{l-1}\right\}$ выполняется $\langle\beta, \gamma\rangle=\langle\beta, \delta\rangle=0$.

Пусть $l \equiv 0(2)$. Обозначим через $V$ пересечение ядер функций $\alpha_{3}, \ldots, \alpha_{l-1}$. Из предложения 1 следует, что $\alpha_{3}, \ldots, \alpha_{l-1}-$ линейно независимые функции на $\mathscr{H}$ и, следовательно, $\operatorname{dim} V=\operatorname{dim} \mathscr{H}-(l-3)$. Очевидно, что $h_{1}=h_{\gamma} \in V$ и $Z(L) \subset V$. Согласно предложению $1 \operatorname{dim} Z(L)=2$, если $L$ имеет тип $D_{l}$. Тогда $\operatorname{dim} V=3$, если $L$ имеет тип $D_{l}$, и $\operatorname{dim} V=1$, если $L$ имеет тип $\bar{D}_{l}$. Таким образом, $V=\left\langle h_{\gamma}\right\rangle_{K}+Z(L)$. Следовательно, $h^{\prime} \in\left\langle h_{\gamma}\right\rangle_{K}+Z(L)$ и при $l \equiv 0(2)$ утверждение доказано. 
Пусть $l \equiv 1(2)$ и $\psi(x, h)=0$ для всех $x \in L, h \in \mathscr{H}$. Из равенства $d \psi\left(e_{\alpha_{l}}\right.$, $\left.e_{-\alpha_{l}}, e_{-\alpha_{1}}\right)=0\left(\mu=\alpha_{1}+\alpha_{l}\right)$ получаем, что $\left[e_{\alpha_{l}}, \psi\left(e_{-\alpha_{l}}, e_{-\alpha_{1}}\right)\right]=0$ и, следовательно, $\alpha_{l}\left(h^{\prime}\right)=0$. Таким образом, для любого $\beta \in\left\{\alpha_{3}, \ldots, \alpha_{l}\right\}$ вьполняется $\beta\left(h^{\prime}\right)=0$. Обозначим через $V$ пересечение ядер функций $\alpha_{3}, \ldots, \alpha_{l}$. Из предложения 1 следует, что $\alpha_{3}, \ldots, \alpha_{l}$ - линейно независимые функции на $\mathscr{H}$ и, следовательно, $\operatorname{dim} V=\operatorname{dim} \mathscr{H}-(l-2)$. Далее, так же как в случае $l \equiv 0(2)$, получаем, что $h^{\prime} \in\left\langle h_{\gamma}\right\rangle_{K}+Z(L)$.

ПРЕДЛОЖЕНИЕ 10. Пусть L имеeт mun $A_{3}, \bar{A}_{3}, D_{l}, \bar{D}_{l}, \psi \in H_{\mu}^{2}(L, L)$, əдe $\mu=\gamma_{0}+\delta_{0} u\left\langle\gamma_{0}, \delta_{0}\right\rangle=0 . \operatorname{Ecли~} \psi(x, h)=0$ для всех $x \in L, h \in \mathscr{H}$, то верно одно из следующих утверждений:

(1) $\psi$ когомологичен тривиальному коциклу;

(2) L uмеет mun $D_{l}, l \equiv 0(2), u \psi(x, y)=n h_{0}$, где $h_{0} \in Z(L), n \in K, \partial \Omega_{я}$ любъх $x, y \in L \quad\left(h_{0}\right.$ зависит от $\psi$ и не зависит от $\left.x, y\right)$.

ДокАЗАТЕЛЬство. В доказательстве будем использовать ограничения коцикла $\psi$ на подалгебры в алгебре Ли $L$. Сначала выясним, как устроено ограничение $\psi$ на подалгебру типа $A_{2}$.

Пусть $\mu=\gamma_{1}+\gamma_{2}+\gamma_{3}$ для некоторых $\gamma_{1}, \gamma_{2}, \gamma_{3} \in R$. Используя лемму 12 , мы можем перенумеровать $\gamma_{1}, \gamma_{2}, \gamma_{3}$ так, что $\gamma_{1}+\gamma_{2}, \gamma_{1}+\gamma_{3}$ и $\gamma_{2}-\gamma_{3}$ являются корнями. Тогда $L_{\mu}\left(-\gamma_{1},-\gamma_{2}\right)=\left\langle h_{\gamma_{1}}, h_{\gamma_{2}}, e_{ \pm \gamma_{1}}, e_{ \pm \gamma_{2}}, e_{ \pm\left(\gamma_{1}+\gamma_{2}\right)}\right\rangle_{K}$ - подалгебра типа $A_{2}$.

Нетрудно показать, что $\psi\left(e_{\alpha}, e_{\beta}\right)$ при $e_{\alpha}, e_{\beta} \in L_{\mu}\left(-\gamma_{1},-\gamma_{2}\right)$ может быть отлично от нуля, только если $\{\alpha, \beta\}=\left\{-\gamma_{1},-\gamma_{2}\right\},\{\alpha, \beta\}=\left\{\gamma_{1},-\gamma_{1}-\gamma_{2}\right\}$ или $\{\alpha, \beta\}=\left\{-\gamma_{2},-\gamma_{1}-\gamma_{2}\right\}$. Например, $\psi\left(e_{\gamma_{2}}, e_{-\gamma_{1}-\gamma_{2}}\right)=0$, поскольку $\gamma_{2}+\left(-\gamma_{1}-\right.$ $\left.\gamma_{2}\right)+\mu=\gamma_{2}+\gamma_{3}\left(\gamma_{1}, \gamma_{2}, \gamma_{3}\right.$ мы перенумеровали так, что $\gamma_{2}+\gamma_{3}$ не является корнем, $\left.\gamma_{2}+\gamma_{3} \neq 0\right)$. Более того, так как $\psi(x, h)=0$ для всех $x \in L, h \in \mathscr{H}$, то из равенств $d \psi\left(e_{\gamma_{1}}, e_{-\gamma_{1}}, e_{-\gamma_{1}-\gamma_{2}}\right)=0$ и $d \psi\left(e_{\gamma_{2}}, e_{-\gamma_{2}}, e_{-\gamma_{1}-\gamma_{2}}\right)=0$ получаем, что $\widehat{\psi}\left(e_{-\gamma_{1}}, e_{-\gamma_{2}}\right)=\widehat{\psi}\left(e_{\gamma_{1}}, e_{-\gamma_{1}-\gamma_{2}}\right)=\widehat{\psi}\left(e_{-\gamma_{2}}, e_{-\gamma_{1}-\gamma_{2}}\right)$.

Таким образом, если $\mu=\gamma_{1}+\gamma_{2}+\gamma_{3}$ и $\gamma_{1}, \gamma_{2}, \gamma_{3}$ перенумерованы так, что $\gamma_{1}+\gamma_{2}, \gamma_{1}+\gamma_{3}$ и $\gamma_{2}-\gamma_{3}$ являются корнями, то $\left.\psi\right|_{L_{\mu}\left(-\gamma_{1},-\gamma_{2}\right)} \in\left\langle z\left(-\gamma_{1},-\gamma_{2}\right)\right\rangle_{K}$, где $z\left(-\gamma_{1},-\gamma_{2}\right)=e_{-\gamma_{1}}^{*} \wedge e_{-\gamma_{2}}^{*} \otimes e_{\gamma_{3}}+e_{\gamma_{1}}^{*} \wedge e_{-\gamma_{1}-\gamma_{2}}^{*} \otimes e_{\gamma_{1}+\gamma_{3}}+e_{-\gamma_{2}}^{*} \wedge e_{-\gamma_{1}-\gamma_{2}}^{*} \otimes e_{\gamma_{3}-\gamma_{2}}$.

Пусть $\psi\left(e_{-\gamma_{1}}, e_{-\gamma_{2}}\right) \neq 0$ для некоторых $\gamma_{1}, \gamma_{2} \in R$. Если $\psi\left(e_{-\gamma_{1}}, e_{-\gamma_{2}}\right) \notin \mathscr{H}$, то $\gamma_{3}=-\gamma_{1}-\gamma_{2}+\mu \in R$. Так как $\mu=\gamma_{1}+\gamma_{2}+\gamma_{3}$, то из леммы 12 следует, что $\left\langle\gamma_{1}, \gamma_{2}\right\rangle= \pm 1$. Поэтому из леммы 1 следует, что подалгебра, порожденная множеством $\left\{e_{ \pm \gamma_{1}}, e_{ \pm \gamma_{2}}\right\}$, является алгеброй Ли типа $A_{2}$. То есть если $\psi\left(e_{-\gamma_{1}}, e_{-\gamma_{2}}\right)$ не содержится в $\mathscr{H}$, то $e_{-\gamma_{1}}$ и $e_{-\gamma_{2}}$ содержатся в некоторой подалгебре типа $A_{2}$.

Дальнейшее доказательство будем проводить индукцией по рангу $l, l \geqslant 3$.

Пусть $l=3$. В этом случае $L$ имеет тип $A_{3}$ или $\bar{A}_{3}$. Пусть $\left\{\alpha_{1}, \alpha_{2}, \alpha_{3}\right\}$ - базис системы корней типа $A_{3}$. Применив к $\gamma_{0}, \delta_{0}$ подходящее $w \in W(R)$, можно считать, что $\gamma_{0}=\alpha_{1}$. Так как $\left\langle\gamma_{0}, \delta_{0}\right\rangle=0$, то $\delta_{0}= \pm \alpha_{3}$. Следовательно, заменяя $\psi$ на $w \psi$ для подходящего $w \in W(R)$, можем предполагать, что $\mu=\alpha_{1}+\alpha_{3}$.

В алгебрах Ли типов $A_{3}$ и $\bar{A}_{3}$ есть четыре подалгебры типа $A_{2}$ вида $L_{\mu}\left(\gamma_{1}, \gamma_{2}\right)$ для некоторых $\gamma_{1}, \gamma_{2} \in R$, а именно $L_{\mu}\left(\alpha_{1}+\alpha_{2},-\alpha_{1}\right), L_{\mu}\left(-\alpha_{2}-\alpha_{3}, \alpha_{2}\right), L_{\mu}\left(-\alpha_{2}-\right.$ $\left.\alpha_{3},-\alpha_{1}\right), L_{\mu}\left(-\alpha_{1}-\alpha_{2},-\alpha_{3}\right)$.

Пусть $z_{1}=z\left(\alpha_{1}+\alpha_{2},-\alpha_{1}\right), z_{2}=z\left(-\alpha_{2}-\alpha_{3}, \alpha_{2}\right), z_{3}=z\left(-\alpha_{2}-\alpha_{3},-\alpha_{1}\right)$, 
$z_{4}=z\left(-\alpha_{1}-\alpha_{2},-\alpha_{3}\right)$. Согласно доказанному выше $\psi=a_{1} z_{1}+a_{2} z_{2}+a_{3} z_{3}+$ $a_{4} z_{4}+\psi_{1}$, где $a_{1}, a_{2}, a_{3}, a_{4} \in K, \psi_{1}(x, y) \in \mathscr{H}$ для всех $x, y \in L$.

Так как $\left(-\alpha_{1}\right)+\left(-\alpha_{3}\right)+\mu=0$, то $\psi\left(e_{-\alpha_{1}}, e_{-\alpha_{3}}\right)=h \in \mathscr{H}$. Из равенств $d \psi\left(e_{-\alpha_{1}}, e_{-\alpha_{2}}, e_{-\alpha_{3}}\right)=0$ и $d \psi\left(e_{-\alpha_{1}}, e_{\alpha_{2}}, e_{-\alpha_{3}}\right)=0$ получаем соотношения $a_{3}+$ $a_{4}-\alpha_{2}(h)=0$ и $a_{1}+a_{2}+\alpha_{2}(h)=0$ соответственно. Следовательно, $a_{1}+a_{2}+$ $a_{3}+a_{4}=0$. Поэтому мы можем найти такую линейную комбинацию коцепей $\varphi_{1}=d\left(e_{-\alpha_{1}}^{*} \otimes e_{\alpha_{3}}\right)=z_{1}+z_{3}+e_{-\alpha_{1}}^{*} \wedge e_{-\alpha_{3}}^{*} \otimes h_{3}, \varphi_{2}=d\left(e_{-\alpha_{3}}^{*} \otimes e_{\alpha_{1}}\right)=z_{2}+z_{4}+$ $e_{-\alpha_{3}}^{*} \wedge e_{-\alpha_{1}}^{*} \otimes h_{1}, \varphi_{3}=d\left(e_{\alpha_{2}}^{*} \otimes e_{\alpha_{1}+\alpha_{2}+\alpha_{3}}\right)=z_{1}+z_{2}+e_{\alpha_{2}}^{*} \wedge e_{-\alpha_{1}-\alpha_{2}-\alpha_{3}}^{*} \otimes\left(h_{1}+\right.$ $\left.h_{2}+h_{3}\right)$, что $\widetilde{\psi}=\psi+b_{1} \varphi_{1}+b_{2} \varphi_{2}+b_{3} \varphi_{3}$ удовлетворяет условию $\widetilde{\psi}(x, y) \in \mathscr{H}$ для всех $x, y \in L$. Таким образом, коцикл $\psi$ когомологичен коциклу $\widetilde{\psi}$ такому, что $\widetilde{\psi}(x, y) \in \mathscr{H}$ для всех $x, y \in L$. Нетрудно убедиться в том, что коцикл $\widetilde{\psi}$ когомологичен тривиальному коциклу.

Предположим, что предложение верно для алгебр Ли типа $D_{n}$ и $\bar{D}_{n}, n<l$.

Построим в $L$ градуировку, аналогичную определенной в $\S 2$, используя в качестве корня $\alpha$ корень $\alpha_{l-1}$ (см. таблицу 1$), L=L_{-1}+L_{0}+L_{1}, L_{0}=M_{0}+\left\langle h_{0}\right\rangle$, $h_{0} \in \mathscr{H}, M_{0}$ - алгебра Ли типа $D_{l-1}$. Из леммы 13 следует, что, заменяя $\psi$ на $w \psi$ для подходящего $w \in W(R)$ (если $R$ имеет тип $D_{4}$, то используем еше и симметричность диаграммы), можно считать, что $\mu$ является линейной комбинацией корней из $R_{0}$. Тогда если $\gamma, \delta \in R_{0}$, то либо $\gamma+\delta+\mu$ является линейной комбинацией корней из $R_{0}$ и $\psi\left(e_{\gamma}, e_{\delta}\right) \in M_{0}$, либо $\gamma+\delta+\mu=0$. Если $\gamma+\delta+\mu=0$, то из леммы 15 следует, что $\psi\left(e_{\gamma}, e_{\delta}\right) \in\left\langle h_{\gamma}\right\rangle_{K}+Z(L)$. В алгебрах Ли типа $\bar{D}_{l}$ центр равен нулю и $\psi\left(e_{\gamma}, e_{\delta}\right) \in\left\langle h_{\gamma}\right\rangle_{K} \subset M_{0}$. В алгебре Ли типа $D_{l}$ при $l \equiv 1(2)$ центр $L$ содержится в $M_{0}$ (см. предложение 1$)$. Поэтому $\psi\left(e_{\gamma}, e_{\delta}\right)$ также содержится в $M_{0}$. Следовательно, в алгебрах Ли типов $D_{l}, l \equiv 1(2)$, и $\bar{D}_{l}$ ограничение коцикла $\psi$ на $M_{0}$ является коциклом из $Z_{\mu}^{2}\left(M_{0}, M_{0}\right)$ и к нему применимо предположение индукции. Если $L$ имеет тип $D_{l}, l \equiv 0(2)$, то согласно предложению 1 центр $L$ двумерен и сушествует центральный элемент, не содержащийся в $M_{0}$. Поэтому $L_{0}$ является прямой суммой $M_{0} \oplus\left\langle h_{0}\right\rangle, h_{0} \in Z(L)$, и мы можем применить предположение индукции к проекции $\left.\mathrm{pr}\right|_{M_{0}} \psi$ коцикла $\psi$ на $M_{0}$, которая также принадлежит $Z_{\mu}^{2}\left(M_{0}, M_{0}\right)$ (для любых $x, y \in M_{0} \psi(x, y)=\psi^{\prime}(x, y)+a h_{0}$, где $\psi^{\prime}(x, y) \in M_{0}, a \in K, \psi^{\prime}$ называем проекцией $\psi$ на $\left.M_{0}\right)$.

Заменим коцикл $\psi$ на когомологичный коцикл $\psi+d \varphi$, где $\varphi \in C_{\mu}^{1}\left(M_{0}, M_{0}\right)$, такой, что $\left.\psi\right|_{M_{0}}=d^{\prime} \varphi$ или $\left.\mathrm{pr}\right|_{M_{0}} \psi=d^{\prime} \varphi\left(d^{\prime}-\right.$ дифференциал комплекса $\left.C^{\bullet}\left(M_{0}, M_{0}\right)\right)$. Тогда $\psi(x, y) \in\left\langle h_{0}\right\rangle_{K}$ для любых $x, y \in M_{0}$, где $h_{0} \in Z(L) \backslash M_{0}$ (полагаем $h_{0}=0$, если $L$ имеет тип $D_{l}, l \equiv 1(2)$, или $\left.\bar{D}_{l}\right)$.

Покажем, что $\psi(x, y) \in\left\langle h_{0}\right\rangle_{K}$ для любых $x, y \in L$. Если это не выполняется, то для некоторых $\gamma_{1}, \gamma_{2} \in R$ ограничение $\psi$ на подалгебру типа $A_{2}$ вида $L_{\mu}\left(-\gamma_{1},-\gamma_{2}\right)$ отлично от нуля. По определению подалгебры $L_{\mu}\left(-\gamma_{1},-\gamma_{2}\right)$ имеем $-\gamma_{1}-\gamma_{2}+\mu=$ $\gamma_{3} \in R$ и $\gamma_{1}+\gamma_{2} \in R, \gamma_{1}+\gamma_{3} \in R, \gamma_{2}-\gamma_{3} \in R$. Подалгебра $L_{\mu}\left(-\gamma_{1},-\gamma_{2}\right)$ не может целиком содержаться в $M_{0}$, поскольку иначе $\left.\psi\right|_{L_{\mu}\left(-\gamma_{1},-\gamma_{2}\right)}=0$ по предположению индукции. Следовательно, по крайней мере один из корней $\gamma_{1}, \gamma_{2}$ не содержится в $M_{0}$. Тогда так как $\mu$ является комбинацией корней из $R_{0}$ и $\mu=\gamma_{1}+\gamma_{2}+\gamma_{3}$, то ровно два из корней $\gamma_{1}, \gamma_{2}, \gamma_{3}$ не содержатся в $R_{0}$. Поэтому либо $\gamma_{1}+\gamma_{2} \notin R_{0}$, либо $\gamma_{1}+\gamma_{3} \notin R_{0}$.

Если $\gamma_{1}+\gamma_{2} \notin R_{0}$, то $\mu=\gamma_{3}+\left(\gamma_{1}+\gamma_{2}\right)$ - представление $\mu$ в виде суммы двух 
корней, не содержащихся в $M_{0}$. Если $\gamma_{1}+\gamma_{3} \notin R_{0}$, то $\mu=\gamma_{2}+\left(\gamma_{1}+\gamma_{3}\right)$ - представление $\mu$ в виде суммы двух корней, не содержащихся в $M_{0}$. Из утверждения (3) леммы 11 следует, что представление $\mu$ в виде суммы двух корней, не содержашихся в $R_{0}$, единственно с точностью до перестановки слагаемых. Пусть $\mu=\gamma_{0}+\delta_{0}-$ такое представление. Тогда либо $\gamma_{1}+\gamma_{2}$ совпадает с $\gamma_{0}$ или $\delta_{0}$, либо $\gamma_{2}$ совпадает с $\gamma_{0}$ или $\delta_{0}$. В любом случае в $L\left(-\gamma_{1},-\gamma_{2}\right)$ содержится один из $e_{\gamma_{0}}, e_{\delta_{0}}$. Не ограничивая общности, можно предположить, что $e_{\gamma_{0}} \in L\left(-\gamma_{1},-\gamma_{2}\right)$. Следовательно, $L\left(-\gamma_{1},-\gamma_{2}\right)$ можно представить в виде $\left\langle h_{\alpha}, h_{\beta}, e_{ \pm \alpha}, e_{ \pm \beta}, e_{ \pm \gamma_{0}}\right\rangle_{K}$, где $\gamma_{0}=\alpha+\beta$.

Рассмотрим другую подалгебру типа $A_{2}$, содержащую $e_{\gamma_{0}}:\left\{h_{\alpha^{\prime}}, h_{\beta^{\prime}}, e_{ \pm \alpha^{\prime}}, e_{ \pm \beta^{\prime}}\right.$, $\left.e_{ \pm \gamma_{0}}\right\}$, где $\gamma_{0}=\alpha^{\prime}+\beta^{\prime}$ и $\{\alpha, \beta\} \neq\left\{\alpha^{\prime}, \beta^{\prime}\right\}$. Так как $\gamma_{0} \notin R_{0}, \gamma_{0}=\alpha+\beta=\alpha^{\prime}+\beta^{\prime}$, то можно считать, что $\beta, \beta^{\prime} \in R_{0}$. Рассмотрим $d \psi\left(e_{-\alpha}, e_{-\beta}, e_{-\beta^{\prime}}\right)$. Поскольку $\beta, \beta^{\prime} \in R_{0}$, то по предположению индукции $\left[\psi\left(e_{-\beta}, e_{-\beta^{\prime}}\right), e_{-\alpha}\right]=0$. Так как $\gamma_{0}=\alpha+\beta=\alpha^{\prime}+\beta^{\prime}$, то $\left\langle\gamma_{0}, \alpha\right\rangle=\left\langle\gamma_{0}, \beta\right\rangle=\left\langle\gamma_{0}, \alpha^{\prime}\right\rangle=\left\langle\gamma_{0}, \beta^{\prime}\right\rangle=1$. Тогда если $\alpha+\beta^{\prime}$ является корнем, то $\left\langle\alpha+\beta^{\prime}, \gamma_{0}\right\rangle=1+1=2$ и, следовательно, $\alpha+\beta^{\prime}=\gamma_{0}$. Отсюда $\{\alpha, \beta\}=\left\{\alpha^{\prime}, \beta^{\prime}\right\}$. Получили противоречие. Следовательно, $\alpha+\beta^{\prime} \notin R$ и $\left[e_{-\alpha}, e_{-\beta^{\prime}}\right]=0, \psi\left(\left[e_{-\alpha}, e_{-\beta^{\prime}}\right], e_{-\beta}\right)=0$. Аналогично, $\beta+\beta^{\prime} \notin R$ и $\psi\left(\left[e_{-\beta}, e_{-\beta^{\prime}}\right], e_{-\alpha}\right)=0$. Если $\psi\left(e_{-\alpha}, e_{-\beta^{\prime}}\right) \neq 0$, то $\mu=\alpha+\beta^{\prime}+\lambda$, где $\lambda \in R(\lambda \neq 0$, так как $\mu$ является комбинацией корней из $\left.R_{0}\right)$. Так как $\alpha+\beta^{\prime} \notin R$, то из леммы 12 следует, что $\alpha+\lambda \in R, \beta^{\prime}+\lambda \in R$. Тогда $\mu=\alpha+\left(\beta^{\prime}+\lambda\right)-$ представление $\mu$ в виде суммы двух корней, не содержащихся в $R_{0}$. Такое представление единственно, следовательно, $\alpha=\delta_{0}, \lambda+\beta^{\prime}=\gamma_{0}, \mu=\gamma_{0}+\delta_{0}=(\alpha+\beta)+\alpha=2 \alpha+\beta$. Поэтому из условия $\left.\mu\right|_{\mathscr{H}}=0$ получаем, что $\left.\beta\right|_{\mathscr{H}}=0$. Это противоречит лемме 3 . Следовательно, $\psi\left(e_{-\alpha}, e_{-\beta^{\prime}}\right)=0$. Так как $\mu=\gamma_{0}+\delta_{0}=\alpha^{\prime}+\beta^{\prime}+\delta_{0}$, то из леммы 12 следует, что либо $\beta^{\prime}+\delta_{0} \in R$, либо $\beta^{\prime}-\delta_{0} \in R$. Поскольку представление $\mu$ в виде суммы двух корней, не содержащихся в $R_{0}$, единственно и $\beta^{\prime} \neq 0$, то $\beta^{\prime}+\delta_{0} \notin R$. Следовательно, $\beta^{\prime}-\delta_{0} \in R$.

Таким образом,

$$
\begin{aligned}
d \psi\left(e_{-\alpha}, e_{-\beta}, e_{-\beta^{\prime}}\right) & =\left[\psi\left(e_{-\alpha}, e_{-\beta}\right), e_{-\beta^{\prime}}\right]+\psi\left(\left[e_{-\alpha}, e_{-\beta}\right], e_{-\beta^{\prime}}\right) \\
& =\left(\widehat{\psi}\left(e_{-\alpha}, e_{-\beta}\right)+\widehat{\psi}\left(e_{-\alpha-\beta}, e_{-\beta^{\prime}}\right)\right) e_{\delta_{0}-\beta^{\prime}}
\end{aligned}
$$

Следовательно, $\widehat{\psi}\left(e_{-\alpha}, e_{-\beta}\right)=\widehat{\psi}\left(e_{-\gamma_{0}}, e_{-\beta^{\prime}}\right)$.

То есть если ограничение $\psi$ на $L\left(-\gamma_{1},-\gamma_{2}\right)$ отлично от нуля, то ограничение $\psi$ на любую подалгебру $\left\langle h_{\alpha^{\prime}}, h_{\beta^{\prime}}, e_{ \pm \alpha^{\prime}}, e_{ \pm \beta^{\prime}}, e_{ \pm \gamma_{0}}\right\rangle_{K}$, содержащую $e_{\gamma_{0}}$, также отлично от нуля. Аналогичный результат верен и для подалгебр $\left\langle h_{\alpha^{\prime}}, h_{\beta^{\prime}}, e_{ \pm \alpha^{\prime}}, e_{ \pm \beta^{\prime}}\right.$, $\left.e_{ \pm \delta_{0}}\right\rangle_{K}$, содержаших $\delta_{0}$. Поэтому, складывая $\psi$ с линейной комбинацией

$$
d\left(e_{-\gamma_{0}}^{*} \otimes e_{\delta_{0}}\right) \text { и } d\left(e_{-\delta_{0}}^{*} \otimes e_{\gamma_{0}}\right),
$$

получаем коцикл $\widetilde{\psi}$ такой, что $\widetilde{\psi}\left(e_{\gamma_{1}}, e_{\gamma_{2}}\right)=0$ для всех $\gamma_{1}, \gamma_{2} \in R$ таких, что $\gamma_{1}+\gamma_{2}+\mu \neq 0$. Заменим коцикл $\psi$ на когомологичный ему коцикл $\widetilde{\psi}$.

Мы показали, что $\psi\left(e_{\gamma_{1}}, e_{\gamma_{2}}\right)=0$ для всех $\gamma_{1}, \gamma_{2} \in R$ таких, что $\gamma_{1}+\gamma_{2}+\mu \neq 0$. Также по предположению индукции $\psi\left(e_{\gamma_{1}}, e_{\gamma_{2}}\right) \in\left\langle h_{0}\right\rangle_{K}$ для всех $\gamma_{1}, \gamma_{2} \in R_{0}$ таких, что $\gamma_{1}+\gamma_{2}+\mu=0$. Так как $\mu$ является линейной комбинацией корней из $R_{0}$ и $\mu=\gamma_{0}+\delta_{0}$ - единственное представление $\mu$ в виде суммы двух корней, не содержащихся в $R_{0}$, то достаточно показать, что $\psi\left(e_{-\gamma_{0}}, e_{-\delta_{0}}\right) \in\left\langle h_{0}\right\rangle_{K}$. 
Представим $\gamma_{0}$ в виде суммы двух корней $\gamma_{0}=\alpha+\beta$, где $\alpha \notin R_{0}, \beta \in R_{0}$. Рассмотрим $d \psi\left(e_{-\alpha}, e_{-\beta}, e_{-\delta_{0}}\right)$. Если $\beta+\delta_{0} \in R$, то $\mu=\alpha+\left(\beta+\delta_{0}\right)-$ представление $\mu$ в виде суммы двух корней, не содержащихся в $R_{0}$. Так как $\beta \neq 0$, то $\alpha=\delta_{0}, \beta+\delta_{0}=\gamma_{0}, \mu=\alpha+\beta+\delta_{0}=2 \delta_{0}+\beta$. Поэтому $\left.\beta\right|_{\mathscr{H}}=0$, что противоречит лемме 3 . Следовательно, $\beta+\delta_{0} \notin R$ и $\psi\left(\left[e_{-} \delta_{0}, e_{-\beta}\right], e_{-\alpha}\right)=0$. Слагаемые $\left[\psi\left(e_{-\alpha}, e_{-\beta}\right), e_{-\delta_{0}}\right],\left[\psi\left(e_{-\alpha}, e_{-\delta_{0}}\right), e_{-\beta}\right],\left[\psi\left(e_{-\delta_{0}}, e_{-\beta}\right), e_{-\alpha}\right]$ равны нулю, так как $-\alpha-\beta+\mu \neq 0,-\alpha-\delta_{0}+\mu \neq 0$ и $-\delta_{0}-\beta+\mu \neq 0$.

Таким образом,

$$
\begin{aligned}
0 & =d \psi\left(e_{-\alpha}, e_{-\beta}, e_{-\delta_{0}}\right)=\psi\left(\left[e_{-\alpha}, e_{-\beta}\right], e_{-\delta_{0}}\right)+\psi\left(\left[e_{-\alpha}, e_{-\delta_{0}}\right], e_{-\beta}\right) \\
& =\psi\left(e_{-\gamma_{0}}, e_{-\delta_{0}}\right)+\psi\left(\left[e_{-\alpha}, e_{-\delta_{0}}\right], e_{-\beta}\right) .
\end{aligned}
$$

Так как $\delta_{0}, \alpha \notin R_{0}, L_{ \pm 2}=0$, то $\left[e_{-\alpha}, e_{-\delta_{0}}\right] \in M_{0}$. Поэтому

$$
\psi\left(\left[e_{-\alpha}, e_{-\delta_{0}}\right], e_{-\beta}\right) \in\left\langle h_{0}\right\rangle_{K}
$$

по предположению индукции. Следовательно, для

$$
\psi\left(e_{-\gamma_{0}}, e_{-\delta_{0}}\right)=-\psi\left(\left[e_{-\alpha}, e_{-\delta_{0}}\right], e_{-\beta}\right)
$$

это также выполняется. Предложение доказано.

ПРЕДЛОЖЕНИЕ 11. Пусть $\mu \in \Lambda_{2}(R), \mu=\gamma_{0}+\delta_{0}$, əде $\left\langle\gamma_{0}, \delta_{0}\right\rangle=0$. Тогда

(1) если $L$ имеет тuп $A_{3}$ или $D_{l}, l \equiv 1(2)$, mо $H_{\mu}^{2}(L, L)=0$;

(2) если $L$ имеeт mun $D_{l}, l \equiv 0(2)$, mo $\operatorname{dim} H_{\mu}^{2}(L, L)=1$.

ДокАЗАТЕЛЬСтво. Пусть $\psi \in H_{\mu}^{2}(L, L), h \in \mathscr{H}, e_{-\gamma} \in L$. По лемме 3 корни являются ненулевыми функциями на $\mathscr{H}$, следовательно, $-\gamma+\mu \neq 0$, и если $-\gamma+\mu$ не является корнем, то $\psi\left(h, e_{-\gamma}\right)=0$. Предположим, что $-\gamma+\mu=\delta \in R$. Согласно лемме $3 \gamma+\delta=\mu$ не является корнем и $\left[e_{-\gamma}, e_{-\delta}\right]=0$. Тогда из леммы 5 следует, что

$$
\begin{aligned}
0 & =d \psi\left(e_{-\gamma}, e_{-\delta}, h\right)=\left[\psi\left(e_{-\gamma}, h\right), e_{-\delta}\right]+\left[\psi\left(e_{-\delta}, h\right), e_{-\gamma}\right] \\
& =\widehat{\psi}\left(e_{-\gamma}, h\right)\left[e_{\delta}, e_{-\delta}\right]+\widehat{\psi}\left(e_{-\delta}, h\right)\left[e_{\gamma}, e_{-\gamma}\right]=\widehat{\psi}\left(e_{-\gamma}, h\right) h_{\delta}+\widehat{\psi}\left(e_{-\delta}, h\right) h_{\gamma}
\end{aligned}
$$

Согласно следствию 2 леммы 3 и лемме $4 h_{\delta} \neq 0, h_{\gamma} \neq 0$ и $h_{\delta}+h_{\gamma} \neq 0$. Поэтому из формулы (5) получаем, что $\widehat{\psi}\left(e_{-\gamma}, h\right)=\widehat{\psi}\left(e_{-\delta}, h\right)=0$. Таким образом, $\psi(h, x)=0$ для всех $x \in L, h \in \mathscr{H}$. Утверждение (1) следует теперь из предложения 10.

Пусть $L$ - алгебра Ли типа $D_{l}, l \equiv 0(2)$. Согласно утверждению (2) предложения $10 \psi\left(e_{-\gamma}, e_{-\delta}\right)$ может быть отлично от нуля только при $\gamma+\delta=\mu$, и если $\mu=\gamma+\delta$, то $\psi\left(e_{-\gamma}, e_{-\delta}\right)=N h_{0}$, где $N \in K, h_{0} \in Z(L)$.

Пусть $\mu=\gamma+\delta=\gamma_{1}+\delta_{1}$. Согласно леммам 11 и $1-\gamma+\gamma_{1}$ является корнем, а $-\delta-\gamma_{1}$ не является корнем. Тогда так как $\left[h_{0}, L\right]=0$, то

$$
\begin{aligned}
0 & =d \psi\left(e_{-\gamma+\gamma_{1}}, e_{-\delta}, e_{-\gamma_{1}}\right)=\psi\left(\left[e_{-\gamma+\gamma_{1}}, e_{-\delta}\right], e_{-\gamma_{1}}\right)+\psi\left(\left[e_{-\gamma+\gamma_{1}}, e_{-\gamma_{1}}\right], e_{-\delta}\right) \\
& =\psi\left(e_{-\gamma+\gamma_{1}-\delta}, e_{-\gamma_{1}}\right)+\psi\left(e_{-\gamma+\gamma_{1}-\gamma_{1}}, e_{-\delta}\right)=\psi\left(e_{-\delta_{1}}, e_{-\gamma_{1}}\right)+\psi\left(e_{-\gamma}, e_{-\delta}\right) .
\end{aligned}
$$


Отсюда $\psi\left(e_{-\delta_{1}}, e_{-\gamma_{1}}\right)=\psi\left(e_{-\gamma}, e_{-\delta}\right)$. Таким образом, коэффициент $N$ не зависит от $\gamma, \delta$ и $\operatorname{dim} H_{\mu}^{2}(L, L) \leqslant \operatorname{dim} Z(L)=2(\operatorname{dim} Z(L)=2$ согласно предложению 1$)$.

Покажем, что для любого $z \in Z(L)$ коцепь $\chi_{z}=\sum_{\gamma+\delta=\mu} e_{-\gamma}^{*} \wedge e_{-\delta}^{*} \otimes z$ является коциклом.

Пусть $e_{-\gamma_{1}}, e_{-\gamma_{2}}, e_{-\gamma_{3}} \in L$. Если $\gamma_{1}+\gamma_{2}+\gamma_{3} \neq \mu$, то $d \chi_{z}\left(e_{-\gamma_{1}}, e_{-\gamma_{2}}, e_{-\gamma_{3}}\right)=0$. Предположим, что $\gamma_{1}+\gamma_{2}+\gamma_{3}=\mu$. Согласно лемме 12 можно считать, что $\gamma_{1}+\gamma_{2}$, $\gamma_{1}+\gamma_{3}$ являются корнями, а $\gamma_{2}+\gamma_{3}$ не является корнем. Тогда

$$
\begin{aligned}
d \chi_{z}\left(e_{-\gamma_{1}}, e_{-\gamma_{2}}, e_{-\gamma_{3}}\right)= & \chi_{z}\left(\left[e_{-\gamma_{1}}, e_{-\gamma_{2}}\right], e_{-\gamma_{3}}\right)+\chi_{z}\left(\left[e_{-\gamma_{1}}, e_{-\gamma_{3}}\right], e_{-\gamma_{2}}\right) \\
& +\chi\left(\left[e_{-\gamma_{2}}, e_{-\gamma_{3}}\right], e_{-\gamma_{1}}\right) \\
= & \chi_{z}\left(e_{-\gamma_{1}-\gamma_{2}}, e_{-\gamma_{3}}\right)+\chi_{z}\left(e_{-\gamma_{1}-\gamma_{3}}, e_{-\gamma_{2}}\right)=h_{0}+h_{0}=0 .
\end{aligned}
$$

Таким образом, $d \chi_{z}=0$ и $\chi_{z} \in Z_{\mu}^{2}(L, L)$.

Так как $\mu=\gamma_{0}+\delta_{0} \in \Lambda_{2}(R)$, то $h_{\gamma_{0}}+h_{\delta_{0}} \in Z(L)$. Положим $z_{1}=h_{\gamma_{0}}+h_{\delta_{0}}$. Тогда $Z(L)=\left\langle z_{1}, z_{2}\right\rangle_{K}$ для некоторого $z_{2} \in \mathscr{H}$.

Если $\gamma+\delta=\gamma_{0}+\delta_{0}$, то согласно леммам 11 и $1 \gamma-\gamma_{0}=\delta_{0}-\delta \in R$. Согласно следствию леммы $2 h_{\gamma-\gamma_{0}}=h_{\gamma}+h_{\gamma_{0}}, h_{\delta_{0}-\delta}=h_{\delta_{0}}+h_{\delta}$. Следовательно, $h_{\gamma}+h_{\delta}=h_{\gamma_{0}}+h_{\delta_{0}}=z_{1}$. Так как $C_{\mu}^{1}(L, L)=\left\langle e_{-\gamma}^{*} \otimes e_{\delta} \mid \gamma+\delta=\mu\right\rangle_{K}$, то для любого $\varphi \in C_{\mu}^{1}(L, L)$ и любых $\gamma, \delta$ таких, что $\gamma+\delta=\mu, d \varphi\left(e_{-\gamma}, e_{-\delta}\right) \in\left\langle z_{1}\right\rangle_{K}$. Поэтому $\chi_{z_{2}} \notin B_{\mu}^{2}(L, L)$.

Пусть $\varphi=\sum_{\gamma+\delta=\mu} e_{-\gamma}^{*} \otimes e_{\delta}$. Покажем, что $\chi_{z_{1}}=d \varphi$. Если $-\gamma-\delta+\mu \notin R \cup\{0\}$, то $d \varphi\left(e_{-\gamma}, e_{-\delta}\right)=0$. Если $-\gamma-\delta+\mu=0$, то $d \varphi\left(e_{-\gamma}, e_{-\delta}\right)=\left[\varphi\left(e_{-\gamma}\right), e_{-\delta}\right]+$ $\left[e_{-\gamma}, \varphi\left(e_{-\delta}\right)\right]=\left[e_{\delta}, e_{-\delta}\right]+\left[e_{\gamma}, e_{-\gamma}\right]=h_{\delta}+h_{\gamma}=z_{1}=\chi_{z_{1}}\left(e_{-\gamma}, e_{-\delta}\right)$. Пусть $-\gamma-\delta+\mu=\sigma \in R$. Тогда $\mu=\gamma+\delta+\sigma$. Если $\gamma+\delta \in R$, то, используя лемму 12 , можем считать, что $\gamma+\sigma \in R, \delta+\sigma \notin R$. Тогда $d \varphi\left(e_{-\gamma}, e_{-\delta}\right)=\varphi\left(\left[e_{-\gamma}, e_{-\delta}\right]\right)+$ $\left[\varphi\left(e_{-\delta}\right), e_{-\gamma}\right]=e_{\sigma}+e_{\sigma}=0$. Если $\gamma+\delta \notin R$, то из леммы 12 следует, что $\gamma+\sigma \in R$, $\delta+\sigma \in R$. Тогда $d \varphi\left(e_{-\gamma}, e_{-\delta}\right)=\left[\varphi\left(e_{-\gamma}\right), e_{-\delta}\right]+\left[\varphi\left(e_{-\delta}\right), e_{-\gamma}\right]=\left[e_{\delta+\sigma}, e_{-\delta}\right]+$ $\left[e_{\gamma+\sigma}, e_{-\gamma}\right]=e_{\sigma}+e_{\sigma}=0$. Следовательно, $\chi_{z_{1}}=d \varphi \in B^{2}(L, L)$.

Таким образом, $H_{\mu}^{2}(L, L)=\left\langle\chi_{z_{2}}\right\rangle_{K}$ и утверждение (2) доказано.

ЛЕмма 16. Пусть $L$ имеет тип $\bar{A}_{3}$ или $\bar{D}_{l}, \mu \in \Lambda_{2}(R), \mu=\gamma_{0}+\delta_{0}$, әде $\left\langle\gamma_{0}, \delta_{0}\right\rangle=0$. Если $\psi \in H_{\mu}^{2}(L, L)$, то $\widehat{\psi}\left(h, e_{-\gamma}\right)=\widehat{\psi}\left(h, e_{-\gamma_{1}}\right)$ для любых $h \in \mathscr{H}$, $\gamma, \gamma_{1} \in R$ mаких, что $-\gamma+\mu \in R,-\gamma_{1}+\mu \in R$.

ДокАЗАТЕЛЬСТвО. Пусть $-\gamma+\mu=\delta,-\gamma_{1}+\mu=\delta_{1}$. Из леммы 5 следует, что

$$
\begin{aligned}
0 & =d \psi\left(e_{-\gamma}, e_{-\gamma_{1}}, h\right)=\left[\psi\left(e_{-\gamma}, h\right), e_{-\gamma_{1}}\right]+\left[\psi\left(e_{-\gamma_{1}}, h\right), e_{-\gamma}\right]+\psi\left(\left[e_{-\gamma}, e_{-\gamma_{1}}\right], h\right) \\
& =\widehat{\psi}\left(e_{-\gamma}, h\right)\left[e_{\delta}, e_{-\gamma_{1}}\right]+\widehat{\psi}\left(e_{-\gamma_{1}}, h\right)\left[e_{\delta_{1}}, e_{-\gamma}\right]+\psi\left(\left[e_{-\gamma}, e_{-\gamma_{1}}\right], h\right) .
\end{aligned}
$$

Предположим, что $\left\{\gamma_{1}, \delta_{1}\right\} \neq\{\gamma, \delta\}$. Так как $\mu=\gamma+\delta=\gamma_{1}+\delta_{1}$, то из лемм 11 и 1 следует, что $\gamma+\gamma_{1}$ не является корнем, а $\delta-\gamma_{1}=\delta_{1}-\gamma$ является корнем. Тогда $\psi\left(\left[e_{-\gamma}, e_{-\gamma_{1}}\right], h\right)=0,\left[e_{\delta}, e_{-\gamma_{1}}\right]=\left[e_{\delta_{1}}, e_{-\gamma}\right] \neq 0$ и из равенства (6) следует, что $\widehat{\psi}\left(e_{-\gamma}, h\right)=\widehat{\psi}\left(e_{-\gamma_{1}}, h\right)$.

Если $\gamma_{1}=\delta$, то $\gamma+\gamma_{1}=\mu \notin R$ и из равенства (6) следует, что $\widehat{\psi}\left(e_{-\gamma}, h\right) h_{\delta}+$ $\widehat{\psi}\left(e_{-\delta}, h\right) h_{\gamma}=0$. Так как $h_{\gamma} \neq 0$ и $h_{\delta} \neq 0$ (следствие 2 леммы 3$)$, то $\widehat{\psi}\left(e_{-\gamma}, h\right)=$ $\widehat{\psi}\left(e_{-\delta}, h\right)$. 
ПРЕДЛОЖЕНИЕ 12. Пусть L имеeт mun $\bar{D}_{l}, \mu \in \Lambda_{2}(R), \mu=\gamma_{0}+\delta_{0}$, əде $\left\langle\gamma_{0}, \delta_{0}\right\rangle=0$. Тогда

(1) eсли $l \equiv 0(2)$, mо $H_{\mu}^{2}(L, L)=0$;

(2) если $l \equiv 1(2)$, mo $\operatorname{dim} H_{\mu}^{2}(L, L) \leqslant 1$.

ДоКАЗАТЕЛЬСтво. Пусть $\psi \in H_{\mu}^{2}(L, L)$. Используя лемму 13 , можем считать, что $\mu=\alpha_{1}+\alpha_{l}$. Тогда $\alpha_{l} \pm \alpha_{i}, \pm \alpha_{i}-\alpha_{l}+\mu$ не являются корнями при $i=3, \ldots, l-1$ И

$$
0=d \psi\left(e_{\alpha_{i}}, e_{-\alpha_{i}}, e_{-\alpha_{l}}\right)=\psi\left(\left[e_{\alpha_{i}}, e_{-\alpha_{i}}\right], e_{-\alpha_{l}}\right)=\psi\left(h_{i}, e_{-\alpha_{l}}\right) .
$$

Пусть $l \equiv 0(2)$. Так как $\pm \alpha_{2}+\left(-\alpha_{1}-\alpha_{2}-\alpha_{l}\right),-\alpha_{2}+\left(-\alpha_{1}-\alpha_{2}-\alpha_{l}\right)+\mu$ не являются корнями, то

$$
\begin{aligned}
& d \psi\left(e_{\alpha_{2}}, e_{-\alpha_{2}}, e_{-\alpha_{1}-\alpha_{2}-\alpha_{l}}\right) \\
& \quad=\psi\left(\left[e_{\alpha_{2}}, e_{-\alpha_{2}}\right], e_{-\alpha_{1}-\alpha_{2}-\alpha_{l}}\right)+\left[\psi\left(e_{\alpha_{2}}, e_{-\alpha_{1}-\alpha_{2}-\alpha_{l}}\right), e_{-\alpha_{2}}\right] .
\end{aligned}
$$

Так как $\alpha_{2}+\left(-\alpha_{1}-\alpha_{2}-\alpha_{l}\right)=-\mu$, то из леммы 15 следует, что

$$
\psi\left(e_{\alpha_{2}}, e_{-\alpha_{1}-\alpha_{2}-\alpha_{l}}\right)=N_{1} h_{2}+N_{2} h_{c}, \quad N_{1}, N_{2} \in K, \quad h_{c} \in Z(L) .
$$

Поэтому $\left[\psi\left(e_{\alpha_{2}}, e_{-\alpha_{1}-\alpha_{2}-\alpha_{l}}\right), e_{-\alpha_{2}}\right]=0$ и

$$
0=d \psi\left(e_{\alpha_{2}}, e_{-\alpha_{2}}, e_{-\alpha_{1}-\alpha_{2}-\alpha_{l}}\right)=\psi\left(h_{2}, e_{-\alpha_{1}-\alpha_{2}-\alpha_{l}}\right) .
$$

Используя лемму 16 , из равенств $(7)$ и (8) получаем, что $\psi\left(h_{i}, x\right)=0$ для любого $i=2, \ldots, l-1$ и любого $x \in L$. Из предложения 1 следует, что $\left\langle h_{\alpha_{2}}, \ldots, h_{l-1}\right\rangle=\mathscr{H}$. Таким образом, $\psi(h, x)=0$ для всех $h \in \mathscr{H}, x \in L$. Теперь утверждение (1) следует из предложения 10 .

Пусть $l \equiv 1(2)$. Из леммы 16 и предложения 10 следует, что коцикл $\psi$ может быть не когомологичен нулю, только если существует $h \in \mathscr{H}$ такой, что $\psi(h, x) \neq 0$, и размерность $H_{\mu}^{2}(L, L)$ не превосходит числа таких линейно независимых $h$.

Из равенства (7) и леммы 16 следует, что $\psi\left(h_{i}, x\right)=0$ для всех $i=3, \ldots, l-1$ и любого $x \in L$. Так как $h_{1}+h_{l}=0$ в алгебре типа $\bar{D}_{l}$, то $\psi\left(x, h_{1}\right)=\psi\left(x, h_{l}\right)$ для любого $x \in L$. Остается выяснить, как связаны $\psi\left(x, h_{1}\right)$ и $\psi\left(x, h_{2}\right)$.

Так как $\alpha_{2} \pm\left(\alpha_{1}+\alpha_{2}+\alpha_{3}\right), \alpha_{2} \pm\left(\alpha_{1}+\alpha_{2}+\alpha_{3}\right)+\mu$ не являются корнями, то

$$
\begin{aligned}
0 & =d \psi\left(e_{\alpha_{2}}, e_{\alpha_{1}+\alpha_{2}+\alpha_{3}}, e_{-\alpha_{1}-\alpha_{2}-\alpha_{3}}\right)=\psi\left(e_{\alpha_{2}},\left[e_{\alpha_{1}+\alpha_{2}+\alpha_{3}}, e_{-\alpha_{1}-\alpha_{2}-\alpha_{3}}\right]\right) \\
& =\psi\left(e_{\alpha_{2}}, h_{1}+h_{2}+h_{3}\right)=\psi\left(e_{\alpha_{2}}, h_{1}\right)+\psi\left(e_{\alpha_{2}}, h_{2}\right) .
\end{aligned}
$$

Следовательно, $\psi\left(e_{\alpha_{2}}, h_{1}\right)=\psi\left(e_{\alpha_{2}}, h_{2}\right)$. Тогда из леммы 16 получаем, что

$$
\psi\left(x, h_{1}\right)=\psi\left(x, h_{2}\right)
$$

для любого $x \in L$. Таким образом, $\operatorname{dim} H_{\mu}^{2}(L, L) \leqslant 1$.

Чтобы описать когомологии алгебр Ли типа $\bar{D}_{l}$ при нечетном $l$ и алгебры Ли типа $\bar{A}_{3}$, нам понадобится следуюшее описание $\bar{D}_{l}$ и $\bar{A}_{3}$. 
Можно считать, что $\bar{A}_{3}=\bar{D}_{3}$. Согласно [8] группа автоморфизмов алгебры Ли $L$ типа $\bar{D}_{l}$ при $l$ нечетном изоморфна групше Шевалле типа $C_{l}$. Пусть $V$ - векторное пространство над $K$ размерности $2 l$ с симплектической формой $(\cdot, \cdot)$. Группа $G=$ Aut $L$ является симплектической группой $\operatorname{Sp}(2 l)$, ассоциированной с формой $(\cdot, \cdot)$. Выберем симплектический базис $\left\{e_{1}, \ldots, e_{l}, e_{-l}, \ldots, e_{-1}\right\}$ в $V$, состоящий из собственных векторов относительно действия максимального тора группы $G$. Пусть $e_{i}$ имеет вес $\varepsilon_{i}, e_{-i}$ имеет вес $\left(-\varepsilon_{i}\right), i=1, \ldots, l$. Алгебру Ли $\widetilde{L}$ типа $C_{l}$ отождествим с $S^{(2)} V$ (где $S^{(2)} V$ - нулевая компонента гамильтоновой алгебры Ли $H(V)$ в разделенных степенях относительно стандартной градуировки, см. [13]). Факторалгебра внешней алгебры $\wedge^{2} V$ по идеалу $I=\left\langle e_{1} e_{-1}+\cdots+e_{l} e_{-l}\right\rangle$ является идеалом в $\widetilde{L} / I$, изоморфным алгебре Ли $L$ (это верно только при нечетном $l$ ).

Teорема 1. Пусть L имеет тип $\bar{D}_{l}, l>3$ нечетное. Тогда $\operatorname{dim} H^{2}(L, L)=$ $2 l$ и с точностью до морфизма Фробениуса $H^{2}(L, L)$ как модуль над $\operatorname{Sp}(2 l)=$ Aut $G$ изоморфен $V$, где $V$ - стандартный модуль над $\operatorname{Sp}(2 l)$.

ДокАЗАТЕЛЬство. Из результатов $\S 3$ следует, что $H_{0}^{2}(L, L)=0$. Из предложений 7-9 и леммы 13 получаем, что $H^{2}(L, L)=\bigoplus_{\mu \in M} H_{\mu}^{2}(L, L)$, где $M=$ $\left\{ \pm\left(\alpha_{1}-\alpha_{l}\right), \pm\left(\alpha_{1}+\alpha_{l}\right), \pm\left(\alpha_{1}+\alpha_{l}+2 \alpha_{2}\right), \ldots, \pm\left(\alpha_{1}+\alpha_{l}+2 \alpha_{2}+\cdots+2 \alpha_{l-1}\right)\right\}$. Из предложения 12 тогда следует, что $\operatorname{dim} H^{2}(L, L) \leqslant 2 l$.

Построим гомоморфизм модуля $V$ в модуль $H^{2}(L, L)$.

Определим $\Phi_{0}: V \rightarrow C^{2}(L, L)$. Пусть $\left\{w_{1} w_{2}, v\right\}=\left(w_{1}, v\right) w_{2}+\left(w_{2}, v\right) w_{1}$, $\left\{v_{1} v_{2}, v_{3} v_{4}\right\}=\left(v_{1}, v_{3}\right) v_{2} v_{4}+\left(v_{1}, v_{4}\right) v_{2} v_{3}+\left(v_{2}, v_{3}\right) v_{1} v_{4}+\left(v_{2}, v_{4}\right) v_{1} v_{3}-$ скобки Пуассона в гамильтоновой алгебре $H(V)$. Для любого $v \in V$ положим $\Phi_{0}(v)=\psi$, где

$$
\psi\left(w_{1} w_{2}, w_{3} w_{4}\right)=\left\{w_{1} w_{2}, v\right\}\left\{w_{3} w_{4}, v\right\}+v\left\{v,\left(w_{3}, w_{4}\right) w_{1} w_{2}+\left(w_{1}, w_{2}\right) w_{3} w_{4}\right\} .
$$

Отметим, что $\psi$ корректно определено на элементах $\wedge^{2} V / I \cong L$, также $\psi$ линейно и кососимметрично, а следовательно, является элементом $C^{2}(L, L)$.

Вычис ление $d \psi$ на произвольных элементах $w_{1} w_{2}, w_{3} w_{4}, w_{5} w_{6}$ показывает, что $\psi \in Z^{2}(L, L)$. Рассмотрим, например, слагаемое

$$
\left\{\psi\left(w_{1} w_{2}, w_{3} w_{4}\right), w_{5} w_{6}\right\}=\left\{\left(v, w_{1}\right)\left(v, w_{3}\right) w_{2} w_{4}+\cdots, w_{5} w_{6}\right\}
$$

в $d \psi\left(w_{1} w_{2}, w_{3} w_{4}, w_{5} w_{6}\right)$. Здесь есть член $\left(v, w_{1}\right)\left(v, w_{3}\right)\left(w_{2}, w_{5}\right) w_{4} w_{6}$. Но точно такой же член возникнет в слагаемом

$$
\begin{aligned}
\psi\left(\left\{w_{1} w_{2}, w_{5} w_{6}\right\}, w_{3} w_{4}\right) & =\psi\left(\left(w_{2}, w_{5}\right) w_{1} w_{6}+\cdots, w_{3} w_{4}\right) \\
& =\left(w_{2}, w_{5}\right)\left(v, w_{1}\right)\left(v, w_{3}\right) w_{6} w_{4}+\cdots
\end{aligned}
$$

Следовательно, в $d \psi$ эти члены взаимно уничтожатся. Аналогично сокращаются остальные слагаемые в $d \psi$.

Пусть $\left\{e_{1}, \ldots, e_{l}, e_{-l}, \ldots, e_{-1}\right\}$ - симплектический базис в $V$. Рассмотрим коцикл $\Phi_{0}\left(e_{i}\right)$.

Пусть $j \neq \pm i, k \neq \pm i$. Тогда $\Phi_{0}\left(e_{i}\right)\left(e_{k} e_{-k}, e_{-i} e_{j}\right)=\left(e_{k}, e_{-k}\right)\left(e_{i}, e_{-i}\right) e_{i} e_{j}=$ $e_{i} e_{j} \neq 0$. Поэтому $\Phi_{0}\left(e_{i}\right)(h, x) \neq 0$ для некоторых $h \in \mathscr{H}, x \in L$. 
Для $\varphi \in C_{\mu}^{1}(L, L), h \in \mathscr{H}, e_{\gamma} \in L$ выполняется $d \varphi\left(h, e_{\gamma}\right)=\left[e_{\gamma}, \varphi(h)\right]$. Поэтому $d \varphi\left(h, e_{\gamma}\right) \neq 0$ только при условии $\varphi(h) \neq 0$, а следовательно, при условии $\mu \in$ $R \cup\{0\}$. Так как коцикл $\Phi_{0}\left(e_{i}\right)$ имеет вес $2 \varepsilon_{i}$ и $2 \varepsilon_{i} \notin R \cup\{0\}$, то $\Phi_{0}\left(e_{i}\right)$ не является кограницей. Таким образом, $\Phi_{0}\left(e_{ \pm 1}\right), \ldots, \Phi_{0}\left(e_{ \pm l}\right)$ определяют $2 l$ нетривиальных коциклов различных весов.

Поскольку $\Phi_{0}(v) \in Z^{2}(L, L)$ для любого $v \in V$, то $\Phi_{0}\left(e_{i}+e_{j}\right)-\Phi_{0}\left(e_{i}\right)-$ $\Phi_{0}\left(e_{j}\right) \in Z_{\varepsilon_{i}+\varepsilon_{j}}^{2}(L, L)=B_{\varepsilon_{i}+\varepsilon_{j}}^{2}(L, L) \quad\left(H^{2}(L, L)=\bigoplus_{k=-l}^{l} H_{2 \varepsilon_{k}}^{2}(L, L)\right)$. Следовательно, $\Phi_{0}$ индуцирует аддитивное отображение $\Phi: V \rightarrow H^{2}(L, L)$. Так как $\operatorname{dim} H^{2}(L, L) \leqslant 2 l$, то $\Phi$ является биекцией.

Легко проверить, что

$$
\Phi(g v)\left(w_{1} w_{2}, w_{3} w_{4}\right)=g\left(\Phi(v)\left(g^{-1} w_{1} g^{-1} w_{2}, g^{-1} w_{3} g^{-1} w_{4}\right)\right)
$$

для любого $g \in \operatorname{Sp}(2 l)$ (так как $\psi$ линейно). Из определений действия $\operatorname{Aut} L=$ $\mathrm{Sp}(2 l)$ на $V$ и на $C^{2}(L, L)$ сразу следует, что $\Phi$ перестановочно с действием Aut $L$. Так как для любого $k \in K \Phi(k v)=k^{2} \Phi(v)$, то действие $\mathrm{Sp}(2 l)$ на $H^{2}(L, L)$ отличается от действия на $V$ морфизмом $\Phi$ робениуса.

В алгебре Ли типа $\bar{A}_{3}$ ситуация аналогична.

Tеорема 2. Пусть $L$ - алгебра Ли типа $\bar{A}_{3}, V$ - стандартный шестимерный модуль над $\mathrm{Sp}(6) \cong \mathrm{Aut} L$. Тогда $\operatorname{dim} H^{2}(L, L)=20$ и представление $\mathrm{Sp}(6)$ на $H^{2}(L, L)$ әквивалентно композиции морфизма Фробениуса и естественного представления на $\wedge^{3} \mathrm{~V}$.

ДоказАтельство. Пусть $\left\{\alpha_{1}, \alpha_{2}, \alpha_{3}\right\}$ - базис системы корней типа $A_{3}$.

В $\S 3$ доказано, что $H_{0}^{2}(L, L)=0$. Из предложений 7,8 следует, что

$$
H^{2}(L, L)=\bigoplus_{\mu \in \Lambda_{2}(R) \cap\left(M_{2} \cup M_{3}\right)} H_{\mu}^{2}(L, L),
$$

где $M_{2}=\{\mu=\alpha+\beta+\gamma \mid\langle\alpha, \beta\rangle=\langle\alpha, \gamma\rangle=\langle\beta, \gamma\rangle=1\}, M_{3}=\{\mu=\gamma+\delta \mid\langle\gamma, \delta\rangle=0\}$. Согласно утверждению (2) предложения $9 \operatorname{dim} H_{\mu}^{2}(L, L)=1$, если $\mu \in M_{2} \cap$ $\Lambda_{2}(R)$. Также из доказательства предложения 9 получаем, что $M_{2} \cap \Lambda_{2}(R)=$ $\left\{ \pm\left(3 \alpha_{1}+2 \alpha_{2}+\alpha_{3}\right), \pm\left(\alpha_{1}+2 \alpha_{2}+3 \alpha_{3}\right), \pm\left(-\alpha_{1}+2 \alpha_{2}+\alpha_{3}\right), \pm\left(\alpha_{1}+2 \alpha_{2}-\alpha_{3}\right)\right\}$. Все элементы этого множества сопряжены относительно $W(R)$. Если $\mu \in M_{3} \cap$ $\Lambda_{2}(R)$, то из леммы 16 и предложения 10 следует, что $\operatorname{dim} H_{\mu}^{2}(L, L) \leqslant \operatorname{dim} \mathscr{H}=2$. Из доказательства предложения 10 получаем, что $M_{3} \cap \Lambda_{2}(R)=\left\{ \pm\left(\alpha_{1}+\alpha_{3}\right)\right.$, $\left.\pm\left(\alpha_{1}-\alpha_{3}\right), \pm\left(\alpha_{1}+2 \alpha_{2}+\alpha_{3}\right)\right\}$ и все элементы $M_{3}$ сопряжены относительно $W(R)$. Таким образом, $\operatorname{dim} H^{2}(L, L) \leqslant 8+2 \cdot 6=20$.

Построим гомоморфизм модуля $\wedge^{3} V$ в модуль $H^{2}(L, L)$.

Пусть $\left\{v_{1} v_{2}, v\right\}=\left(v_{1}, v\right) v_{2}+\left(v_{2}, v\right) v_{1},\left\{v_{1} v_{2} v_{3}, v\right\}=\left(v_{1}, v\right) v_{2} v_{3}+\left(v_{2}, v\right) v_{1} v_{3}+$ $\left(v_{3}, v\right) v_{1} v_{2}-$ скобки Пуассона.

Для $w_{1} w_{2}, w_{3} w_{4} \in \wedge^{2} V$ положим

$$
\begin{aligned}
\Delta\left(w_{1} w_{2}, w_{3} w_{4}\right) & =\left(w_{3}, w_{4}\right) w_{1} w_{2}+\left(w_{1}, w_{2}\right) w_{3} w_{4}, \\
v \circ w_{1} w_{2} & =\left(v, w_{1}\right) v w_{2}+\left(v, w_{2}\right) v w_{1}, \\
\Gamma_{v_{1} w_{2} w_{3}}^{w_{1} w_{3}} & =\left|\begin{array}{lll}
\left(v_{1}, w_{1}\right) & \left(v_{2}, w_{1}\right) & \left(v_{3}, w_{1}\right) \\
\left(v_{1}, w_{2}\right) & \left(v_{2}, w_{2}\right) & \left(v_{3}, w_{2}\right) \\
\left(v_{1}, w_{3}\right) & \left(v_{2}, w_{3}\right) & \left(v_{3}, w_{3}\right)
\end{array}\right| .
\end{aligned}
$$


Определим $\Phi_{0}: \wedge^{3} V \rightarrow C^{2}(L, L), \Phi_{0}\left(v_{1} v_{2} v_{3}\right)=\psi$, где

$$
\begin{aligned}
\psi\left(w_{1} w_{2}, w_{3} w_{4}\right)=\left\{\left\{v_{1} v_{2} v_{3}, w_{1}\right\}, w_{2}\right\}\left\{\left\{v_{1} v_{2} v_{3}, w_{3}\right\}, w_{4}\right\} \\
+\left(v_{2}, v_{3}\right)^{2} v_{1} \circ \Delta\left(w_{1} w_{2}, w_{3} w_{4}\right)+\left(v_{1}, v_{3}\right)^{2} v_{2} \circ \Delta\left(w_{1} w_{2}, w_{3} w_{4}\right) \\
+\left(v_{1}, v_{2}\right)^{2} v_{3} \circ \Delta\left(w_{1} w_{2}, w_{3} w_{4}\right) \\
+\left(\sum_{i=1}^{4} \Gamma_{v_{1} v_{2} v_{3}}^{w_{1} \cdots \widehat{w}_{i} \cdots w_{4}} w_{i}\right)\left(\left(v_{2}, v_{3}\right) v_{1}+\left(v_{1}, v_{3}\right) v_{2}+\left(v_{1}, v_{2}\right) v_{3}\right) .
\end{aligned}
$$

Прямая проверка показывает, что $\psi$ корректно определено на $\wedge^{2} V / I \cong L$ и $\psi \in Z^{2}(L, L)$.

Так как $B_{2 \varepsilon_{i}+2 \varepsilon_{j}+2 \varepsilon_{k}}^{2}(L, L)=0$ при $i \neq \pm j, k \neq \pm j, i \neq \pm k$, то $\Phi_{0}\left(e_{i} e_{j} e_{k}\right)$ определяют 8 нетривиальных коциклов различных весов.

Такое же рассуждение, как в доказательстве теоремы 1 , показывает, что любой коцикл, удовлетворяющий условию $\psi(h, x) \neq 0$ для некоторых $x \in L, h \in \mathscr{H}$, не когомологичен нулю. Так как $\Phi_{0}\left(e_{i} e_{j} e_{-j}\right)\left(e_{k} e_{-k}, e_{-i} e_{s}\right)=e_{i} e_{s} \neq 0$ при $k \neq \pm i$, $k \neq \pm j$, то $\Phi_{0}\left(e_{i} e_{j} e_{-j}\right)$ нетривиальны. Коциклы $\Phi_{0}\left(e_{i} e_{j} e_{-j}\right)$ и $\Phi_{0}\left(e_{i} e_{k} e_{-k}\right)$ при $k \neq \pm j$ не когомологичны. Например, $\Phi_{0}\left(e_{1} e_{2} e_{-2}\right)\left(e_{3} e_{-3}, e_{-1} e_{3}\right)=e_{1} e_{3}, \mathrm{a}$ $\Phi_{0}\left(e_{1} e_{3} e_{-3}\right)\left(e_{3} e_{-3}, e_{-1} e_{3}\right)=0$. Следовательно, $\Phi_{0}\left(e_{i} e_{j} e_{-j}\right)$ определяют 12 нетривиальных не когомологичных коциклов.

Таким образом, $\Phi_{0}$ определяет не меньше 20 нетривиальных не когомологичных коциклов.

Так же как в доказательстве теоремы 1 , нетрудно показать, что отображение $\Phi_{0}$ индуцирует аддитивное отображение $\Phi$ пространства $\wedge^{3} V$ на $H^{2}(L, L)$. Так как $\operatorname{dim} H^{2}(L, L) \leqslant 20$, то $\Phi$ является биекцией. Отображение $\Phi$ перестановочно с естественным действием $\operatorname{Sp}(6)$ на $\wedge^{3} V$ и $H^{2}(L, L)$. Для любого $k \in K$ $\Phi\left(k v_{1} v_{2} v_{3}\right)=k^{2} \Phi\left(v_{1} v_{2} v_{3}\right)$. Следовательно, $\Phi$ с точностью до морфизма Фробениуса является изоморфизмом $\mathrm{Sp}(6)$-модулей.

Осталось исследовать когомологии весов вида $\mu=\alpha+\beta+\gamma$, где $\langle\alpha, \beta\rangle=$ $\langle\alpha, \gamma\rangle=\langle\beta, \gamma\rangle=0$. Из предложения 7 следует, что такие веса могут возникать только у алгебры Ли одного из типов $A_{5}, D_{6}, E_{7}, \bar{A}_{5}, \bar{D}_{6}, \bar{E}_{7}$.

ПРЕДЛОЖЕНИЕ 13. Пусть $\mu \in \Lambda_{2}(R), \mu=\alpha+\beta+\gamma$, гдe $\langle\alpha, \beta\rangle=\langle\alpha, \gamma\rangle=$ $\langle\beta, \gamma\rangle=0$. Тогда

(1) если $L$ имеет muп $A_{5}, D_{6}$ или $E_{7}$, то $H_{\mu}^{2}(L, L)=0$;

(2) если $L$ имеет muп $\bar{A}_{5}, \bar{D}_{6}$ или $\bar{E}_{7}$, mo $\operatorname{dim} H_{\mu}^{2}(L, L)=1$.

ДокАЗАТЕЛЬСТво. Пусть $\psi \in H_{\mu}^{2}(L, L)$. Из утверждения (6) предложения 7 следует, что $B_{\mu}^{2}(L, L)=0$ и $\psi(h, x)=0$ для любых $h \in \mathscr{H}, x \in L$.

Предположим, что $\mu=\gamma_{1}+\gamma_{2}+\gamma_{3}$ для некоторых $\gamma_{1}, \gamma_{2}, \gamma_{3} \in R$. Согласно утверждению (6) предложения $7 \mu$ нельзя представить в виде суммы двух корней. Поэтому из утверждений (3), (4) предложения 7 следует, что $\left\langle\gamma_{1}, \gamma_{2}\right\rangle=\left\langle\gamma_{1}, \gamma_{3}\right\rangle=$ $\left\langle\gamma_{2}, \gamma_{3}\right\rangle=0,1$. В случае $\left\langle\gamma_{1}, \gamma_{2}\right\rangle=\left\langle\gamma_{1}, \gamma_{3}\right\rangle=\left\langle\gamma_{2}, \gamma_{3}\right\rangle=1$ из утверждения (5) предложения 7 получаем противоречие условию $\langle\alpha, \beta\rangle=\langle\alpha, \gamma\rangle=\langle\beta, \gamma\rangle=0$. 
Поэтому $\left\langle\gamma_{1}, \gamma_{2}\right\rangle=\left\langle\gamma_{1}, \gamma_{3}\right\rangle=\left\langle\gamma_{2}, \gamma_{3}\right\rangle=0$ для любых $\gamma_{1}, \gamma_{2}, \gamma_{3} \in R$ таких, что $\mu=\gamma_{1}+\gamma_{2}+\gamma_{3}$. Тогда для любого $\psi \in C_{\mu}^{2}(L, L)$

$$
\begin{aligned}
d \psi\left(e_{-\gamma_{1}}, e_{-\gamma_{2}}, e_{-\gamma_{3}}\right) & \\
= & {\left[\psi\left(e_{-\gamma_{1}}, e_{-\gamma_{2}}\right), e_{-\gamma_{3}}\right]+\left[\psi\left(e_{-\gamma_{1}}, e_{-\gamma_{3}}\right), e_{-\gamma_{2}}\right]+\left[\psi\left(e_{-\gamma_{3}}, e_{-\gamma_{2}}\right), e_{-\gamma_{1}}\right] } \\
= & \widehat{\psi}\left(e_{-\gamma_{1}}, e_{-\gamma_{2}}\right)\left[e_{\gamma_{3}}, e_{-\gamma_{3}}\right]+\widehat{\psi}\left(e_{-\gamma_{1}}, e_{-\gamma_{3}}\right)\left[e_{\gamma_{2}}, e_{-\gamma_{2}}\right] \\
& +\widehat{\psi}\left(e_{-\gamma_{3}}, e_{-\gamma_{2}}\right)\left[e_{\gamma_{1}}, e_{-\gamma_{1}}\right] \\
= & \widehat{\psi}\left(e_{-\gamma_{1}}, e_{-\gamma_{2}}\right) h_{\gamma_{3}}+\widehat{\psi}\left(e_{-\gamma_{1}}, e_{-\gamma_{3}}\right) h_{\gamma_{2}}+\widehat{\psi}\left(e_{-\gamma_{3}}, e_{-\gamma_{2}}\right) h_{\gamma_{1}} .
\end{aligned}
$$

Если $L$ имеет тип $A_{5}, D_{l}$ или $E_{7}$, то $h_{\gamma_{3}}, h_{\gamma_{2}}, h_{\gamma_{1}}$ линейно независимы (следствие 2 леммы 3 , лемма 4 ) и из равенства (9) следует, что

$$
\widehat{\psi}\left(e_{-\gamma_{1}}, e_{-\gamma_{2}}\right)=\widehat{\psi}\left(e_{-\gamma_{1}}, e_{-\gamma_{3}}\right)=\widehat{\psi}\left(e_{-\gamma_{3}}, e_{-\gamma_{2}}\right)=0
$$

для любых $\gamma_{1}, \gamma_{2}, \gamma_{3} \in R$ таких, что $\mu=\gamma_{1}+\gamma_{2}+\gamma_{3}$. Следовательно, $\psi=0$ и утверждение (1) доказано.

Так как в алгебрах типа $\bar{A}_{5}, \bar{D}_{6}, \bar{E}_{7}$ выполняется $h_{\gamma_{1}}+h_{\gamma_{2}}+h_{\gamma_{3}}=0$, то из следствия 2 леммы 3 и равенства (9) получаем, что $\widehat{\psi}\left(e_{-\gamma_{1}}, e_{-\gamma_{2}}\right)=\widehat{\psi}\left(e_{-\gamma_{1}}, e_{-\gamma_{3}}\right)=$ $\widehat{\psi}\left(e_{-\gamma_{3}}, e_{-\gamma_{2}}\right)$.

Покажем, что $\widehat{\psi}\left(e_{-\gamma_{1}}, e_{-\gamma_{2}}\right)=\widehat{\psi}\left(e_{-\alpha}, e_{-\beta}\right)$. Сначала рассмотрим случай, когда $A=\{\alpha, \beta, \gamma\} \cap\left\{\gamma_{1}, \gamma_{2}, \gamma_{3}\right\} \neq \varnothing$. Можем считать, что $\gamma_{1}=\alpha$.

Рассмотрим $d \psi\left(e_{-\gamma_{1}}, e_{-\gamma_{2}}, e_{-\gamma}\right)$. Так как $\alpha=\gamma_{1}$ и $\langle\alpha, \gamma\rangle=\left\langle\gamma_{1}, \gamma_{2}\right\rangle=0$, то $\psi\left(\left[e_{-\gamma_{1}}, e_{-\gamma_{2}}\right], e_{-\gamma}\right)=0$ и $\psi\left(\left[e_{-\gamma_{1}}, e_{-\gamma}\right], e_{-\gamma_{2}}\right)=0$. Из равенств $\langle\mu, \gamma\rangle=\langle\alpha+$ $\beta+\gamma, \gamma\rangle=\langle\gamma, \gamma\rangle=2$ и $\langle\mu, \gamma\rangle=\left\langle\alpha+\gamma_{2}+\gamma_{3}, \gamma\right\rangle=\left\langle\gamma_{2}+\gamma_{3}, \gamma\right\rangle$ следует, что $\left\langle\gamma_{2}, \gamma\right\rangle+\left\langle\gamma_{3}, \gamma\right\rangle=2$. Если $\gamma_{2}=\gamma$ или $\gamma_{3}=\gamma$, то $\{\alpha, \beta, \gamma\}=\left\{\gamma_{1}, \gamma_{2}, \gamma_{3}\right\}$ и $\widehat{\psi}\left(e_{-\gamma_{1}}, e_{-\gamma_{2}}\right)=\widehat{\psi}\left(e_{-\alpha}, e_{-\beta}\right)$. Если $\gamma_{2} \neq \gamma, \gamma_{3} \neq \gamma$, то $\left\langle\gamma_{2}, \gamma\right\rangle \neq 2$ и $\left\langle\gamma_{3}, \gamma\right\rangle \neq 2$. Поэтому из равенства $\left\langle\gamma_{2}, \gamma\right\rangle+\left\langle\gamma_{3}, \gamma\right\rangle=2$ следует, что $\left\langle\gamma_{2}, \gamma\right\rangle=\left\langle\gamma_{3}, \gamma\right\rangle=1$. Тогда согласно лемме $1-\gamma_{2}-\gamma \notin R, \gamma_{3}-\gamma \in R$. Поэтому $\psi\left(\left[e_{-\gamma_{2}}, e_{-\gamma}\right], e_{-\gamma_{1}}\right)=0$. Из условий $\left\langle\gamma_{1}, \gamma\right\rangle=\langle\alpha, \gamma\rangle=0,\left\langle\gamma_{1}, \gamma_{3}\right\rangle=0$ следует, что $\left\langle\gamma_{1}, \gamma_{3}-\gamma\right\rangle=0$. Поэтому так как $\gamma_{3}-\gamma \in R$, то $-\gamma_{2}-\gamma+\mu=\gamma_{1}+\gamma_{3}-\gamma \notin R$ и $\psi\left(e_{-\gamma_{2}}, e_{-\gamma}\right)=0$.

Таким образом,

$$
0=d \psi\left(e_{-\gamma_{1}}, e_{-\gamma_{2}}, e_{-\gamma}\right)=\left[\psi\left(e_{-\gamma_{1}}, e_{-\gamma_{2}}\right), e_{-\gamma}\right]+\left[\psi\left(e_{-\gamma_{1}}, e_{-\gamma}\right), e_{-\gamma_{2}}\right] .
$$

Так как $\left\langle\gamma_{2}, \gamma_{3}-\gamma\right\rangle=-\left\langle\gamma_{2}, \gamma\right\rangle=-1$ и $\gamma_{3}-\gamma \in R$, то по лемме $1-\gamma_{1}-\gamma+$ $\mu=\gamma_{2}+\gamma_{3}-\gamma \in R$. Поэтому из последнего равенства следует, что $\widehat{\psi}\left(e_{-\gamma_{1}}, e_{-\gamma_{2}}\right)=$ $\widehat{\psi}\left(e_{-\gamma_{1}}, e_{-\gamma}\right)$. С учетом $\alpha=\gamma_{1}$ получаем, что $\widehat{\psi}\left(e_{-\gamma_{1}}, e_{-\gamma_{2}}\right)=\widehat{\psi}\left(e_{-\alpha}, e_{-\gamma}\right)=$ $\widehat{\psi}\left(e_{-\alpha}, e_{-\beta}\right)$.

Пусть $A=\{\alpha, \beta, \gamma\} \cap\left\{\gamma_{1}, \gamma_{2}, \gamma_{3}\right\}=\varnothing$. Тогда по лемме $1\left\langle\alpha, \gamma_{3}\right\rangle \neq 2,\left\langle\beta, \gamma_{3}\right\rangle \neq 2$, $\left\langle\gamma, \gamma_{3}\right\rangle \neq 2$. Поэтому из равенства $2=\left\langle\gamma_{1}+\gamma_{2}+\gamma_{3}, \gamma_{3}\right\rangle=\left\langle\alpha+\beta+\gamma, \gamma_{3}\right\rangle=\left\langle\alpha, \gamma_{3}\right\rangle+$ $\left\langle\beta, \gamma_{3}\right\rangle+\left\langle\gamma, \gamma_{3}\right\rangle$ следует, что два из чисел Картана $\left\langle\alpha, \gamma_{3}\right\rangle,\left\langle\beta, \gamma_{3}\right\rangle,\left\langle\gamma, \gamma_{3}\right\rangle$ равны 1. Можно считать, что $\left\langle\beta, \gamma_{3}\right\rangle=\left\langle\gamma, \gamma_{3}\right\rangle=1$, a $\left\langle\alpha, \gamma_{3}\right\rangle=0$. При этих условиях $\left[e_{\gamma}, e_{-\gamma_{3}}\right] \neq 0$ (лемма 1$)$ и

$$
\begin{aligned}
0 & =d \psi\left(e_{-\alpha}, e_{-\beta}, e_{-\gamma_{3}}\right)=\left[\psi\left(e_{-\alpha}, e_{-\beta}\right), e_{-\gamma_{3}}\right]+\left[\psi\left(e_{-\alpha}, e_{-\gamma_{3}}\right), e_{-\beta}\right] \\
& =\widehat{\psi}\left(e_{-\alpha}, e_{-\beta}\right)\left[e_{-\gamma}, e_{-\gamma_{3}}\right]+\left[\psi\left(e_{-\alpha}, e_{-\gamma_{3}}\right), e_{-\beta}\right] .
\end{aligned}
$$


Поясним равенство (10). Так как $\langle\alpha, \beta\rangle=0,\left\langle\alpha, \gamma_{3}\right\rangle=0,\left\langle\beta, \gamma_{3}\right\rangle=1$, то $\alpha+\beta, \alpha+\gamma_{3}$, $\beta+\gamma_{3}$ не являются корнями и $\left[e_{-\alpha}, e_{-\beta}\right]=0,\left[e_{-\alpha}, e_{-\gamma_{3}}\right]=0,\left[e_{-\beta}, e_{-\gamma_{3}}\right]=0$. Далее, $\psi\left(e_{-\beta}, e_{-\gamma_{3}}\right)$ отлично от нуля, только если $\lambda=-\beta-\gamma_{3}+\mu$ является корнем. Но $\lambda=-\beta-\gamma_{3}+\alpha+\beta+\gamma=-\gamma_{3}+\alpha+\gamma=\left(\gamma-\gamma_{3}\right)+\alpha$. Так как $\left\langle\gamma, \gamma_{3}\right\rangle=1$, то $\gamma-\gamma_{3} \in R$ и $\left\langle\gamma-\gamma_{3}, \alpha\right\rangle=\langle\gamma, \alpha\rangle-\left\langle\gamma_{3}, \alpha\right\rangle=0$. Следовательно, $\left(\gamma-\gamma_{3}\right)+\alpha=\lambda$ не является корнем и $\psi\left(e_{-\beta}, e_{-\gamma_{3}}\right)=0$.

Таккак $\left[e_{\gamma}, e_{-\gamma_{3}}\right] \neq 0$ и $\sigma=-\alpha-\gamma_{3}+\mu \in R\left(\sigma=-\alpha-\gamma_{3}+\alpha+\beta+\gamma=\left(\gamma-\gamma_{3}\right)+\beta\right.$ и $\left.\left\langle\gamma-\gamma_{3}, \beta\right\rangle=\langle\gamma, \beta\rangle-\left\langle\gamma_{3}, \beta\right\rangle=0-1=-1\right)$, то из равенства (10) получаем, что $\widehat{\psi}\left(e_{-\alpha}, e_{-\beta}\right)=\widehat{\psi}\left(e_{-\alpha}, e_{-\gamma_{3}}\right)$. Так как $\mu=\alpha+\gamma_{3}+\sigma$ и $\left\{\alpha, \sigma, \gamma_{3}\right\} \cap\left\{\gamma_{1}, \gamma_{2}, \gamma_{3}\right\} \neq \varnothing$, то $\widehat{\psi}\left(e_{-\alpha}, e_{-\gamma_{3}}\right)=\widehat{\psi}\left(e_{-\gamma_{1}}, e_{-\gamma_{2}}\right)$.

Таким образом, $\widehat{\psi}\left(e_{-\gamma_{1}}, e_{-\gamma_{2}}\right)=\widehat{\psi}\left(e_{-\alpha}, e_{-\beta}\right)$ для любых $\gamma_{1}, \gamma_{2} \in R$ таких, что $-\gamma_{1}-\gamma_{2}+\mu \in R$. Следовательно, $\operatorname{dim} Z_{\mu}^{2}(L, L) \leqslant 1$.

Покажем, что $\psi_{0}=\sum_{-\gamma_{1}-\gamma_{2}+\mu \in R} e_{-\gamma_{1}}^{*} \wedge e_{-\gamma_{2}}^{*} \otimes e_{-\gamma_{1}-\gamma_{2}+\mu}$ является коциклом.

Пусть $\delta_{1}, \delta_{2}, \delta_{3} \in R$. Если $-\delta_{1}-\delta_{2}-\delta_{3}+\mu \notin R \cup\{0\}$, то $d \psi_{0}\left(e_{-\delta_{1}}, e_{-\delta_{2}}, e_{-\delta_{3}}\right)=0$.

Если $-\delta_{1}-\delta_{2}-\delta_{3}+\mu=0$, то из (9) следует, что $d \psi_{0}\left(e_{-\delta_{1}}, e_{-\delta_{2}}, e_{-\delta_{3}}\right)=h_{\delta_{3}}+$ $h_{\delta_{2}}+h_{\delta_{1}}=0$ (так как $\mu \in \Lambda_{2}(R)$ и, следовательно, $h_{\delta_{3}}+h_{\delta_{2}}+h_{\delta_{1}}$ в алгебрах Ли $A_{5}, D_{6}, E_{7}$ принадлежит центру).

Пусть $\delta=-\delta_{1}-\delta_{2}-\delta_{3}+\mu \in R$. Предположения $\delta_{1}=-\delta_{2}$ или $\delta_{1}=-\delta$ приводят к представлению $\mu$ в виде суммы двух корней, что противоречит утверждению (6) предложения 7 . Если $\delta_{1}=\delta_{2}$, то $d \psi_{0}\left(e_{-\delta_{1}}, e_{-\delta_{2}}, e_{-\delta_{3}}\right)=0$. Следовательно, можем предполагать, что $\delta_{1} \neq \pm \delta_{2}, \delta \neq-\delta_{1}$. Аналогично, $\delta_{1} \neq \pm \delta_{3}, \delta_{2} \neq \pm \delta_{3}, \delta \neq-\delta_{2}$, $\delta \neq-\delta_{3}$.

Поскольку $-\delta_{1}-\delta_{2}-\delta_{3}+\mu \in R$, то слагаемые

$$
\psi_{0}\left(\left[e_{-\delta_{1}}, e_{-\delta_{2}}\right], e_{-\delta_{3}}\right) \text { и }\left[\psi_{0}\left(e_{-\delta_{1}}, e_{-\delta_{2}}\right), e_{-\delta_{3}}\right]
$$

отличны от нуля тогда и только тогда, когда $\delta_{1}+\delta_{2} \in R,-\delta_{1}-\delta_{2}+\mu=$ $\delta_{3}+\delta \in R$ соответственно. Аналогично, равенство нулю остальных слагаемых в $d \psi_{0}\left(e_{-\delta_{1}}, e_{-\delta_{2}}, e_{-\delta_{3}}\right)$ зависит от того, являются $\delta_{2}+\delta_{3}, \delta_{1}+\delta_{3}, \delta_{1}+\delta, \delta_{2}+\delta$ корнями или нет. Поэтому рассмотрим множество $Q=\left\{\delta_{1}+\delta_{2}, \delta_{2}+\delta_{3}, \delta_{1}+\delta_{3}, \delta_{1}+\delta\right.$, $\left.\delta_{2}+\delta, \delta_{3}+\delta\right\}$. Так как по определению $\psi_{0}$ любое слагаемое в $d \psi_{0}\left(e_{-\delta_{1}}, e_{-} \delta_{2}, e_{-\delta_{3}}\right)$ либо равно нулю, либо равно $e_{\delta}$, то $d \psi_{0}\left(e_{-} \delta_{1}, e_{-} \delta_{2}, e_{-\delta_{3}}\right)=0$ в том и только том случае, когда $R \cap Q$ содержит четное количество элементов.

Если $Q \cap R=\varnothing$, то $d \psi_{0}\left(e_{-\delta_{1}}, e_{-\delta_{2}}, e_{-\delta_{3}}\right)=0$. Предположим, что $Q \cap R \neq \varnothing$ и, например, $\delta_{1}+\delta_{2} \in R\left(\left\langle\delta_{1}, \delta_{2}\right\rangle=-1\right)$. Так как $\mu=\left(\delta_{1}+\delta_{2}\right)+\delta_{3}+\delta$, то $\left\langle\delta, \delta_{1}+\delta_{2}\right\rangle=\left\langle\delta, \delta_{3}\right\rangle=\left\langle\delta_{1}+\delta_{2}, \delta_{3}\right\rangle=0$ (в начале доказательства показано, что для любого представления $\mu$ в виде суммы трех корней числа Картана этих корней равны 0$)$. Из условий $\delta \neq-\delta_{1}, \delta \neq-\delta_{2},\left\langle\delta, \delta_{1}+\delta_{2}\right\rangle=0$ и леммы 1 следует, что $\delta \neq \pm \delta_{1}, \delta \neq \pm \delta_{2}$.

Поскольку $\mu \in \Lambda_{2}(R)$, то $\left\langle\mu, \delta_{1}\right\rangle=\left\langle\delta_{1}+\delta_{2}, \delta_{1}\right\rangle+\left\langle\delta_{3}, \delta_{1}\right\rangle+\left\langle\delta, \delta_{1}\right\rangle=1+$ $\left\langle\delta_{3}, \delta_{1}\right\rangle+\left\langle\delta, \delta_{1}\right\rangle \equiv 0(2)$ и $\left\langle\mu, \delta_{2}\right\rangle=\left\langle\delta_{1}+\delta_{2}, \delta_{2}\right\rangle+\left\langle\delta_{3}, \delta_{2}\right\rangle+\left\langle\delta, \delta_{2}\right\rangle=1+\left\langle\delta_{3}, \delta_{2}\right\rangle+$ $\left\langle\delta, \delta_{2}\right\rangle \equiv 0(2)$. Отсюда следует, что $\left\langle\delta_{3}, \delta_{1}\right\rangle+\left\langle\delta, \delta_{1}\right\rangle= \pm 1$ и $\left\langle\delta_{3}, \delta_{2}\right\rangle+\left\langle\delta, \delta_{2}\right\rangle= \pm 1$ (так как $\delta_{3} \neq \pm \delta_{1}, \delta_{3} \neq \pm \delta_{2}, \delta \neq \pm \delta_{1}, \delta \neq \pm \delta_{2}$ ). Так как $\left\langle\delta, \delta_{1}+\delta_{2}\right\rangle=\left\langle\delta_{1}+\right.$ $\left.\delta_{2}, \delta_{3}\right\rangle=0$, то, не ограничивая общности, можем считать, что $\left\langle\delta_{3}, \delta_{1}\right\rangle+\left\langle\delta, \delta_{1}\right\rangle=1$ и $\left\langle\delta_{3}, \delta_{2}\right\rangle+\left\langle\delta, \delta_{2}\right\rangle=-1$. Из этих равенств при условиях $\delta_{3} \neq \pm \delta_{1}, \delta_{3} \neq \pm \delta_{2}$, $\delta \neq \pm \delta_{1}, \delta \neq \pm \delta_{2}$ получаем, что только одно из чисел $\left\langle\delta_{3}, \delta_{1}\right\rangle,\left\langle\delta, \delta_{1}\right\rangle,\left\langle\delta_{3}, \delta_{2}\right\rangle,\left\langle\delta, \delta_{2}\right\rangle$ 
равно -1 . Тогда из леммы 1 следует, что корнем является единственный элемент из множества $\left\{\delta_{2}+\delta_{3}, \delta_{1}+\delta_{3}, \delta_{1}+\delta, \delta_{2}+\delta\right\}$.

Если предположить, что $\delta_{3}+\delta \in R$, то получим представление $\mu=\left(\delta_{1}+\delta_{2}\right)+$ $\left(\delta_{3}+\delta\right)$, что противоречит утверждению (6) предложения 7. Следовательно, $\delta_{3}+\delta \notin R$.

Таким образом, из предположения $\delta_{1}+\delta_{2} \in R$ следует, что ровно два элемента из множества $Q$ являются корнями, а следовательно, ровно два слагаемых в $d \psi_{0}\left(e_{-\delta_{1}}, e_{-\delta_{2}}, e_{-\delta_{3}}\right)$ отличны от нуля и $d \psi_{0}\left(e_{-\delta_{1}}, e_{-\delta_{2}}, e_{-\delta_{3}}\right)=0$. Аналогично, $d \psi_{0}\left(e_{-\delta_{1}}, e_{-\delta_{2}}, e_{-\delta_{3}}\right)=0$, если корнем является любой другой элемент из $Q$.

Поэтому $\psi_{0}$ является коциклом и, следовательно, $\operatorname{dim} Z_{\mu}^{2}(L, L)=1$. Так как $\operatorname{dim} B_{\mu}^{2}(L, L)=0$, то $\operatorname{dim} H_{\mu}^{2}(L, L)=1$ и утверждение $(2)$ доказано.

Теперь мы можем привести доказательство основной теоремы, сформулированной во введении.

Если $L$ имеет тип $A_{2}$, то $H^{2}(L, L)=0$ согласно предложению 2. Если $L$ имеет тип $A_{l}, l \neq 2,3,5, \bar{A}_{l}, l \neq 3,5, l \equiv 1(2), E_{6}, E_{8}$, то из результатов $\S 3$ и предложений 7,8 следует, что $H^{2}(L, L)=0$. Если $L$ имеет тип $A_{3}$ или $D_{l}, l \equiv 1(2)$, то $H^{2}(L, L)=0$ согласно результатам $\S 3$ и предложениям $7-9,11$. Если $L$ имеет тип $A_{5}$ или $E_{7}$, то $H^{2}(L, L)=0$ согласно результатам $\S 3$ и предложениям $7,8,13$. Если $L$ имеет тип $\bar{D}_{l}, l \equiv 0(2), l \neq 6$, то $H^{2}(L, L)=0$ согласно результатам $\S 3$ и предложениям 7-9, 12 .

Таким образом, утверждение (1) основной теоремы доказано.

Если $L$ имеет тип $\bar{A}_{3}$, то $\operatorname{dim} H_{2}(L, L)=20$ согласно теореме 2 . Если $L$ имеет тип $\overline{A_{5}}$, то из результатов $\S 3$ и предложений $7,8,13$ следует, что $H^{2}(L, L)=$ $\bigoplus_{\mu \in M} H_{\mu}^{2}(L, L)$, где $M=\{\alpha+\beta+\gamma \mid\langle\alpha, \beta\rangle=\langle\alpha, \gamma\rangle=\langle\beta, \gamma\rangle=1\}$. Согласно лемме $14|M|=20$. Тем самым, утверждение (2) основной теоремы доказано.

Если $L$ имеет тип $D_{4}$, то из результатов $\S 3$ и предложений 7-9, 11 следует, что $H^{2}(L, L)=\bigoplus_{\mu \in M} H_{\mu}^{2}(L, L)$, где $M=\{\gamma+\delta \mid\langle\gamma, \delta\rangle=0\}$. Согласно лемме 13 $|M|=64$, поэтому выполняется утверждение (3) основной теоремы.

Если $L$ имеет тип $\bar{D}_{l}, l \equiv 1(2)$, то $\operatorname{dim} H^{2}(L, L)=2 l$ согласно теореме 1 . Если $L$ имеет тип $D_{l}, l \equiv 0(2), l>6$, то из результатов $\S 3$ и предложений $7-9,11$ следует, что $H^{2}(L, L)=\bigoplus_{\mu \in M} H_{\mu}^{2}(L, L)$, где $M=\{\gamma+\delta \mid\langle\gamma, \delta\rangle=0\}$. Согласно лемме 13 $|M|=2 l$, поэтому $\operatorname{dim} H_{2}(L, L)=2 l$. Если $L$ имеет тип $D_{6}$, то $\operatorname{dim} H^{2}(L, L)=12$ согласно $\S 3$ и предложениям $7-9,11,13$. Тем самым, утверждение $(4)$ основной теоремы доказано.

Если $L$ имеет тип $\bar{D}_{6}$, то согласно $\S 3$ и предложениям $7-9,12,13 H^{2}(L, L)=$ $\bigoplus_{\mu \in M} H_{\mu}^{2}(L, L)$, где $M=\{\alpha+\beta+\gamma \mid\langle\alpha, \beta\rangle=\langle\alpha, \gamma\rangle=\langle\beta, \gamma\rangle=1\}$. Согласно лемме $14|M|=64$. Если $L$ имеет тип $\bar{E}_{7}$, то из результатов $\S 3$ и предложений 7 , 8,13 следует, что $H^{2}(L, L)=\bigoplus_{\mu \in M} H_{\mu}^{2}(L, L)$, где $M=\{\alpha+\beta+\gamma \mid\langle\alpha, \beta\rangle=$ $\langle\alpha, \gamma\rangle=\langle\beta, \gamma\rangle=1\}$. Согласно лемме $14|M|=56$. Следовательно, вьполняются утверждения (5) и (6) основной теоремы.

\section{Список литературы}

1. Кострикин А. И. Параметрическое семейство простых алгебр Ли // Изв. АН СССР. Сер. матем. 1970. Т. 34. С. $744-756$.

2. Джумадильдаев А. С. К деформациям классических простых алгебр Ли // УМН. 1976. Т. 31. № 3(189). С. 211-212. 
3. Кострикин А.И., Кузнецов М.И. О деформациях классических алгебр Ли характеристики три // Докл. РАН. 1995. Т. 343. № 3. С. 299-301.

4. Рудаков А. Н. Деформации простых алгебр Ли // Изв. АН СССР. Сер. матем. 1971. T. 35. С. $1113-1119$.

5. Кузнецов М.И., Чебочко Н.Г. Деформации классических алгебр Ли // Матем. сб. 2000. T. 191. № 8. С. $69-88$.

6. Кириллов С. А., Кузнецов М. И., Чебочко Н. Г. О деформациях алгебры Ли типа $G_{2}$ характеристики три // Изв. вузов. Сер. матем. 2000. №3 (454). С. 33-38.

7. Shen Guangyu Variations of the classical Lie algebra $G_{2}$ in low characteristics // Nova J. Alg. Geom. 1993. V. 2. № 3. P. 217-243.

8. Frohardt D. E., Griess R. L., Jr. Automorphisms of modular Lie algebras // Nova J. Alg. Geom. 1992. V. 1. № 4. P. 339-345.

9. Бурбаки Н. Группы и алгебры Ли. Гл. IV-VI. М.: Мир, 1972.

10. Стейнберг Р. Лекции о группах Шевалле. М.: Мир, 1975.

11. Гишарде A. Когомологии топологических групп и алгебр Ли. М.: Мир, 1984.

12. Бурбаки Н. Группы и алгебры Ли. Гл. VII-VIII. М.: Мир, 1978.

13. Кострикин А.И., Шафаревич И. Р. Градуированные алгебры Ли конечной характеристики // Изв. АН СССР. Сер. матем. 1969. Т. 33. С. 251-322.

Нижегородский государственный университет

Поступила в редакцию им. Н.И. Лобачевского

29.11.2004

E-mail: kuznets@unn.ac.ru 